KBC-40179

\title{
ALARA Assessment of Settler Sludge Sampling Methods
}

Prepared for the U.S. Department of Energy

Assistant Secretary for Environmental Management

Contractor for the U.S. Department of Energy

under Contract DE-AC06-08RL14788

CH2MHILL

Plateau Remediation Company

P.O. Box 1600

Richland, Washington 99352 
KBC-40179

Revision 1

EDC \#: HNF-EDC-09-40324

\section{ALARA Assessment of Settler Sludge Sampling Methods}

Document Type: DD

Program/Project: STP

L. A. Nelsen

CH2M HILL Plateau Remediation Company

Date Published

March 2009

Prepared for the U.S. Department of Energy

Assistant Secretary for Environmental Management

Contractor for the U.S. Department of Energy

under Contract DE-AC06-08RL14788

\section{CH2MHILL}

Plateau Remediation Company

P.O. Box 1600

Richland, Washington
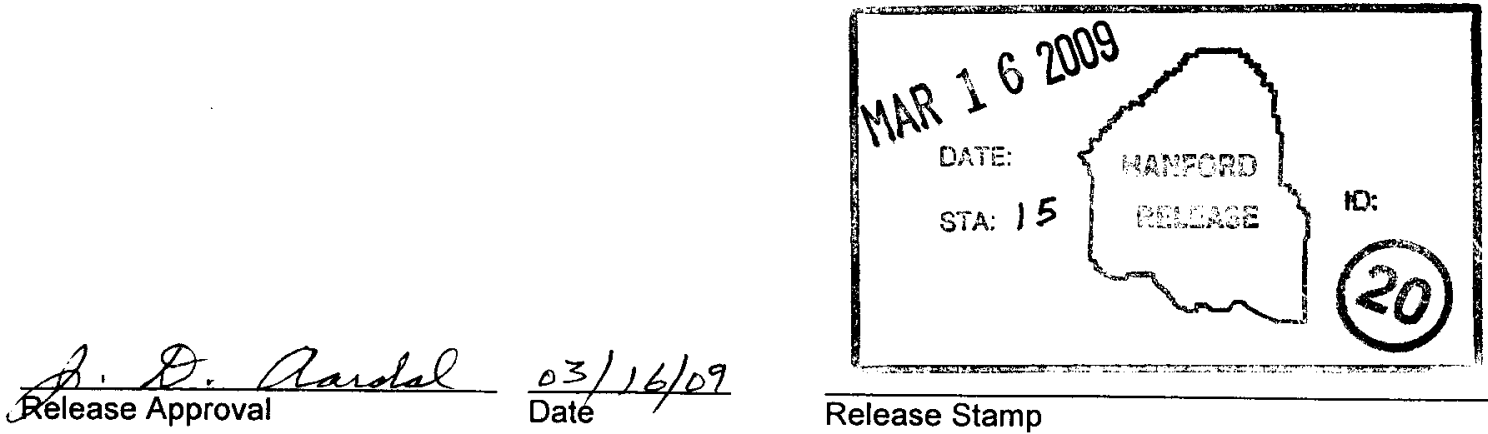

Release Stamp

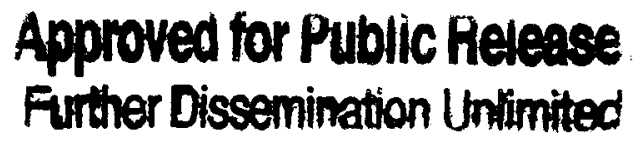




\section{TRADEMARK DISCLAIMER}

Reference herein to any specific commercial product, process,

or service by trade name, trademark, manufacturer, or otherwise, does not necessarily constitute or imply its

endorsement, recommendation, or favoring by the United

States Government or any agency thereof or its contractors or subcontractors.

This report has been reproduced from the best available copy.

Printed in the United States of America

Total Pages: $\quad 36$ 


\section{RECORD OF REVISION}

(2) Title

ALARA Assessment of Settler Sludge Sampling Methods

\section{Change Control Record}

(3)

Revision

0

(4) Description of Change - Replace, Add, and Delete Pages

Intial Revision - EDC \#HNF-EDC-09-40288

RS

Revision to document to revise Dollar savings, to correct

pages changed. EDC \#HNF-EDC-09-40324
Authorized for Release

(5) DATTA

Date

LA Nelsen

01/30/2009

LA Nelsen

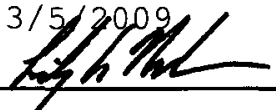




\section{Purpose:}

The purpose of this assessment is to compare underwater and above water settler sludge sampling methods to determine if the added cost for underwater sampling for the sole purpose of worker dose reductions is justified. Initial planning for sludge sampling included container, settler and knock-out-pot (KOP) sampling. Due to the significantly higher dose consequence of KOP sludge, a decision was made to sample KOP underwater to achieve worker dose reductions. Based on the perception that doses would be prohibitive for settler sludge also, initial plans were to utilize a similar underwater sampling apparatus for the settler sludge. Since there are no longer plans to sample KOP sludge, the decision for underwater sampling for settler sludge needs to be revisited. The present sampling plan calls for spending an estimated $\$ 1,977,000$ to design and construct a new underwater sampling system, these costs are shown in Table 9 of this document. This evaluation will compare and contrast the present method of above water sampling to the underwater method that is planned by the Sludge Treatment Project (STP) and determine if settler samples can be taken using the existing sampling cart (with potentially minor modifications) while maintaining doses to workers As Low As Reasonably Achievable (ALARA) and eliminate the need for costly redesign, testing, personnel retraining and new operating procedures.

\section{Background:}

The sludge material has been collected from various areas in the $\mathrm{KE}$ and $\mathrm{KW}$ basins and is being stored in the engineered containers (SCS-CON-210,-220,-240,-250 and -260). The project requires that samples be taken of the sludge material so that appropriate safety and operational features can be built into the transportation, storage and processing equipment to verify that the waste forms will meet the various state and federal requirements.

The present location of the six sludge containers is in the center and east bay of the $\mathrm{K}$ West Basin. Three containers (SCS-CON-240, -250 , and -260) are filled with sludge that originated in the K East Basin. Two containers (SCS-CON-210 and -220) are filled with sludge from $\mathrm{K}$ West Basin. The remaining container (SCS-CON-230, presently empty) will be used for sludge that is scheduled to be transferred from the 10 settler tanks. The material that will be transferred to SCS-CON-230 is referred to as "settler" sludge, and the material from the other 5 containers (SCS-CON-210, $-220,-240,-250$, and -260 ) is referred to as "container" sludge.

Prior to the collection of settler sludge samples from SCS-CON-230, container sludge samples will be taken from SCS-CON-210, $-220,-240,-250$, and -260 . The method scheduled to be used to conduct sampling of these first 5 containers is an above water sampling cart which is very similar to the equipment that has been used during previous sampling campaigns (see Figure 1).

The next phase of the sludge sampling will entail the sampling of the settler sludge which will have been transferred into SCS-CON-230. The method presently planned to complete settler sampling (see figure 2) differs from the container sampling method in the following ways:

- Sample bottles will be filled underwater in the basin.

- The guide, isolation, and extraction tubes will change to allow the sample material to be diverted to the sample bottles while maintaining a water cover (see Figure 3 for representation of container with isolation and guide tubes)

- A larger sample bottle set will be used so that bottles do not have to be changed during the sampling of one location

- A shielded shipping container (pig) with additional shielding is planned due to the anticipated radiological content of the sludge 
The sludge that will be transferred into engineered container SCS-CON-230 is still located in the settler tanks (See Figure 4 for view of settler tanks). The material from the settler tanks is anticipated to have higher curie content and be denser than sludge that is in the remaining containers. Once the settler contents have been relocated into the engineered container, sampling will be performed. The planned sample method will use an underwater method to provide the required shielding and maintain worker dose ALARA. The resulting sample bottles will be transferred from KW Basin to the analytical laboratory using a PAS-1 cask.

While the settler sludge sampling campaign has not yet been designed, the sampling process for the remaining container sludge (SCS-CON-210,-220,-240,-250 and -260) is well understood and employs a system for above water sampling that has been successfully employed during previous sludge sampling campaigns at the K-basins (see Figure 1). Some of the ALARA features incorporated into design and operations of the container sludge sampling system include the following (these items will also be applicable to above water settler sludge sampling):

- The use of the MASF Building for mockup training.

- In Basin (dry run) Testing.

- Shielded sample cart pigs to house sample bottles, while in cart

- HEPA filters used on pump outlet and vacuum breaker vents.

- Vacuum system that precludes spray leaks during sampling and a sample cart that contains any spill that maybe encountered during sampling.

- Radiological controls have been placed into the operating procedure that include dose limits, backflushing of lines, and provisions for handling sample bottles with excessive dose rates.

- Radiological survey requirements have been incorporated in the procedure when there is a potential for individuals to encounter dose rates $\geq 100 \mathrm{mrem} / \mathrm{hr}$. In addition, radiological action limits have been incorporated into the procedure to reduce the chance of exceeding RWP void limits.

- An engineered frame is available to cover the above water sludge sampling line with shielding.

- Handling of filled sample bottles will be minimized (no decanting of samples). A T-bar will be used to maximize the distance between the worker and the filled sample bottles, when transferring bottles from the sampling cart. 
Figure 1 - STP Container Sludge Sampling Cart (Above Water Sampling)

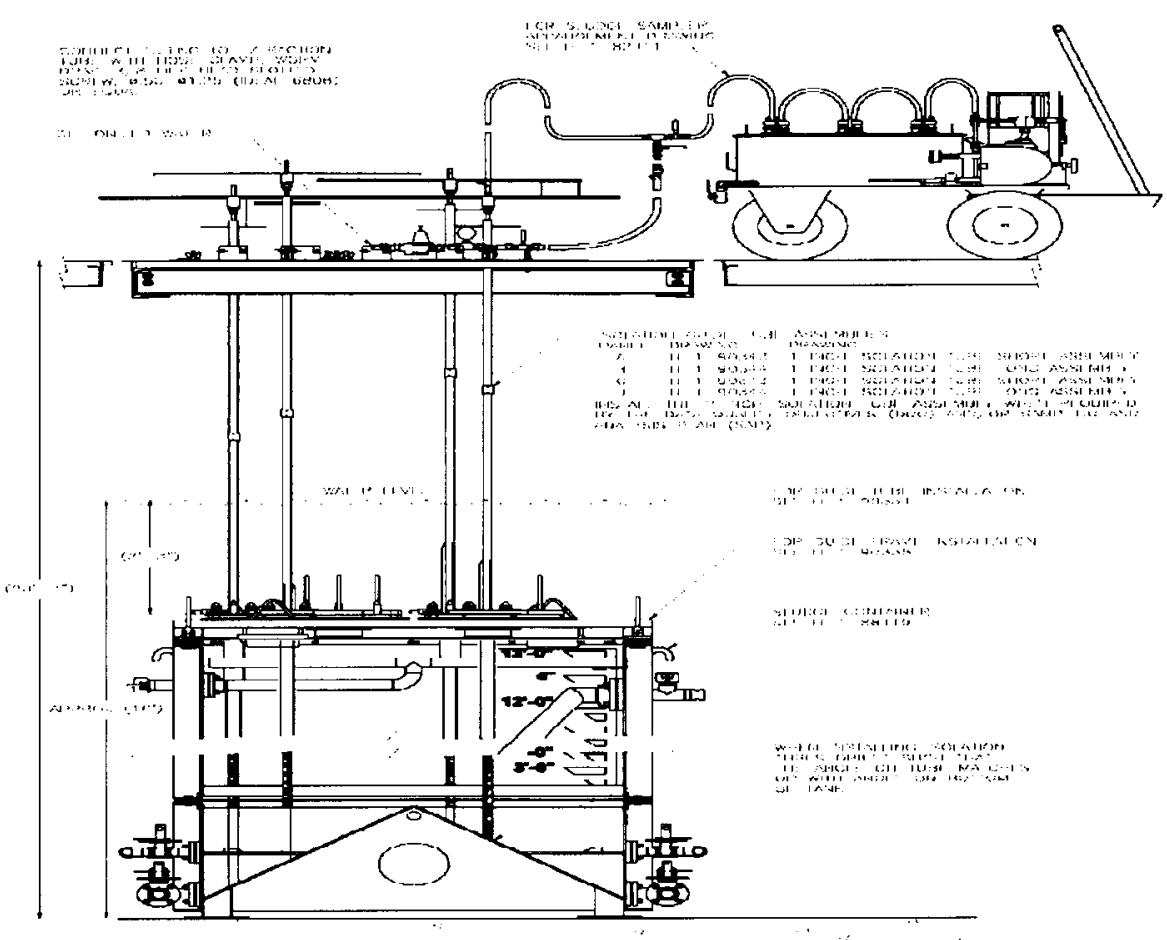

Figure 2 - Present STP "Proposed" Settler Sludge Sampling Method (Underwater)

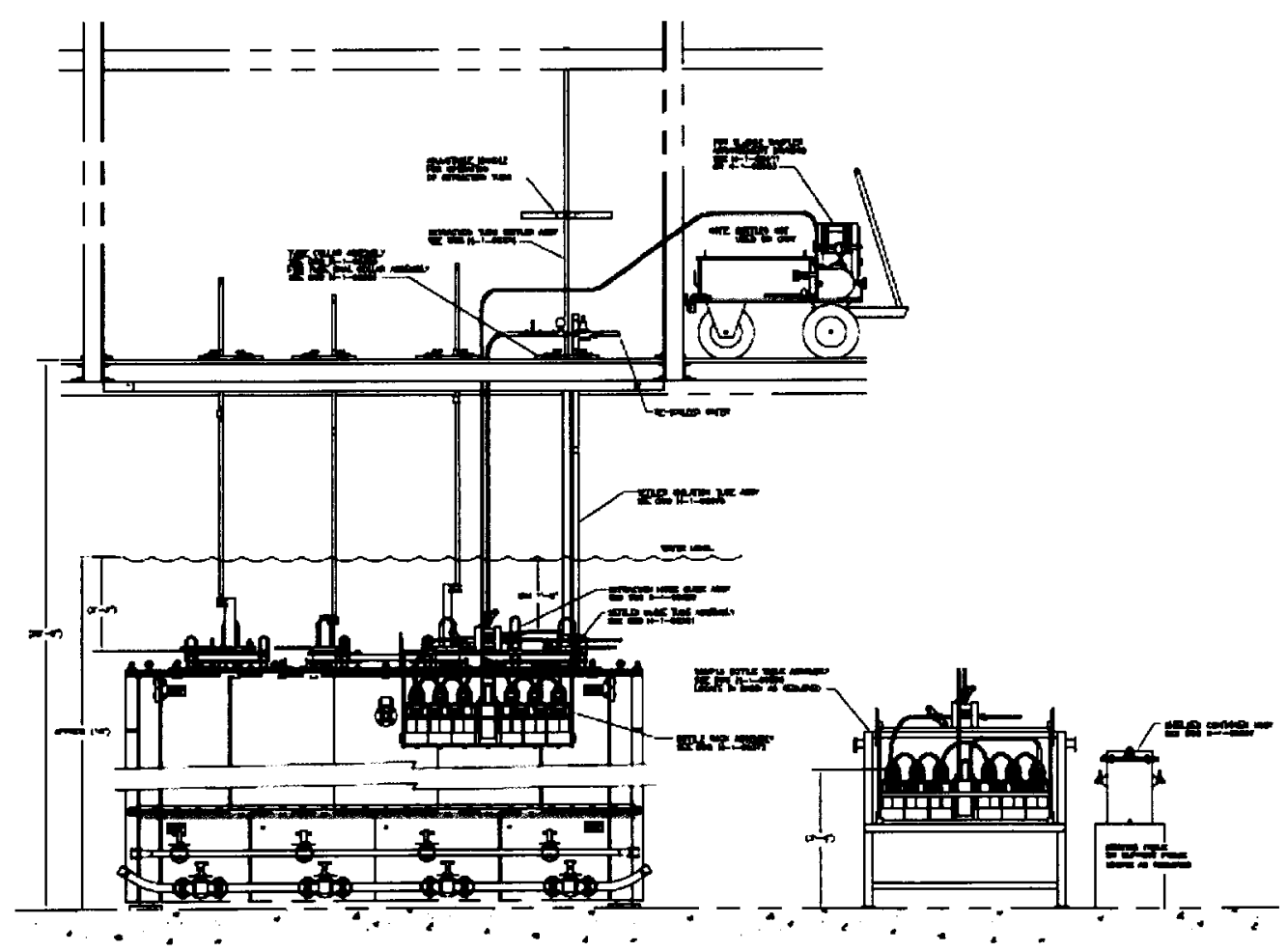


Figure 3 - Grating Level Work Platforms with isolation tubes in place

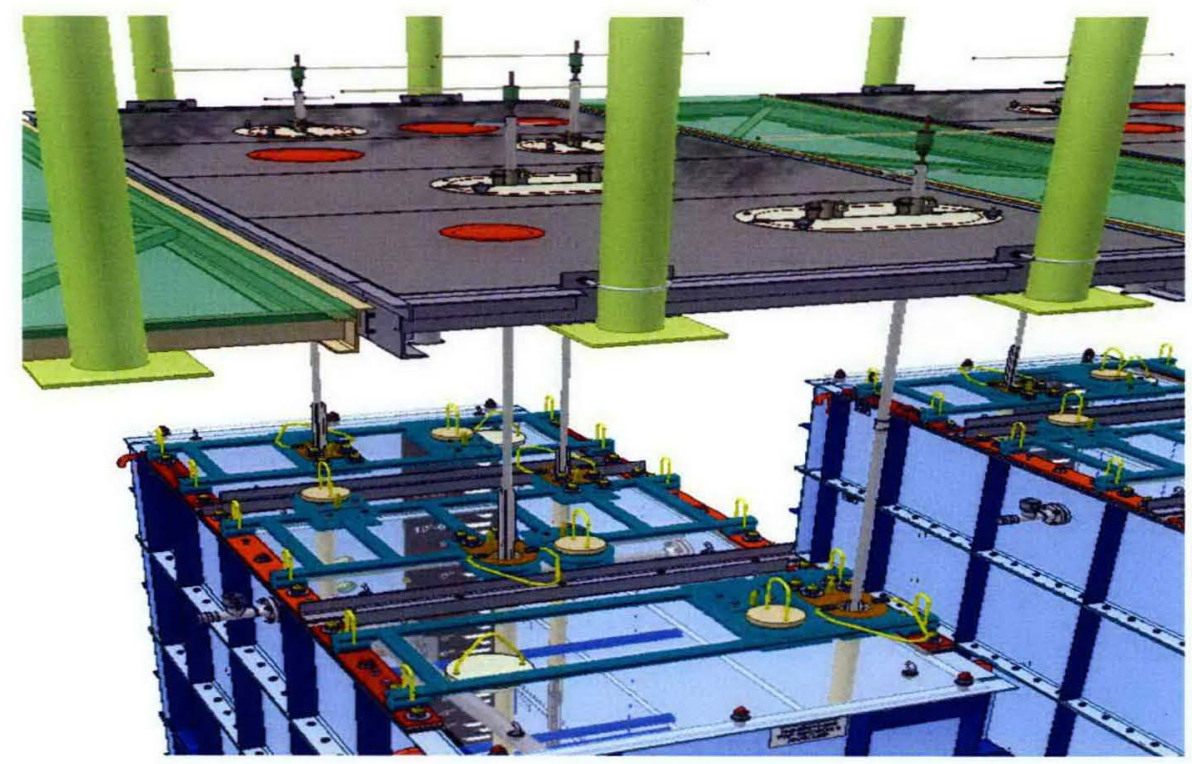

Figure 4 - Settler Tanks

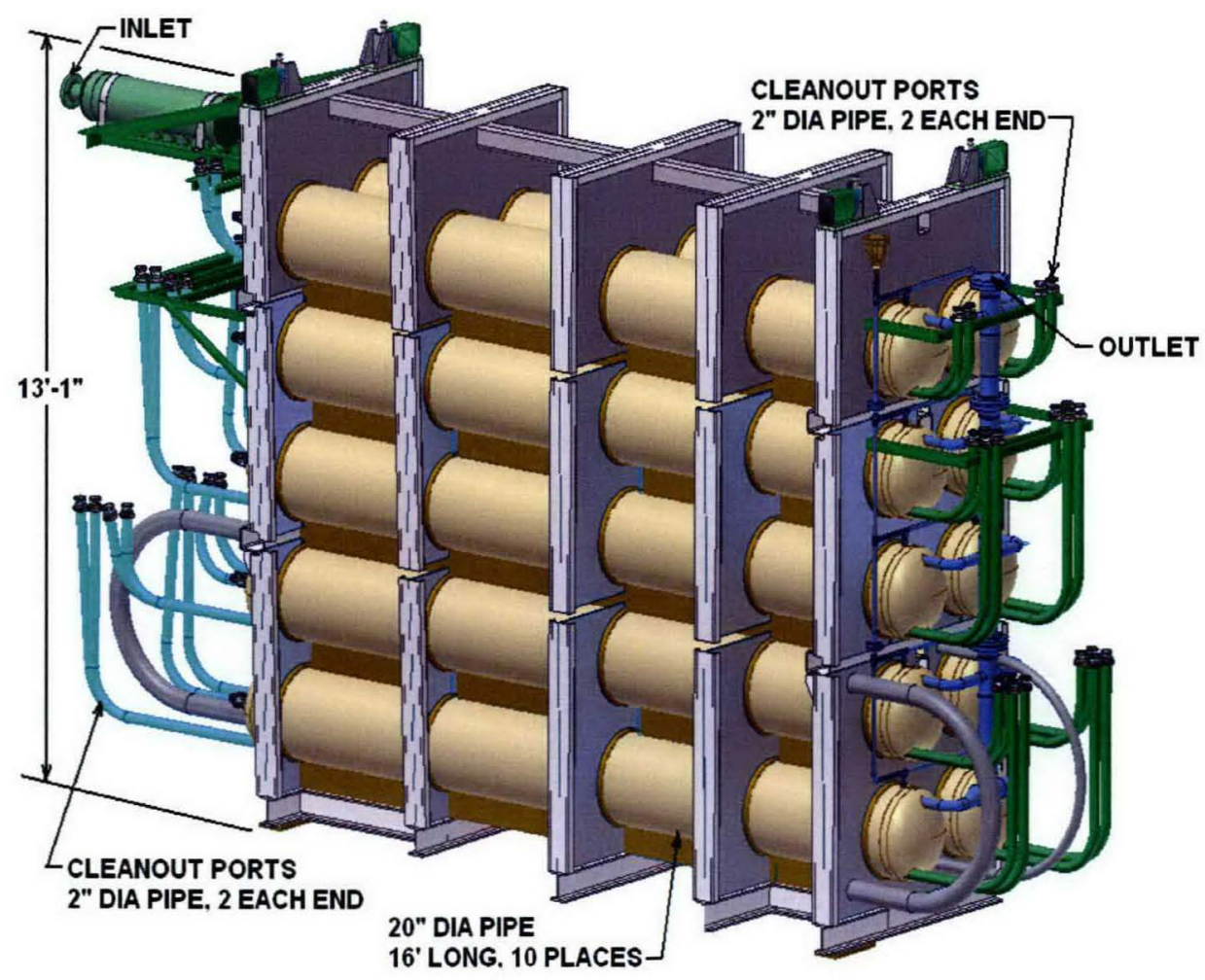




\section{Assessment:}

Calculations completed during this assessment were performed using a Dell Optiplex 755, Intel Core 2 Duo processor, CHPRC Computer \#WC95262. The calculations were performed using site wide licensed Microshield, version 7.02, issued under document \#CHPRC-00064, The proper installation and functionality of Microshield, Version 7.02 on CHPRC computer \#WC95262 has been documented in the STP Radiological Engineers field logbook by successful completion of the installed Verification and Validation, results are attached to revision 0 of KBC-40179.

This assessment performs a number of calculations to evaluate dose rates for settler sludge during various sampling activities. The methods used in this calculation are the same as were used in ALARA report KBC-37814 to determine dose rates for container sludge sampling. The dose rates calculated using methods outlined in the ALARA report were then used to determine expected person-rem doses for the settler sludge sampling evolution. This same process is outlined in Letter \#09-100K-GLH-003 for container sludge. The processes outlined in these two documents were used for this assessment to determine the dose that can be anticipated if settler samples are taken above water using existing sampling equipment.

The following assumptions were used throughout this assessment and for the calculations associated with this assessment.

Assumptions:

- All samples were assumed to be based on $400 \mathrm{ml}$ of sludge contained in the $4 \mathrm{~L}$ Nalgene bottle with 2.6 liters of basin water. The number of sample bottles per core sample will vary depending on sludge depth in the container. A total of 4 core samples will be taken with each core assumed to not exceed 1 liter, for a total of 4 liters of sludge, at a maximum of $400 \mathrm{ml}$ per bottle, it is therefore assumed 10 bottles will be necessary. The $400 \mathrm{ml}$ is based on past sampling campaign. Since this analysis assumes a constant concentration for the sludge, varying the number of samples has little affect on the total dose received during the sampling and any difference would be bounded by the contingency already in place in this assessment.

- Source terms for settler sludge and container sludge is as described in HNF-SD-SNF-TI-015 Rev.14, Spent Nuclear Fuel Project Technical Data Book, Vol. 2, Sludge, Table 4-21 and 5-3. The resulting values have been condensed in Figure 5.0 below.

- Standard densities were used for common items: water $1.0 \mathrm{gm} / \mathrm{cm}^{3}$, steel $7.86 \mathrm{gm} / \mathrm{cm}^{3}$, air 0.00122 $\mathrm{gm} / \mathrm{cm}^{3}$

- Settler tanks dimensions: 20 in diameter; 16 feet long; walls are schedule 10 pipe $(0.25$ inch thickness).

- The settler tanks are assumed to be $54 \%$ full, per the HNF-SD-SNF-TI-015 data book.

- Settler tanks contain $5.4 \mathrm{~m}^{3}$ of sludge. \%volume $=5.4 \mathrm{~m}^{3} / \mathrm{V}$ (volume of single settler tank) where $=V=\pi r^{2} \mathrm{~h}$

- Settler tank dose measurements taken per work package 1K-07-03241 used an RO-07 with distance to center of detector of 1 inch. The 1 inch will be used as the "Contact" distance for any Microshield measurements.

- A Dose Decision Value for Optimization of $\$ 25,000 /$ person-rem was used.

- $\mathrm{KW}$ basin general area background dose rate assumes $\sim 1 \mathrm{mr} / \mathrm{hr}$.

- The neutron dose rate from this source term is negligible compared to the photon dose rate and was not included (see calculation A21C-N-003).

- One settler sample bottle per PAS-1 Cask shipment. 
- The dimensions for the KW basin sludge sample bottle are Height $54.61 \mathrm{~cm}$ and the OD is 16.193 $\mathrm{cm}$.

- The primary exposure occurs during the sample pull and transferring of samples from the sample cart to the SSC for shipment. Minimal exposure will result from $\mathrm{KW}$ basin background $(\sim 1.0 \mathrm{mrem} / \mathrm{h})$.

Figure 5.0 Sludge Source Term

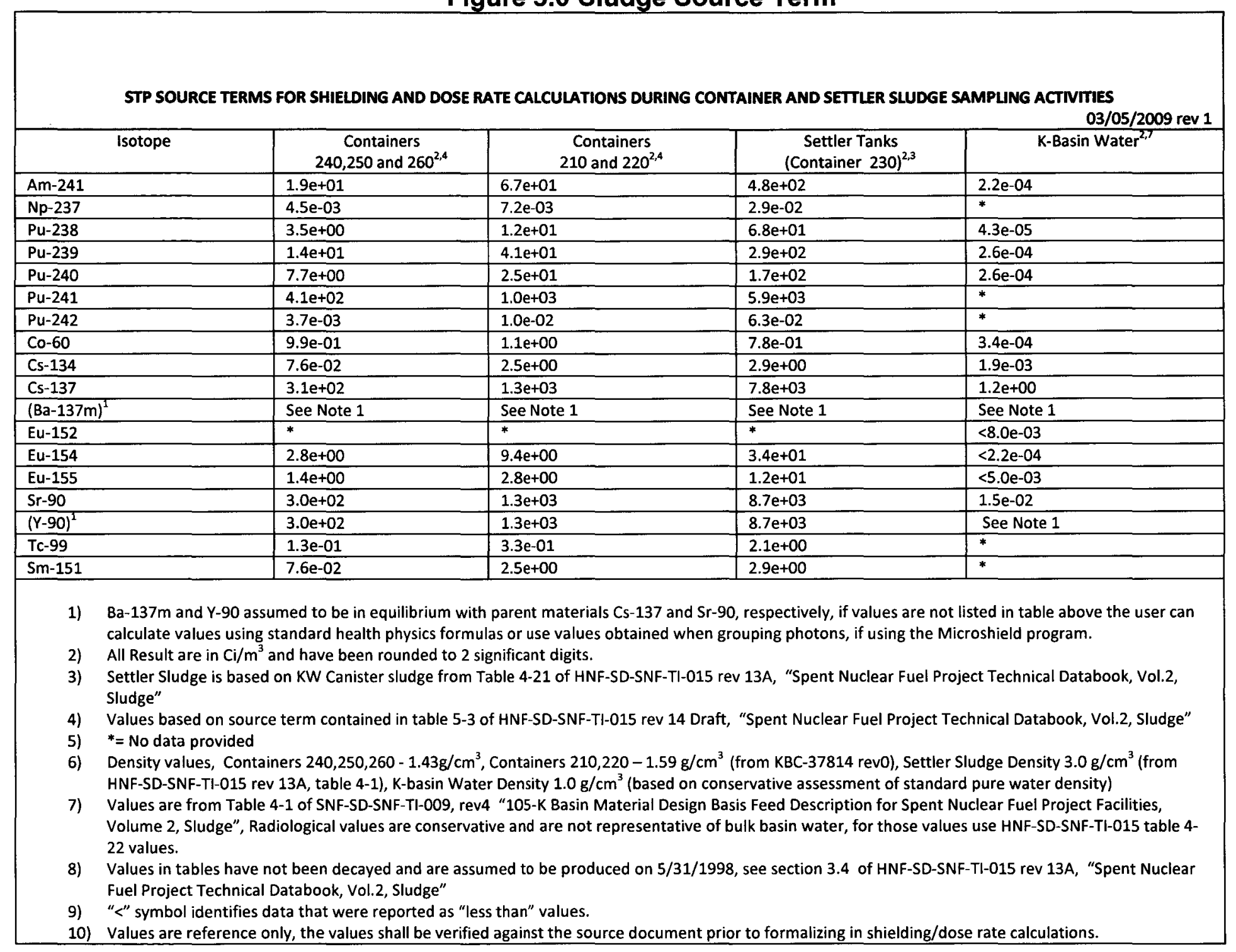

To compare the two calculations it was first necessary to verify that source terms identified in Figure 5 are the same and that the geometry of the source will yield similar results as are found in KBC-37814 (this is especially important in this case since different shielding codes are being employed, Microshield vs.

MCNP). To accomplish this, calculations were conducted assuming an unshielded sample bottle containing $400 \mathrm{ml}$ of settled sludge from each of the three defined source terms (containers 210/220, 240/250/260 and settler sludge, see Microshield runs KBC40179_1,2 and 3). Results from these runs were compared against the results in KBC-37814 and are outlined in Table 1 below: 


\section{Table 1 - Comparison of Unshielded Sample Bottle Dose Rates}

\begin{tabular}{|c|l|l|l|l|}
\hline \multirow{2}{*}{$\begin{array}{c}\text { Results for } \\
\text { KBC-40179_1 }\end{array}$} & \multicolumn{1}{|c|}{$\begin{array}{c}\text { Dose Rate } \\
(\mathrm{mr} / \mathrm{hr})\end{array}$} & Container 210/220 & Container 240/250/260 & Settler Sludge \\
\cline { 2 - 5 } KBC-40179_2 & 1 inch & 18520 & 4541 & 91770 \\
\cline { 2 - 5 } KBC-40179_3 foot & 989.8 & 244.3 & 4569 \\
\cline { 2 - 6 } & 1 meter & 122.6 & 30.3 & 557.7 \\
\cline { 2 - 5 } & 10 feet & 14.5 & 3.58 & N/A \\
\hline $\begin{array}{c}\text { Results from } \\
\text { ALARA Report }\end{array}$ & $1 \mathrm{~cm}^{*}$ & 23491 & 5196 & $\mathrm{~N} / \mathrm{A}$ \\
\cline { 2 - 5 } $\begin{array}{c}\text { KBC-37814 } \\
\text { (Table 15) }\end{array}$ & 1 meter & 1001 & 220.7 & $\mathrm{~N} / \mathrm{A}$ \\
\cline { 2 - 5 } & 10 feet & 141.4 & 30.9 & $\mathrm{~N} / \mathrm{A}$ \\
\hline
\end{tabular}

* MCNP allows for calculation of contact dose rates, the Microshield code is unreliable at distances less than 1 inch. Items N/A'ed were not part of the ALARA report calculations. It should be noted that KBC-37814 document contains an error that was discovered during the review of this document, the calculation incorrectly reports the Ba-137m levels for settler sludge, though it does not affect the results used for this calculation.

A review of the results of Table 1 shows, that the source term and geometry are comparable with results that have been calculated in the sampling of KW sludge containers ALARA report. These results allow it to be said with some certainty, that this is a good model to work from and we can scale results obtained from the ALARA report, when necessary.

With the model validated, the next step was to determine a dose reduction factor (DRF) for the settler tanks, as was completed in Table 14 of KBC-37814. The dose reduction factor is used to relate calculated doses to those that have been found with field deployed survey equipment. The dose reduction factor is determined by calculating the expected dose using the maximum source term for settler sludge, as identified in Table 2, below and modeling it in the configuration of the settler tanks. The dose reduction factor is then the ratio of the highest dose rate calculated to the highest that was obtained using underwater radiation detection equipment. The dose rate results were obtained 08/28/07 under work package $1 \mathrm{~K}-07-03241$ and ranged from 3.2 to $33 \mathrm{R} / \mathrm{hr}$, the calculation will use the $33 \mathrm{R} / \mathrm{hr}$ as this yields the most conservative dose reduction factor.

To complete the calculation to determine the highest calculated dose rate, a Microshield run was completed modeling the settler tanks as follows: 20 in diameter, 16 feet long, with walls of schedule 10 steel ( 0.25 inches). The model was then filled with settler sludge with an assumed maximum activity that was multiplied by $54 \%$ (see assumptions). This is a conservative assessment since the model has more self shielding than would normally be evident and lowers the maximum dose rate obtained, thus reducing the dose reduction factor (see Microshield run KBC40179_4). Table 2 shows the dose reduction factor that was calculated for the settler tanks, the table also lists for comparison, the dose reduction factors calculated in KBC-37418 for the container sludge.

Table 2 Dose Reduction Factor DFR's

\begin{tabular}{|c|c|c|c|}
\hline Container Material & $\begin{array}{c}\text { Calculated Dose Rate, } \\
\text { rem/hr }\end{array}$ & $\begin{array}{c}\text { Maximum measured } \\
\text { dose rate, rem/hr }\end{array}$ & Dose reduction factor \\
\hline Settler Tank & 363.9 & 33 & 11.0 \\
\hline Container 230) & 37.1 & 4 & 9.3 \\
\hline Container 210/220 & 12.5 & 9 & 1.4 \\
\hline
\end{tabular}


The next portion of this assessment deals with calculating various dose rates that can be anticipated from the unshielded sludge sample bottle (USSB) containing settler sludge. This was done by modeling a source in the shape of a Nalgene bottle, that contains $400 \mathrm{ml}$ of settler sludge, and evaluating it at similar distances as were modeled in calculation, KBC-37418 (see Microshield run KBC-40179_3). This same Microshield model was then used in a separate calculation that has the USSB surrounded in lead shielding that simulates the bottle being placed in the sample cart shielded containers (see Microshield run KBC-40179_5). The results are presented in Table 3 below:

Table 3 Container Dose Rates Compared to Settler Dose Rates

\begin{tabular}{|c|c|c|c|c|c|c|}
\hline \multirow[b]{2}{*}{$\begin{array}{l}\text { Source and } \\
\text { dose point } \\
\text { locations }\end{array}$} & \multicolumn{2}{|c|}{ Containers 210 and $220^{\star * * *}$} & \multicolumn{2}{|c|}{ Containers $240 / 250$, and $260^{* * * *}$} & \multicolumn{2}{|c|}{ Settler Sludge (Container 230) } \\
\hline & $\begin{array}{l}\text { Calculated } \\
\text { Dose Rate, } \\
\mathrm{mrem} / \mathrm{h}^{\star * \star}\end{array}$ & $\begin{array}{c}\text { With Dose } \\
\text { Reduction } \\
\text { Factor, } \\
\text { mrem/h }\end{array}$ & 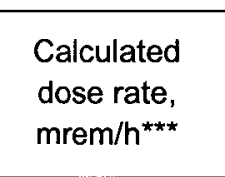 & $\begin{array}{c}\text { With Dose } \\
\text { Reduction } \\
\text { Factor, mrem/h }\end{array}$ & $\begin{array}{l}\text { Calculated } \\
\text { Dose Rate, } \\
\text { mrem/hr*** }\end{array}$ & $\begin{array}{l}\text { With reduction } \\
\text { factor, mrem/h }\end{array}$ \\
\hline \multicolumn{7}{|c|}{ Unshielded Sludge Sample Bottle (USSB) } \\
\hline $1 \mathrm{~cm} / 1 \mathrm{in} *$ & $23,491.0$ & $2,531.2$ & $5,196.5$ & $3,749.3$ & 91770 & 8342.7 \\
\hline $1 \mathrm{ft}$ & $1,001.9$ & 108.0 & 220.7 & 159.2 & 4569 & 415 \\
\hline $1 \mathrm{~m}$ & 141.4 & 15.2 & 30.9 & 22.3 & 557.7 & 50.7 \\
\hline $10 \mathrm{ft}$ & 15.5 & 1.7 & 3.4 & 2.5 & 65.4 & 5.9 \\
\hline \multicolumn{7}{|c|}{ Shielded Sample Bottle in Cart (PIG) } \\
\hline $1 \mathrm{~cm} / 1 \mathrm{in}^{\star}$ & 165.7 & 17.8 & 35.1 & 25.3 & 469.7 & 42.7 \\
\hline $1 \mathrm{ft}$ & 22.3 & 2.4 & 4.7 & 3.4 & 40.4 & 3.7 \\
\hline $1 \mathrm{~m}$ & 1.3 & 0.1 & 0.3 & 0.2 & 5.4 & 0.5 \\
\hline $10 \mathrm{ft}$ & 0.2 & 0.0 & 0.0 & 0.0 & 0.6 & 0 \\
\hline \multicolumn{7}{|c|}{ Unshielded Sludge Sample Hose (USSL) ${ }^{\star *}$} \\
\hline $1 \mathrm{~cm} / \mathrm{in}^{*}$ & $8,388.7$ & 903.9 & $1,783.4$ & $1,286.7$ & 38252 & 3477.5 \\
\hline $1 \mathrm{ft}$ & 693.2 & 74.7 & 146.2 & 105.5 & 3161.0 & 287.4 \\
\hline $1 \mathrm{~m}$ & 131.7 & 14.2 & 27.9 & 20.2 & 600.5 & 54.6 \\
\hline $10 \mathrm{ft}$ & 15.6 & 1.7 & 3.4 & 2.4 & 71.1 & 6.5 \\
\hline
\end{tabular}

* Dose rates calculated using Microshield over estimate dose rates at very close distances $\leq 1$ inch to the source, and should be evaluated closely prior to use.

** Unshielded sludge sample hose dose rates were determined by ratio of the ALARA report and Microshield calculation done for this assessment, the most conservative value of 4.56 was used to determine settler sludge unshielded sludge sample hose (USSL) dose rates.

***It is unlikely any dose rates will meet or exceed the calculated values, this calculation employs very conservative source terms and geometries

${ }_{* \star * *}$ Results are from KBC-37418 and are for comparison to results found in Microshield KBC40179_1 and 2 to verify validity of model.

Using the information provided in Table 3 above, the values have been incorporated into the following tables so that the total collective doses for the workers can be compared for above water sampling of container sampling and settler sampling. The spreadsheet was developed initially to calculate the collective dose for the container sampling activities (see letter 09-100K-GLH-003). The tables below utilize the same methodology, quantity of personnel, and times for the settler sampling, only substituting dose rates calculated for settler sludge to determine the total collective dose. 
Table 4

Collective Dose for Preparation of the Work Area and Setup of Sampling Equipment

\begin{tabular}{|c|c|c|}
\hline & NCO's & 5 \\
\hline & RCT's & 2 \\
\hline & FWS & 1 \\
\hline & Engineer & 1 \\
\hline & TOTAL & 9 \\
\hline \multicolumn{3}{|l|}{ This does not take into account the } \\
\hline installation of the Extraction/lsolation & Hours per day for this activity $=$ & 1.0 \\
\hline tubes. Which is performed under a & Shifts worked per day $=$ & 1 \\
\hline \multirow[t]{5}{*}{ separate work package. } & Total Days Worked = & 5 \\
\hline & Total Person-Hours = & 45 \\
\hline & Highest Projected Dose Rate $(2 \mathrm{mr} / \mathrm{hr})$ assigned $(2$ minutes $)=$ & 3 mrem \\
\hline & Average Projected Dose Rate $(1 \mathrm{mr} / \mathrm{hr})$ assigned $(8$ minutes $)=$ & 6 mrem \\
\hline & $\begin{array}{c}\text { Lowest Projected Dose Rate }(0.5 \mathrm{mr} / \mathrm{hr}) \text { assigned }(50 \text { minutes })= \\
\text { Person-Dose in mrem }=\end{array}$ & $\begin{array}{l}19 \text { mrem } \\
28\end{array}$ \\
\hline
\end{tabular}

Table 5

Collective Dose for Sample Collection

Supports the collection of 2 samples/day, changing out

of bottle cap assembly unit, transferring of sample bottles

to SSC, and transfer of SSC to Storage/Staging area.

$\begin{array}{ll}\text { NCO's } & 5 \\ \text { RCT'S } & 2\end{array}$

FWS 1

Engineer 1

TOTAL 9

Hours per day for this activity $=\quad 2.0$

Shifts worked per day $=\quad 1$

Total Days Worked $=\quad 5$

Total Person-Hours $=\quad 90$

Highest Projected Dose Rate $(415 \mathrm{mr} / \mathrm{hr})$ assigned $(2$ minutes $)=1245 \mathrm{mrem}$ Average Projected Dose Rate $(50.7 \mathrm{mr} / \mathrm{hr})$ assigned $(8$ minutes $)=608 \mathrm{mrem}$ Lowest Projected Dose Rate $(2 \mathrm{mr} / \mathrm{hr})$ assigned $(50$ minutes $)=150 \mathrm{mrem}$

Person-Dose in mrem $=$

\section{Table 6}

\section{Collective Dose for Sample Measurement and Shipment}

If core is completed, remove $T$-handle from extraction tube.

Confirm sludge depth, lift sample bottle from SSC

after 12 hrs, measure sludge height, and perform

required surveys.

\begin{tabular}{cc|} 
NCO's & 5 \\
RCT's & 2 \\
FWS & 1 \\
Engineer & 1 \\
TOTAL & 9 \\
Hours per day for this activity $=$ & 1.0 \\
Shifts worked per day $=$ & 1 \\
Total Days Worked & \\
( Total Person-Hours $=$ & 45 \\
Rate $(415 \mathrm{mr} / \mathrm{hr})$ assigned $(2 \mathrm{minutes})=$ & $622 \mathrm{mrem}$ \\
Rate $(50.7 \mathrm{mr} / \mathrm{hr})$ assigned $(2 \mathrm{minutes})=$ & $76 \mathrm{mrem}$ \\
Person-Dose in mrem = & $84 \mathrm{mrem}$ \\
\hline Rate $(2 \mathrm{mr} / \mathrm{hr})$ assigned $(56$ minutes $)=$ & 782
\end{tabular}

Highest Projected Dose Rate $(415 \mathrm{mr} / \mathrm{hr})$ assigned (2 minutes) $=$ Average Projected Dose Rate $(50.7 \mathrm{mr} / \mathrm{hr})$ assigned $(2$ minutes $)=$ Lowest Projected Dose Rate $(2 \mathrm{mr} / \mathrm{hr})$ assigned (56 minutes) $=$

Person-Dose in mrem $=$ 
Table 7

Collective Dose for Contingency Work

Assume an extra 30 days for any additional

installation of extraction and isolation tubes, complications,

or work delays, which do not perform sludge sampling.

NCO's
RCT's
FWS
Engineer
TOTAL

Hours per day for this activity $=$ Shifts worked per day $=$

Total Days Worked $=$

Total Person-Hours $=$

Highest Projected Dose Rate $(2 \mathrm{mr} / \mathrm{hr})$ assigned $(2$ minutes $)=$ Average Projected Dose Rate $(1 \mathrm{mr} / \mathrm{hr})$ assigned $(8$ minutes $)=$ Lowest Projected Dose Rate $(0.5 \mathrm{mr} / \mathrm{hr})$ assigned $(50$ minutes $)=$

Person-Dose in mrem =
1

1

9

4.0

1

30

1080

72 mrem

144 mrem

450 mrem

666

Note: contingency dose accounts for work stoppages, delays, additional samples (not additional sample volume).

\section{Table 8}

\section{Collective Dose Results}

- $\quad$ Assumes 10 samples pulled from Container SCS-CON-230.

- $\quad$ Assume a maximum of 5 days to pull 10 sludge samples.

- Average basin entry time for workers is $\sim 4 \mathrm{hr} / \mathrm{shift}$

- Assume an extra 30 days for any additional installation of extraction and isolation tubes, complications, additional samples or work delays.

- Assume only day shift will support the KW Basin Sludge Sampling campaign.

- $\quad$ Spreadsheet is based letter 09-100KGLH-003

\begin{tabular}{|l|c|}
\multicolumn{1}{|c|}{ Activity } & Collective Dose \\
\hline $\begin{array}{l}\text { Preparation of the Work Area and Setup of } \\
\text { Sampling Equipment - Table 4 }\end{array}$ & 28 person-mrem \\
\hline Sample Collection - Table 5 & 2003 person-mrem \\
\hline $\begin{array}{l}\text { Sample Measurement and Shipment - } \\
\text { Table 6 }\end{array}$ & 782 person-mrem \\
\hline Contingency Work - Table 7 & 666 person-mrem \\
\hline Total Collective Dose & 3479 person-mrem \\
\hline Average Dose per Sample & 348 person-mrem \\
& \\
\hline Total person-hours & 1260 hours \\
& \\
\hline
\end{tabular}


Table 9

COST COMPARISON OF OLD PLANNED VS NEW PLAN (UTILIZING EXISTING DESIGN)

\begin{tabular}{|c|c|c|c|c|}
\hline ACTIVITY DESCRIPTION & & $\begin{array}{c}\text { ROM } \\
\text { TOTAL COST } \\
\end{array}$ & $\begin{array}{l}\text { REDUCED } \\
\text { RETRIEVAL } \\
\end{array}$ & $\begin{array}{c}\text { STILL } \\
\text { NEEDED } \\
\text { FOR } \\
\text { SAMPLING } \\
\end{array}$ \\
\hline & & RETRIEVAL/SAMPLING & SAMPLING & NOW \\
\hline SETTLER RETRIEVAL/SAMPLING BASIS OF ESTIMATE & RET/SAM & Date $10 / 07$ & Date $10 / 07$ & Date 2/09 \\
\hline $\begin{array}{l}\text { STS0005/STS0010, Engineering Work Plans } \\
\text { STT0465-STTT0525 Conceptual Design Retrieval Equipment for } \\
\text { Sample } \\
\text { STS0025,Conceptual Design for Settler Sampling } \\
\text { STT0530, 535, } 540 \text { Review and Approve Test and Conceptual } \\
\text { Design } \\
\text { STS0030, Finalize Settler Retrieval/Sampling Design } \\
\text { STS0230, Design Review and Technical Support } \\
\text { STS0035 KW Basin Prep/JCS wk pkg/ Operating Procedures } \\
\text { STS0170/STS0180/STS0027 Hazard Analysis } \\
\text { STS0185 USQ } \\
\text { STS0040 Fab/Procure Retrieval/Sampling Equip } \\
\text { STS0045 Install/Prep Equipment in basin for Settler } \\
\text { STS0195 Readiness Review (MSA) }\end{array}$ & $\begin{array}{l}\text { RET/SAM } \\
\text { RET } \\
\text { SAMP } \\
\text { RET/SAM } \\
\text { RET/SAM } \\
\text { RET/SAM } \\
\text { elsewhere } \\
\text { elsewhere } \\
\text { RET/SAM } \\
\text { RET/SAM } \\
\text { RET/SAM }\end{array}$ & $\begin{array}{c}\$ 0 \\
\$ 518,200 \\
\$ 400,000 \\
\\
\$ 761,200 \\
\$ 70,500 \\
\$ 78,300 \\
\$ 0 \\
\$ 0 \\
\$ 518,000 \\
\$ 702,000 \\
\$ 324,000\end{array}$ & $\begin{array}{c}\$ 0 \\
\$ 0 \\
\$ 400,000 \\
\$ 0 \\
\$ 380,600 \\
\$ 35,250 \\
\$ 39,150 \\
\$ 0 \\
\$ 0 \\
\$ 259,000 \\
\$ 351,000 \\
\$ 162,000\end{array}$ & $\begin{array}{c}0 \\
0 \\
0 \\
0 \\
\$ 67,320 \\
10,200 \\
\$ 30,600 \\
0 \\
0 \\
192,000 \\
220,320 \\
0\end{array}$ \\
\hline $\begin{array}{l}\text { STS0050 Retrieve Settler sludge } \\
\text { STT0355, Sample Settler Container and transport }\end{array}$ & $\begin{array}{l}\text { RET } \\
\text { SAMP }\end{array}$ & $\begin{array}{l}\$ 270,000 \\
\$ 350,000\end{array}$ & $\begin{array}{c}\$ 0 \\
\$ 350,000 \\
\end{array}$ & $\begin{array}{c}0 \\
\$ 350,000\end{array}$ \\
\hline SETTLER SLUDGE SAMPLING SUBTOTAL & & $\$ 3,992,200$ & $\$ 1,977,000$ & $\$ 870,440$ \\
\hline TOTAL NET SAVINGS & & & & $\$ 1,106,560$ \\
\hline
\end{tabular}

\begin{tabular}{|c|c|c|c|c|c|}
\hline BASIS OF NEW ESTIMATE: & FTE & DAYS & $\begin{array}{c}\text { COST PER } \\
\text { HOUR }\end{array}$ & $\begin{array}{c}\text { HOUR } \\
\text { PER DAY }\end{array}$ & $\begin{array}{l}\text { TOTAL } \\
\text { COST }\end{array}$ \\
\hline \multirow{12}{*}{$\begin{array}{l}\text { FMP NEEDED TO SHOW CONFIGURATION TOP OF SCS-CON- } \\
230 \\
\text { DESIGN REVIEW ( ASSSUME NO REVISION TO } \\
\text { THERMAL/GAS) } \\
\text { WORK PACKAGE DEVELOPMENT/AJHAIEWPNWALKDOWNS } \\
\text { FAB PROCURE EQUIPMENT } \\
\text { SHIPPING PIGS } 6 \text { X } 12,000 \\
\text { ISOLATIONS TUBES/EXTRACTION TUBES } 4 \text { EACH } \\
\text { GUIDE TUBES (4 EACH) } \\
\text { SAMPLE BAG/BOTTLES, CLEANING STATIONS (24) } \\
\text { WORK PLATFORM AND FRAMES AND COVERS } \\
\text { TUBING ASSEMBLIES/NALVES } \\
\text { SPARES (20) /UNKNOWS (20) } \\
\text { INSTALLATION ASSUME } 18 \text { DAYS + } 3 \text { CM/SAF/FWS } \\
\text { SYSTEM OPERATION }\end{array}$} & \multirow{3}{*}{$\begin{array}{c}3 \\
15 \\
1.5\end{array}$} & 33 & $\$ 85.00$ & 8 & $\$ 67,320$ \\
\hline & & \multirow{3}{*}{$\begin{array}{c}1 \\
30\end{array}$} & \multirow{2}{*}{$\begin{array}{l}\$ 85.00 \\
\$ 85.00\end{array}$} & \multirow{3}{*}{$\begin{array}{l}8 \\
8\end{array}$} & $\$ 10,200$ \\
\hline & & & & & $\$ 30,600$ \\
\hline & & & & & $\$ 0$ \\
\hline & & & & & $\$ 68,000$ \\
\hline & & & & & $\$ 12,000$ \\
\hline & & & & & $\$ 12,000$ \\
\hline & & & & & $\$ 10,000$ \\
\hline & & & & & $\$ 25,000$ \\
\hline & & & & & $\begin{array}{l}\$ 25,000 \\
\$ 40,000\end{array}$ \\
\hline & 18 & 18 & $\$ 85.00$ & 8 & $\$ 220,320$ \\
\hline & & & & & $\$ 350,000$ \\
\hline TOTALS & & & & & $\$ 870,440$ \\
\hline
\end{tabular}

This assumes the same system that was used for container sampling will be used for settler sampling. No 325 Building Cost, Sample receipt cost or Lab analysis cost are Included in either estimate. 


\section{Cost/Benefit Analysis:}

The total collective dose anticipated for settler sampling, Table 8 above, is 3.479 person-rem, with the average per sample dose being 0.348 person-rem. This dose compares to the total anticipated collective dose for the container sludge sampling using similar sampling methods, of 5.453 person-rem, with the average per sample dose being 0.101 person-rem.

For the purpose of this assessment it is assumed that the retrieval of settler sludge via the underwater method will result in no personnel dose (most conservative estimate possible). Therefore, the cost-benefit analysis will use a total calculated person-dose for above water settler sludge sampling of 3.479 personrem. The ALARA dose decision value for optimization is procedurally established at $\$ 25,000$ /person-rem (per PRO-24889). Therefore, expenditure of funds of up to $\$ 86,975$ could be justified to avoid an estimated collective dose of 3.479 person-rem.

As a validation measure the same assessment was preformed above not assuming any dose reduction factor (ie 4569 vs. 415 and 557.7 vs. 50.7 , a very conservative assumption), this resulted in a total dose of approximately 28.9 person-rem and a dollar value of $\$ 724,850$, again well below the anticipated cost savings.

The change to this sampling method does not mean that the project will not incorporate ALARA concepts as the sampling campaign advances or to mitigate doses as the design evolves and sampling information is accumulated, with that in mind, the following item has been discussed by the project for possible modification prior to initiating settler sampling:

Extension of the sampling T-bar (see figure 6) presently provides 2 feet on each side of the J-hook, it is assumed that this provides a total of 3 foot to the worker, if the T-bar was extended to 4 foot on each side such that it provided a total of 5 feet of distance it would significantly reduce the doses to workers from even the data book sludge values and would be appropriate as an ALARA feature for movement of higher dose rate samples (see KBC-40179_6 for data book dose rates, these are plotted on table 10 below)

\section{Conclusion:}

While dose rates for the unshielded settler sludge samples are anticipated to be higher than container sludge sampling, the collective dose is measurably lower. Controls that have been or that will be put in place for container sludge sampling will help further reduce collective doses, and by using the same sampling technique for settler sludge, operational lessons learned can be applied. Additionally, using the sampling process that operators have experience with could contribute to fewer unanticipated radiological events, which in themselves can be a significant contributor to workers collective dose.

The current STP project cost projections to design and construct underwater settler sludge sampling equipment is estimated to be $\$ 1,977,000$ (per table 9 above). This compares to a cost estimate using the container sludge sampling system of $\$ 870,440$ (a reduction of $\$ 1,106,560$ ) this additional cost cannot be justified for the sole purpose of personnel dose reduction. It is understood that there may be other operational considerations (they are not evaluated in this assessment) that may warrant underwater sampling of the settler sludge, but the ALARA consideration for dose should not be a deciding factor in the path forward for settler sludge sampling. 
Figure 6

T-bar Assemble

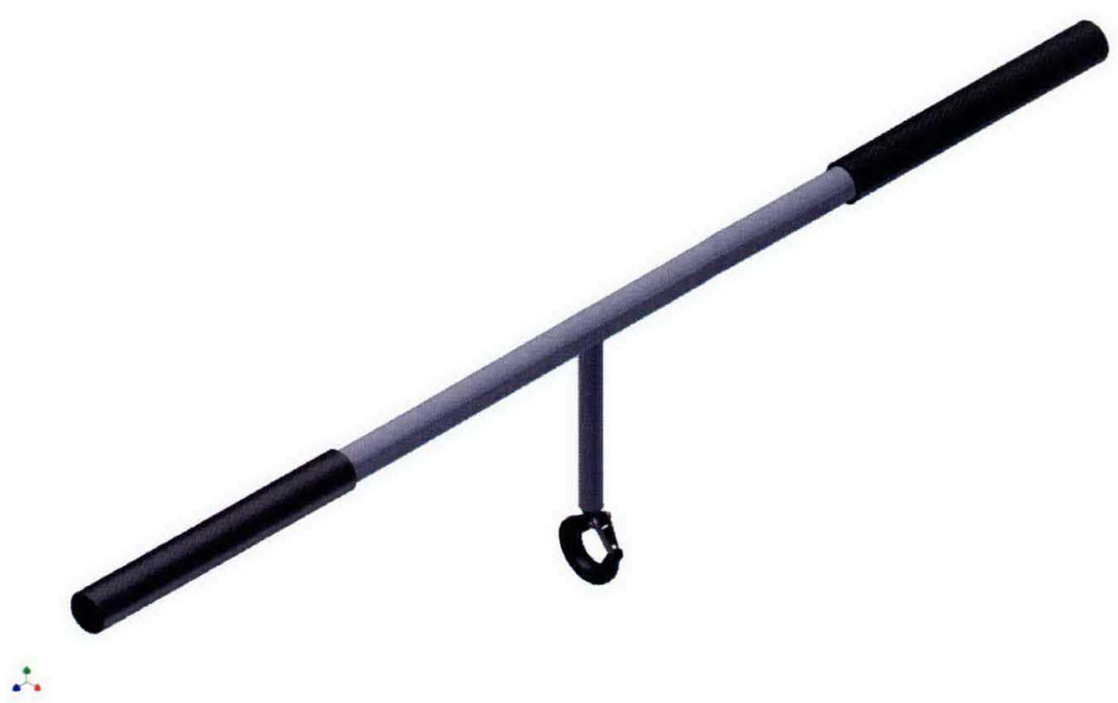

Table 10

Data Book Values Dose Rates of $400 \mathrm{ml}$ of Settler Sludge versus Distance (Worst Case Sludge)

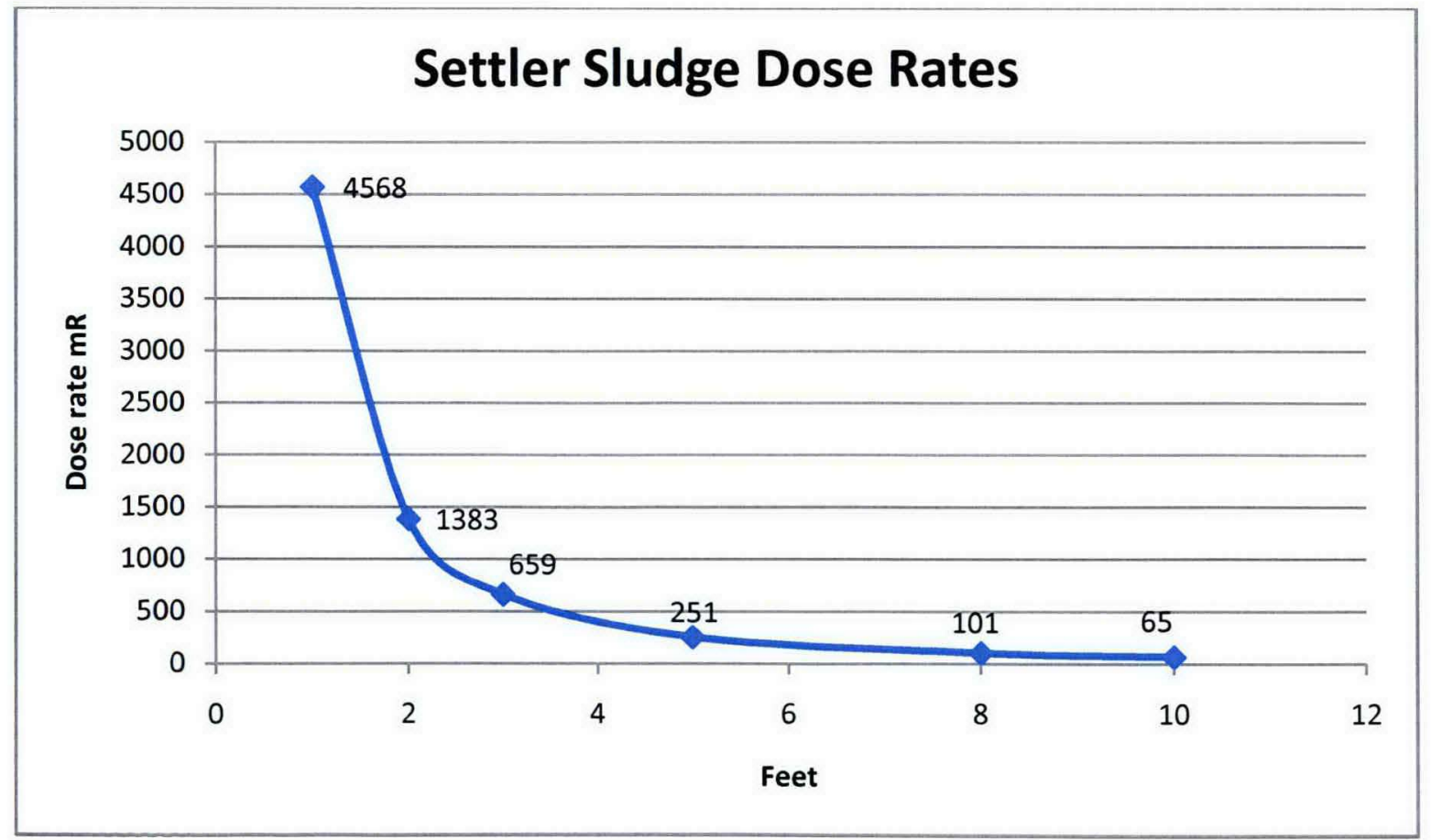




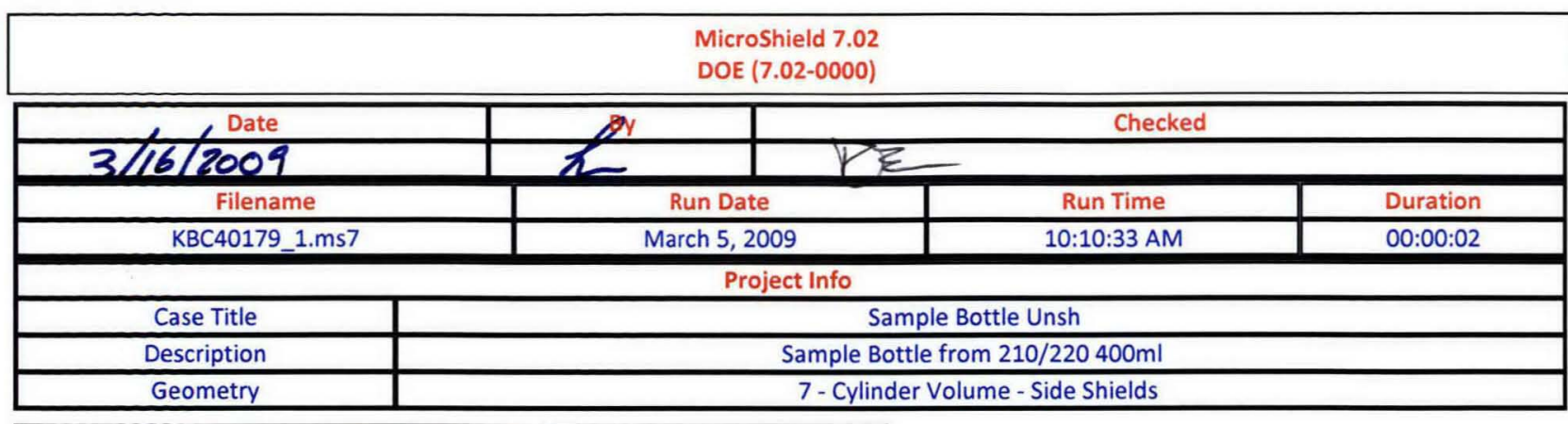

\begin{tabular}{|c|c|c|c|c|}
\hline \multicolumn{5}{|c|}{ Source Dimensions } \\
\hline & Height & \multicolumn{3}{|c|}{$2.195 \mathrm{~cm}(0.9 \mathrm{in})$} \\
\hline & Radius & \multicolumn{3}{|c|}{$7.62 \mathrm{~cm}$ (3.0 in) } \\
\hline \multicolumn{5}{|c|}{ Dose Points } \\
\hline A & \multicolumn{2}{|c|}{$\mathrm{x}$} & $\mathrm{Y}$ & $\mathrm{z}$ \\
\hline$\# 1$ & \multicolumn{2}{|c|}{$10.638 \mathrm{~cm}(4.2 \mathrm{in})$} & $1.1 \mathrm{~cm}(0.4 \mathrm{in})$ & $0.0 \mathrm{~cm}(0.0 \mathrm{in})$ \\
\hline$\# 2$ & \multicolumn{2}{|c|}{$38.578 \mathrm{~cm}(1 \mathrm{ft} 3.2 \mathrm{in})$} & $1.1 \mathrm{~cm}(0.4 \mathrm{in})$ & $0.0 \mathrm{~cm}(0.0 \mathrm{in})$ \\
\hline$\# 3$ & \multicolumn{2}{|c|}{$108.1 \mathrm{~cm}(3 \mathrm{ft} 6.6 \mathrm{in})$} & $1.1 \mathrm{~cm}(0.4 \mathrm{in})$ & $0.0 \mathrm{~cm}(0.0 \mathrm{in})$ \\
\hline \#4 & \multicolumn{2}{|c|}{$312.898 \mathrm{~cm}(10 \mathrm{ft} 3.2 \mathrm{in})$} & $1.1 \mathrm{~cm}(0.4 \mathrm{in})$ & $0.0 \mathrm{~cm}(0.0 \mathrm{in})$ \\
\hline \multicolumn{5}{|c|}{ Shields } \\
\hline & Shield N & Dimension & Material & Density \\
\hline & Source & $400.4 \mathrm{~cm}^{3}$ & Water & 1.59 \\
\hline & Transition & & Air & 0.00122 \\
\hline & Air Gap & & Air & 0.00122 \\
\hline & Wall Clad & $.478 \mathrm{~cm}$ & Water & 1 \\
\hline & Top Clad & $.478 \mathrm{~cm}$ & Water & 1 \\
\hline
\end{tabular}

\begin{tabular}{|c|c|c|c|c|}
\hline \multicolumn{5}{|c|}{$\begin{array}{c}\text { Source Input: Grouping Method - Linear Energy } \\
\text { Number of Groups: } 25 \\
\text { Lower Energy Cutoff: } 0.015 \\
\text { Photons < } 0.015: \text { Excluded } \\
\text { Library: Grove } \\
\end{array}$} \\
\hline Nuclide & $\mathrm{Ci}$ & $\mathrm{Bq}$ & $\mu \mathrm{Ci} / \mathrm{cm}^{3}$ & $\mathrm{~Bq} / \mathrm{cm}^{3}$ \\
\hline$A m-241$ & $2.7027 e-002$ & $1.0000 \mathrm{e}+009$ & $6.7500 \mathrm{e}+001$ & $2.4975 e+006$ \\
\hline Ba-137m & $4.8484 \mathrm{e}-001$ & $1.7939 \mathrm{e}+010$ & $1.2109 \mathrm{e}+003$ & $4.4803 e+007$ \\
\hline Co-60 & $4.4044 \mathrm{e}-004$ & $1.6296 \mathrm{e}+007$ & $1.1000 \mathrm{e}+000$ & $4.0700 \mathrm{e}+004$ \\
\hline Cs-134 & $1.0050 \mathrm{e}-003$ & $3.7185 e+007$ & $2.5100 \mathrm{e}+000$ & $9.2870 \mathrm{e}+004$ \\
\hline Cs-137 & $5.1251 \mathrm{e}-001$ & $1.8963 e+010$ & $1.2800 \mathrm{e}+003$ & $4.7360 \mathrm{e}+007$ \\
\hline Eu-154 & $3.7518 \mathrm{e}-003$ & $1.3881 \mathrm{e}+008$ & $9.3700 \mathrm{e}+000$ & $3.4669 e+005$ \\
\hline Eu-155 & $1.1171 \mathrm{e}-003$ & $4.1333 e+007$ & $2.7900 \mathrm{e}+000$ & $1.0323 e+005$ \\
\hline $\mathrm{Np}-237$ & $2.8869 \mathrm{e}-006$ & $1.0681 \mathrm{e}+005$ & $7.2100 \mathrm{e}-003$ & $2.6677 \mathrm{e}+002$ \\
\hline Pu-238 & $4.6446 \mathrm{e}-003$ & $1.7185 e+008$ & $1.1600 \mathrm{e}+001$ & $4.2920 \mathrm{e}+005$ \\
\hline Pu-239 & $1.6496 \mathrm{e}-002$ & $6.1037 e+008$ & $4.1200 \mathrm{e}+001$ & $1.5244 \mathrm{e}+006$ \\
\hline Pu-240 & $1.0010 \mathrm{e}-002$ & $3.7037 e+008$ & $2.5000 \mathrm{e}+001$ & $9.2500 \mathrm{e}+005$ \\
\hline Pu-241 & $4.1642 \mathrm{e}-001$ & $1.5407 \mathrm{e}+010$ & $1.0400 \mathrm{e}+003$ & $3.8480 \mathrm{e}+007$ \\
\hline $\mathrm{Pu}-242$ & $4.0440 \mathrm{e}-006$ & $1.4963 e+005$ & $1.0100 \mathrm{e}-002$ & $3.7370 \mathrm{e}+002$ \\
\hline$S m-151$ & $1.0130 \mathrm{e}-003$ & $3.7481 \mathrm{e}+007$ & $2.5300 \mathrm{e}+000$ & $9.3610 \mathrm{e}+004$ \\
\hline Sr-90 & $5.0450 \mathrm{e}-001$ & $1.8667 e+010$ & $1.2600 \mathrm{e}+003$ & $4.6620 \mathrm{e}+007$ \\
\hline Tc-99 & $1.3173 e-004$ & $4.8741 \mathrm{e}+006$ & $3.2900 \mathrm{e}-001$ & $1.2173 e+004$ \\
\hline Y-90 & $5.0450 \mathrm{e}-001$ & $1.8667 \mathrm{e}+010$ & $1.2600 \mathrm{e}+003$ & $4.6620 \mathrm{e}+007$ \\
\hline \multicolumn{5}{|c|}{$\begin{array}{l}\text { Buildup: The material reference is Source } \\
\text { Integration Parameters }\end{array}$} \\
\hline \multicolumn{4}{|c|}{ Radial } & 20 \\
\hline \multicolumn{4}{|c|}{ Circumferential } & 20 \\
\hline \multicolumn{4}{|c|}{ Y Direction (axial) } & 20 \\
\hline \multicolumn{5}{|c|}{ Results - Dose Point \# 1 - $(10.63752,1.1,0) \mathrm{cm}$} \\
\hline
\end{tabular}




\begin{tabular}{|c|c|c|c|c|c|}
\hline Energy (MeV) & Activity (Photons/sec) & $\begin{array}{l}\text { Fluence Rate } \\
\mathrm{MeV} / \mathrm{cm}^{2} / \mathrm{sec} \\
\text { No Buildup }\end{array}$ & $\begin{array}{l}\text { Fluence Rate } \\
\mathrm{MeV} / \mathrm{cm}^{2} / \mathrm{sec} \\
\text { With Buildup }\end{array}$ & $\begin{array}{c}\text { Exposure Rate } \\
\text { mR/hr } \\
\text { No Buildup }\end{array}$ & $\begin{array}{c}\text { Exposure Rate } \\
\mathrm{mR} / \mathrm{hr} \\
\text { With Buildup }\end{array}$ \\
\hline 0.043 & $1.826 \mathrm{e}+09$ & $2.181 e+04$ & $7.426 e+04$ & $8.047 e+01$ & $2.740 \mathrm{e}+02$ \\
\hline 0.5923 & $5.491 \mathrm{e}+07$ & $1.726 \mathrm{e}+04$ & $2.832 \mathrm{e}+04$ & $3.372 \mathrm{e}+01$ & $5.531 \mathrm{e}+01$ \\
\hline 0.6618 & $1.617 \mathrm{e}+10$ & $5.821 e+06$ & $9.223 e+06$ & $1.128 \mathrm{e}+04$ & $1.788 \mathrm{e}+04$ \\
\hline 0.7924 & $4.274 \mathrm{e}+07$ & $1.915 \mathrm{e}+04$ & $2.880 e+04$ & $3.647 \mathrm{e}+01$ & $5.484 \mathrm{e}+01$ \\
\hline 0.8759 & $1.775 e+07$ & $8.980 \mathrm{e}+03$ & $1.317 e+04$ & $1.689 \mathrm{e}+01$ & $2.477 e+01$ \\
\hline 1.0017 & $3.912 \mathrm{e}+07$ & $2.329 e+04$ & $3.314 e+04$ & $4.292 \mathrm{e}+01$ & $6.106 \mathrm{e}+01$ \\
\hline 1.0416 & $5.689 e+05$ & $3.551 \mathrm{e}+02$ & $5.008 \mathrm{e}+02$ & $6.498 \mathrm{e}-01$ & $9.165 \mathrm{e}-01$ \\
\hline 1.1185 & $1.429 \mathrm{e}+05$ & $9.718 \mathrm{e}+01$ & $1.349 \mathrm{e}+02$ & $1.754 \mathrm{e}-01$ & $2.435 \mathrm{e}-01$ \\
\hline 1.1509 & $1.339 e+06$ & $9.429 e+02$ & $1.300 \mathrm{e}+03$ & $1.692 \mathrm{e}+00$ & $2.333 e+00$ \\
\hline 1.1732 & $1.630 e+07$ & $1.174 e+04$ & $1.612 e+04$ & $2.098 \mathrm{e}+01$ & $2.881 \mathrm{e}+01$ \\
\hline 1.2456 & $1.429 e+06$ & $1.106 \mathrm{e}+03$ & $1.499 \mathrm{e}+03$ & $1.950 \mathrm{e}+00$ & $2.644 \mathrm{e}+00$ \\
\hline 1.2745 & $4.927 e+07$ & $3.921 \mathrm{e}+04$ & $5.288 \mathrm{e}+04$ & $6.876 e+01$ & $9.274 \mathrm{e}+01$ \\
\hline 1.3325 & $1.630 \mathrm{e}+07$ & $1.368 \mathrm{e}+04$ & $1.828 \mathrm{e}+04$ & $2.373 e+01$ & $3.171 \mathrm{e}+01$ \\
\hline 1.3652 & $1.130 \mathrm{e}+06$ & $9.768 \mathrm{e}+02$ & $1.299 \mathrm{e}+03$ & $1.684 \mathrm{e}+00$ & $2.240 \mathrm{e}+00$ \\
\hline 1.4944 & $9.017 e+05$ & $8.681 \mathrm{e}+02$ & $1.134 \mathrm{e}+03$ & $1.462 \mathrm{e}+00$ & $1.910 \mathrm{e}+00$ \\
\hline 1.5952 & $3.991 \mathrm{e}+06$ & $4.153 e+03$ & $5.361 \mathrm{e}+03$ & $6.869 \mathrm{e}+00$ & $8.868 \mathrm{e}+00$ \\
\hline Totals & $1.825 e+10$ & $5.984 e+06$ & $9.499 \mathrm{e}+06$ & $1.162 \mathrm{e}+04$ & $1.852 \mathrm{e}+04$ \\
\hline \multicolumn{6}{|c|}{ Results - Dose Point \# 2 - $(38.57752,1.1,0) \mathrm{cm}$} \\
\hline Energy (MeV) & Activity (Photons/sec) & $\begin{array}{c}\text { Fluence Rate } \\
\mathrm{MeV} / \mathrm{cm}^{2} / \mathrm{sec} \\
\text { No Buildup }\end{array}$ & $\begin{array}{l}\text { Fluence Rate } \\
\mathrm{MeV} / \mathrm{cm}^{2} / \mathrm{sec} \\
\text { With Buildup }\end{array}$ & $\begin{array}{c}\text { Exposure Rate } \\
\mathrm{mR} / \mathrm{hr} \\
\text { No Buildup } \\
\end{array}$ & $\begin{array}{c}\text { Exposure Rate } \\
\mathrm{mR} / \mathrm{hr} \\
\text { With Buildup }\end{array}$ \\
\hline 0.043 & $1.826 \mathrm{e}+09$ & $8.677 \mathrm{e}+02$ & $3.515 e+03$ & $3.202 \mathrm{e}+00$ & $1.297 \mathrm{e}+01$ \\
\hline 0.5923 & $5.491 \mathrm{e}+07$ & $8.139 e+02$ & $1.518 \mathrm{e}+03$ & $1.590 \mathrm{e}+00$ & $2.964 \mathrm{e}+00$ \\
\hline 0.6618 & $1.617 \mathrm{e}+10$ & $2.763 e+05$ & $4.937 e+05$ & $5.357 \mathrm{e}+02$ & $9.571 \mathrm{e}+02$ \\
\hline 0.7924 & $4.274 \mathrm{e}+07$ & $9.193 e+02$ & $1.540 \mathrm{e}+03$ & $1.751 \mathrm{e}+00$ & $2.932 \mathrm{e}+00$ \\
\hline 0.8759 & $1.775 \mathrm{e}+07$ & $4.337 e+02$ & $7.040 \mathrm{e}+02$ & $8.156 \mathrm{e}-01$ & $1.324 \mathrm{e}+00$ \\
\hline 1.0017 & $3.912 \mathrm{e}+07$ & $1.134 \mathrm{e}+03$ & $1.771 \mathrm{e}+03$ & $2.089 \mathrm{e}+00$ & $3.264 \mathrm{e}+00$ \\
\hline 1.0416 & $5.689 e+05$ & $1.733 e+01$ & $2.677 e+01$ & $3.171 \mathrm{e}-02$ & $4.900 \mathrm{e}-02$ \\
\hline 1.1185 & $1.429 e+05$ & $4.762 \mathrm{e}+00$ & $7.212 e+00$ & $8.596 \mathrm{e}-03$ & $1.302 \mathrm{e}-02$ \\
\hline 1.1509 & $1.339 \mathrm{e}+06$ & $4.628 \mathrm{e}+01$ & $6.953 e+01$ & $8.304 \mathrm{e}-02$ & $1.248 \mathrm{e}-01$ \\
\hline 1.1732 & $1.630 \mathrm{e}+07$ & $5.768 \mathrm{e}+02$ & $8.621 \mathrm{e}+02$ & $1.031 \mathrm{e}+00$ & $1.541 \mathrm{e}+00$ \\
\hline 1.2456 & $1.429 e+06$ & $5.454 \mathrm{e}+01$ & $8.019 e+01$ & $9.617 e-02$ & $1.414 \mathrm{e}-01$ \\
\hline 1.2745 & $4.927 e+07$ & $1.936 \mathrm{e}+03$ & $2.828 \mathrm{e}+03$ & $3.395 e+00$ & $4.960 \mathrm{e}+00$ \\
\hline 1.3325 & $1.630 \mathrm{e}+07$ & $6.771 \mathrm{e}+02$ & $9.777 e+02$ & $1.175 e+00$ & $1.696 \mathrm{e}+00$ \\
\hline 1.3652 & $1.130 \mathrm{e}+06$ & $4.842 e+01$ & $6.948 \mathrm{e}+01$ & $8.349 \mathrm{e}-02$ & $1.198 \mathrm{e}-01$ \\
\hline 1.4944 & $9.017 e+05$ & $4.324 \mathrm{e}+01$ & $6.068 \mathrm{e}+01$ & $7.282 \mathrm{e}-02$ & $1.022 \mathrm{e}-01$ \\
\hline 1.5952 & $3.991 \mathrm{e}+06$ & $2.076 \mathrm{e}+02$ & $2.870 \mathrm{e}+02$ & $3.434 \mathrm{e}-01$ & $4.748 \mathrm{e}-01$ \\
\hline Totals & $1.825 e+10$ & $2.841 e+05$ & $5.080 e+05$ & $5.515 e+02$ & $9.898 \mathrm{e}+02$ \\
\hline \multicolumn{6}{|c|}{ Results - Dose Point \# 3 - $(108.1,1.1,0) \mathrm{cm}$} \\
\hline Energy (MeV) & Activity (Photons/sec) & $\begin{array}{c}\text { Fluence Rate } \\
\mathrm{MeV} / \mathrm{cm}^{2} / \mathrm{sec} \\
\text { No Buildup }\end{array}$ & $\begin{array}{l}\text { Fluence Rate } \\
\mathrm{MeV} / \mathrm{cm}^{2} / \mathrm{sec} \\
\text { With Buildup }\end{array}$ & $\begin{array}{c}\text { Exposure Rate } \\
\text { mR/hr } \\
\text { No Buildup }\end{array}$ & $\begin{array}{c}\text { Exposure Rate } \\
\mathrm{mR} / \mathrm{hr} \\
\text { With Buildup }\end{array}$ \\
\hline 0.043 & $1.826 \mathrm{e}+09$ & $1.001 \mathrm{e}+02$ & $4.231 \mathrm{e}+02$ & $3.693 \mathrm{e}-01$ & $1.561 \mathrm{e}+00$ \\
\hline 0.5923 & $5.491 \mathrm{e}+07$ & $9.801 \mathrm{e}+01$ & $1.881 \mathrm{e}+02$ & $1.914 \mathrm{e}-01$ & $3.675 e-01$ \\
\hline 0.6618 & $1.617 \mathrm{e}+10$ & $3.333 e+04$ & $6.119 e+04$ & $6.461 \mathrm{e}+01$ & $1.186 \mathrm{e}+02$ \\
\hline 0.7924 & $4.274 \mathrm{e}+07$ & $1.112 \mathrm{e}+02$ & $1.908 \mathrm{e}+02$ & $2.117 \mathrm{e}-01$ & $3.633 e-01$ \\
\hline 0.8759 & $1.775 e+07$ & $5.252 \mathrm{e}+01$ & $8.724 \mathrm{e}+01$ & $9.877 \mathrm{e}-02$ & $1.641 \mathrm{e}-01$ \\
\hline 1.0017 & $3.912 \mathrm{e}+07$ & $1.376 \mathrm{e}+02$ & $2.195 e+02$ & $2.535 \mathrm{e}-01$ & $4.045 \mathrm{e}-01$ \\
\hline 1.0416 & $5.689 \mathrm{e}+05$ & $2.103 e+00$ & $3.318 \mathrm{e}+00$ & $3.849 \mathrm{e}-03$ & $6.072 \mathrm{e}-03$ \\
\hline 1.1185 & $1.429 \mathrm{e}+05$ & $5.787 \mathrm{e}-01$ & $8.939 \mathrm{e}-01$ & $1.045 \mathrm{e}-03$ & $1.613 e-03$ \\
\hline 1.1509 & $1.339 \mathrm{e}+06$ & $5.626 e+00$ & $8.618 \mathrm{e}+00$ & $1.010 \mathrm{e}-02$ & $1.546 \mathrm{e}-02$ \\
\hline 1.1732 & $1.630 \mathrm{e}+07$ & $7.014 \mathrm{e}+01$ & $1.068 \mathrm{e}+02$ & $1.253 e-01$ & $1.909 \mathrm{e}-01$ \\
\hline 1.2456 & $1.429 \mathrm{e}+06$ & $6.638 \mathrm{e}+00$ & $9.939 \mathrm{e}+00$ & $1.170 \mathrm{e}-02$ & $1.752 \mathrm{e}-02$ \\
\hline 1.2745 & $4.927 e+07$ & $2.357 e+02$ & $3.506 e+02$ & $4.133 e-01$ & $6.149 \mathrm{e}-01$ \\
\hline
\end{tabular}


KBC-40179 rev 1

\begin{tabular}{|c|c|c|c|c|c|}
\hline 1.3325 & $1.630 \mathrm{e}+07$ & $8.247 e+01$ & $1.212 \mathrm{e}+02$ & $1.431 \mathrm{e}-01$ & $2.103 e-01$ \\
\hline 1.3652 & $1.130 \mathrm{e}+06$ & $5.899 \mathrm{e}+00$ & $8.613 e+00$ & $1.017 \mathrm{e}-02$ & $1.485 \mathrm{e}-02$ \\
\hline 1.4944 & $9.017 e+05$ & $5.275 e+00$ & $7.524 \mathrm{e}+00$ & $8.883 e-03$ & $1.267 e-02$ \\
\hline 1.5952 & $3.991 \mathrm{e}+06$ & $2.534 \mathrm{e}+01$ & $3.559 e+01$ & $4.192 \mathrm{e}-02$ & $5.887 \mathrm{e}-02$ \\
\hline Totals & $1.825 \mathrm{e}+10$ & $3.427 e+04$ & $6.296 \mathrm{e}+04$ & $6.651 \mathrm{e}+01$ & $1.226 \mathrm{e}+02$ \\
\hline \multicolumn{6}{|c|}{ Results - Dose Point \# $4-(3.13 \mathrm{e}+02,1.1,0) \mathrm{cm}$} \\
\hline Energy (MeV) & Activity (Photons/sec) & $\begin{array}{l}\text { Fluence Rate } \\
\mathrm{MeV} / \mathrm{cm}^{2} / \mathrm{sec} \\
\text { No Buildup }\end{array}$ & $\begin{array}{l}\text { Fluence Rate } \\
\mathrm{MeV} / \mathrm{cm}^{2} / \mathrm{sec} \\
\text { With Buildup }\end{array}$ & $\begin{array}{c}\text { Exposure Rate } \\
\text { mR/hr } \\
\text { No Buildup }\end{array}$ & $\begin{array}{c}\text { Exposure Rate } \\
\mathrm{mR} / \mathrm{hr} \\
\text { With Buildup }\end{array}$ \\
\hline 0.043 & $1.826 \mathrm{e}+09$ & $1.104 \mathrm{e}+01$ & $4.903 e+01$ & $4.073 e-02$ & $1.809 \mathrm{e}-01$ \\
\hline 0.5923 & $5.491 \mathrm{e}+07$ & $1.131 \mathrm{e}+01$ & $2.224 \mathrm{e}+01$ & $2.209 \mathrm{e}-02$ & $4.343 e-02$ \\
\hline 0.6618 & $1.617 \mathrm{e}+10$ & $3.852 \mathrm{e}+03$ & $7.232 e+03$ & $7.467 \mathrm{e}+00$ & $1.402 \mathrm{e}+01$ \\
\hline 0.7924 & $4.274 \mathrm{e}+07$ & $1.288 \mathrm{e}+01$ & $2.254 \mathrm{e}+01$ & $2.452 \mathrm{e}-02$ & $4.293 e-02$ \\
\hline 0.8759 & $1.775 e+07$ & $6.090 \mathrm{e}+00$ & $1.031 \mathrm{e}+01$ & $1.145 \mathrm{e}-02$ & $1.939 \mathrm{e}-02$ \\
\hline 1.0017 & $3.912 \mathrm{e}+07$ & $1.598 \mathrm{e}+01$ & $2.594 \mathrm{e}+01$ & $2.945 e-02$ & $4.780 \mathrm{e}-02$ \\
\hline 1.0416 & $5.689 e+05$ & $2.444 \mathrm{e}-01$ & $3.921 \mathrm{e}-01$ & $4.473 e-04$ & $7.175 e-04$ \\
\hline 1.1185 & $1.429 \mathrm{e}+05$ & $6.730 \mathrm{e}-02$ & $1.056 \mathrm{e}-01$ & $1.215 \mathrm{e}-04$ & $1.907 e-04$ \\
\hline 1.1509 & $1.339 \mathrm{e}+06$ & $6.545 \mathrm{e}-01$ & $1.018 \mathrm{e}+00$ & $1.174 \mathrm{e}-03$ & $1.827 \mathrm{e}-03$ \\
\hline 1.1732 & $1.630 \mathrm{e}+07$ & $8.162 \mathrm{e}+00$ & $1.263 e+01$ & $1.459 \mathrm{e}-02$ & $2.256 \mathrm{e}-02$ \\
\hline 1.2456 & $1.429 \mathrm{e}+06$ & $7.729 e-01$ & $1.175 e+00$ & $1.363 e-03$ & $2.071 \mathrm{e}-03$ \\
\hline 1.2745 & $4.927 e+07$ & $2.745 e+01$ & $4.143 e+01$ & $4.814 \mathrm{e}-02$ & $7.266 \mathrm{e}-02$ \\
\hline 1.3325 & $1.630 \mathrm{e}+07$ & $9.610 \mathrm{e}+00$ & $1.432 \mathrm{e}+01$ & $1.667 e-02$ & $2.485 \mathrm{e}-02$ \\
\hline 1.3652 & $1.130 \mathrm{e}+06$ & $6.876 \mathrm{e}-01$ & $1.018 \mathrm{e}+00$ & $1.186 \mathrm{e}-03$ & $1.755 e-03$ \\
\hline 1.4944 & $9.017 e+05$ & $6.154 \mathrm{e}-01$ & $8.893 e-01$ & $1.036 \mathrm{e}-03$ & $1.498 \mathrm{e}-03$ \\
\hline 1.5952 & $3.991 \mathrm{e}+06$ & $2.959 \mathrm{e}+00$ & $4.207 e+00$ & $4.895 e-03$ & $6.959 e-03$ \\
\hline Totals & $1.825 \mathrm{e}+10$ & $3.960 \mathrm{e}+03$ & $7.439 e+03$ & $7.685 e+00$ & $1.449 \mathrm{e}+01$ \\
\hline
\end{tabular}


MicroShield 7.02

DOE (7.02-0000)

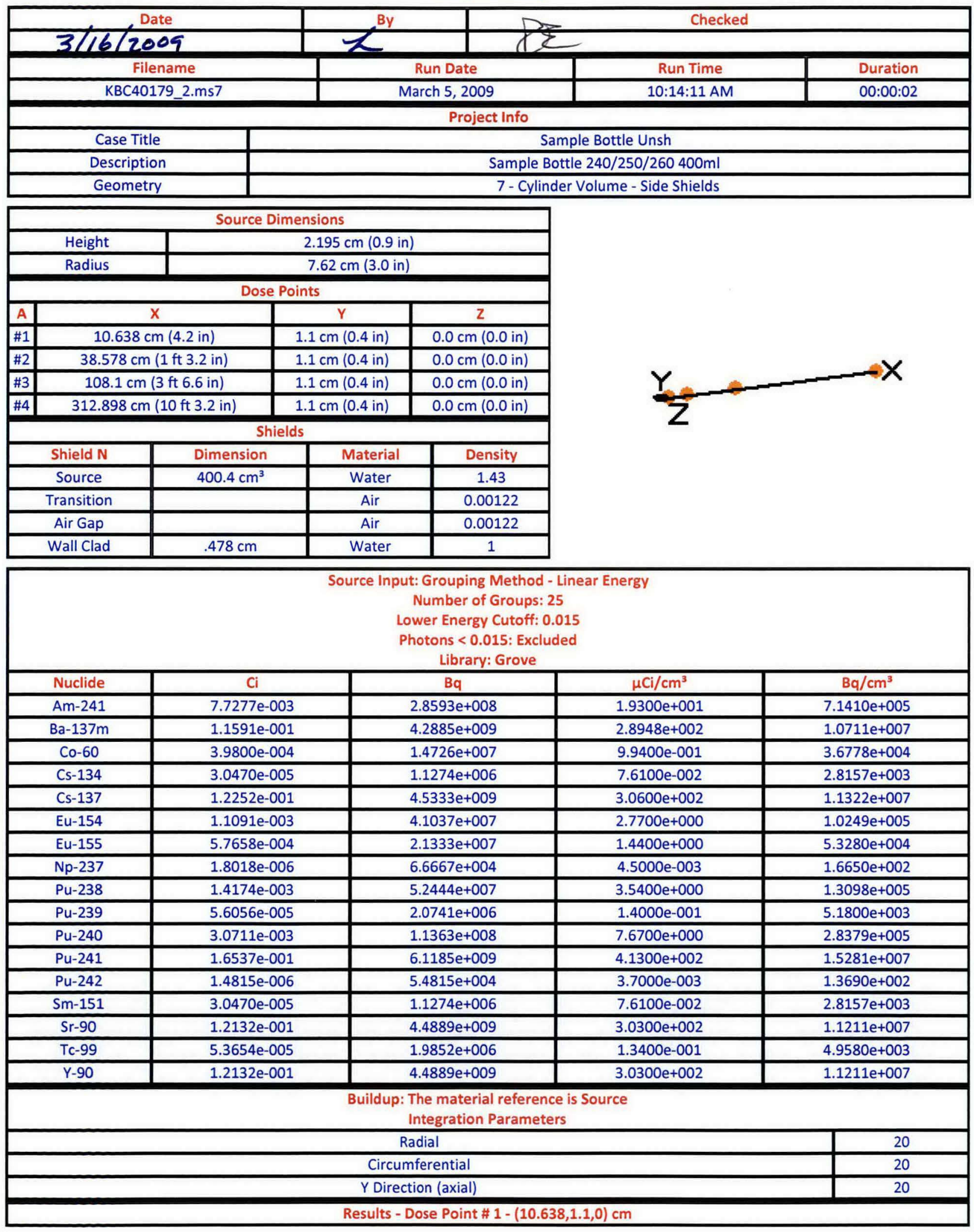




\begin{tabular}{|c|c|c|c|c|c|}
\hline Energy (MeV) & Activity (Photons/sec) & $\begin{array}{l}\text { Fluence Rate } \\
\mathrm{MeV} / \mathrm{cm}^{2} / \mathrm{sec} \\
\text { No Buildup }\end{array}$ & $\begin{array}{l}\text { Fluence Rate } \\
\mathrm{MeV} / \mathrm{cm}^{2} / \mathrm{sec} \\
\text { With Buildup }\end{array}$ & $\begin{array}{c}\text { Exposure Rate } \\
\mathrm{mR} / \mathrm{hr} \\
\text { No Buildup }\end{array}$ & $\begin{array}{c}\text { Exposure Rate } \\
\mathrm{mR} / \mathrm{hr} \\
\text { With Buildup }\end{array}$ \\
\hline 0.045 & $4.691 \mathrm{e}+08$ & $6.461 \mathrm{e}+03$ & $2.249 \mathrm{e}+04$ & $2.146 \mathrm{e}+01$ & $7.470 \mathrm{e}+01$ \\
\hline 0.5816 & $4.112 \mathrm{e}+06$ & $1.324 \mathrm{e}+03$ & $2.118 \mathrm{e}+03$ & $2.589 \mathrm{e}+00$ & $4.142 \mathrm{e}+00$ \\
\hline 0.6618 & $3.869 \mathrm{e}+09$ & $1.456 \mathrm{e}+06$ & $2.241 \mathrm{e}+06$ & $2.822 \mathrm{e}+03$ & $4.344 e+03$ \\
\hline 0.7814 & $3.350 \mathrm{e}+06$ & $1.539 \mathrm{e}+03$ & $2.262 \mathrm{e}+03$ & $2.936 \mathrm{e}+00$ & $4.315 e+00$ \\
\hline 0.8759 & $5.247 e+06$ & $2.765 e+03$ & $3.955 e+03$ & $5.200 \mathrm{e}+00$ & $7.438 \mathrm{e}+00$ \\
\hline 1.0017 & $1.157 \mathrm{e}+07$ & $7.159 e+03$ & $9.948 e+03$ & $1.319 e+01$ & $1.833 e+01$ \\
\hline 1.046 & $6.954 e+04$ & $4.533 e+01$ & $6.241 \mathrm{e}+01$ & $8.289 e-02$ & $1.141 \mathrm{e}-01$ \\
\hline 1.1185 & $4.224 \mathrm{e}+04$ & $2.983 e+01$ & $4.049 \mathrm{e}+01$ & $5.384 e-02$ & $7.308 \mathrm{e}-02$ \\
\hline 1.1372 & $2.184 \mathrm{e}+05$ & $1.573 e+02$ & $2.127 e+02$ & $2.829 \mathrm{e}-01$ & $3.827 e-01$ \\
\hline 1.1732 & $1.473 e+07$ & $1.101 \mathrm{e}+04$ & $1.479 \mathrm{e}+04$ & $1.967 \mathrm{e}+01$ & $2.643 e+01$ \\
\hline 1.2456 & $4.224 \mathrm{e}+05$ & $3.390 \mathrm{e}+02$ & $4.500 e+02$ & $5.978 \mathrm{e}-01$ & $7.934 \mathrm{e}-01$ \\
\hline 1.2745 & $1.457 \mathrm{e}+07$ & $1.201 \mathrm{e}+04$ & $1.587 e+04$ & $2.107 \mathrm{e}+01$ & $2.783 e+01$ \\
\hline 1.3325 & $1.473 e+07$ & $1.280 \mathrm{e}+04$ & $1.676 e+04$ & $2.221 \mathrm{e}+01$ & $2.909 \mathrm{e}+01$ \\
\hline 1.3652 & $3.427 e+04$ & $3.067 \mathrm{e}+01$ & $3.997 \mathrm{e}+01$ & $5.288 \mathrm{e}-02$ & $6.892 \mathrm{e}-02$ \\
\hline 1.4944 & $2.666 \mathrm{e}+05$ & $2.654 \mathrm{e}+02$ & $3.402 \mathrm{e}+02$ & $4.470 \mathrm{e}-01$ & $5.729 \mathrm{e}-01$ \\
\hline 1.5952 & $1.180 \mathrm{e}+06$ & $1.269 \mathrm{e}+03$ & $1.608 \mathrm{e}+03$ & $2.098 \mathrm{e}+00$ & $2.660 \mathrm{e}+00$ \\
\hline Totals & $4.408 \mathrm{e}+09$ & $1.513 e+06$ & $2.332 \mathrm{e}+06$ & $2.934 \mathrm{e}+03$ & $4.541 \mathrm{e}+03$ \\
\hline \multicolumn{6}{|c|}{ Results - Dose Point \# $2-(38.578,1.1,0) \mathrm{cm}$} \\
\hline Energy (MeV) & Activity (Photons/sec) & $\begin{array}{c}\text { Fluence Rate } \\
\mathrm{MeV} / \mathrm{cm}^{2} / \mathrm{sec} \\
\text { No Buildup }\end{array}$ & $\begin{array}{l}\text { Fluence Rate } \\
\mathrm{MeV} / \mathrm{cm}^{2} / \mathrm{sec} \\
\text { With Buildup }\end{array}$ & $\begin{array}{c}\text { Exposure Rate } \\
\mathrm{mR} / \mathrm{hr} \\
\text { No Buildup }\end{array}$ & $\begin{array}{c}\text { Exposure Rate } \\
\mathrm{mR} / \mathrm{hr} \\
\text { With Buildup }\end{array}$ \\
\hline 0.045 & $4.691 \mathrm{e}+08$ & $2.639 \mathrm{e}+02$ & $1.108 \mathrm{e}+03$ & $8.765 \mathrm{e}-01$ & $3.680 \mathrm{e}+00$ \\
\hline 0.5816 & $4.112 \mathrm{e}+06$ & $6.328 \mathrm{e}+01$ & $1.143 e+02$ & $1.237 e-01$ & $2.235 \mathrm{e}-01$ \\
\hline 0.6618 & $3.869 \mathrm{e}+09$ & $7.009 e+04$ & $1.207 e+05$ & $1.359 \mathrm{e}+02$ & $2.340 \mathrm{e}+02$ \\
\hline 0.7814 & $3.350 \mathrm{e}+06$ & $7.482 e+01$ & $1.217 e+02$ & $1.427 e-01$ & $2.321 \mathrm{e}-01$ \\
\hline 0.8759 & $5.247 e+06$ & $1.353 e+02$ & $2.127 e+02$ & $2.544 \mathrm{e}-01$ & $4.000 \mathrm{e}-01$ \\
\hline 1.0017 & $1.157 \mathrm{e}+07$ & $3.529 e+02$ & $5.349 e+02$ & $6.503 e-01$ & $9.857 e-01$ \\
\hline 1.046 & $6.954 e+04$ & $2.240 \mathrm{e}+00$ & $3.356 e+00$ & $4.095 e-03$ & $6.137 e-03$ \\
\hline 1.1185 & $4.224 e+04$ & $1.479 \mathrm{e}+00$ & $2.177 e+00$ & $2.670 \mathrm{e}-03$ & $3.930 \mathrm{e}-03$ \\
\hline 1.1372 & $2.184 \mathrm{e}+05$ & $7.806 \mathrm{e}+00$ & $1.144 \mathrm{e}+01$ & $1.404 \mathrm{e}-02$ & $2.058 \mathrm{e}-02$ \\
\hline 1.1732 & $1.473 e+07$ & $5.472 \mathrm{e}+02$ & $7.953 e+02$ & $9.779 \mathrm{e}-01$ & $1.421 \mathrm{e}+00$ \\
\hline 1.2456 & $4.224 e+05$ & $1.691 \mathrm{e}+01$ & $2.420 \mathrm{e}+01$ & $2.981 \mathrm{e}-02$ & $4.266 \mathrm{e}-02$ \\
\hline 1.2745 & $1.457 \mathrm{e}+07$ & $5.999 e+02$ & $8.534 \mathrm{e}+02$ & $1.052 \mathrm{e}+00$ & $1.497 e+00$ \\
\hline 1.3325 & $1.473 e+07$ & $6.408 \mathrm{e}+02$ & $9.016 e+02$ & $1.112 \mathrm{e}+00$ & $1.564 \mathrm{e}+00$ \\
\hline 1.3652 & $3.427 \mathrm{e}+04$ & $1.537 \mathrm{e}+00$ & $2.149 \mathrm{e}+00$ & $2.650 e-03$ & $3.706 \mathrm{e}-03$ \\
\hline 1.4944 & $2.666 e+05$ & $1.336 \mathrm{e}+01$ & $1.830 \mathrm{e}+01$ & $2.250 \mathrm{e}-02$ & $3.082 \mathrm{e}-02$ \\
\hline 1.5952 & $1.180 \mathrm{e}+06$ & $6.408 \mathrm{e}+01$ & $8.653 e+01$ & $1.060 \mathrm{e}-01$ & $1.431 \mathrm{e}-01$ \\
\hline Totals & $4.408 \mathrm{e}+09$ & $7.287 e+04$ & $1.255 e+05$ & $1.412 \mathrm{e}+02$ & $2.443 e+02$ \\
\hline \multicolumn{6}{|c|}{ Results - Dose Point \# 3 - $(108.1,1.1,0) \mathrm{cm}$} \\
\hline Energy (MeV) & Activity (Photons/sec) & $\begin{array}{c}\text { Fluence Rate } \\
\mathrm{MeV} / \mathrm{cm}^{2} / \mathrm{sec} \\
\text { No Buildup }\end{array}$ & $\begin{array}{l}\text { Fluence Rate } \\
\mathrm{MeV} / \mathrm{cm}^{2} / \mathrm{sec} \\
\text { With Buildup }\end{array}$ & $\begin{array}{c}\text { Exposure Rate } \\
\mathrm{mR} / \mathrm{hr} \\
\text { No Buildup }\end{array}$ & $\begin{array}{c}\text { Exposure Rate } \\
\mathrm{mR} / \mathrm{hr} \\
\text { With Buildup }\end{array}$ \\
\hline 0.045 & $4.691 \mathrm{e}+08$ & $3.060 \mathrm{e}+01$ & $1.344 \mathrm{e}+02$ & $1.016 \mathrm{e}-01$ & $4.465 \mathrm{e}-01$ \\
\hline 0.5816 & $4.112 \mathrm{e}+06$ & $7.639 e+00$ & $1.419 \mathrm{e}+01$ & $1.494 \mathrm{e}-02$ & $2.775 \mathrm{e}-02$ \\
\hline 0.6618 & $3.869 \mathrm{e}+09$ & $8.477 e+03$ & $1.498 \mathrm{e}+04$ & $1.643 e+01$ & $2.905 \mathrm{e}+01$ \\
\hline 0.7814 & $3.350 \mathrm{e}+06$ & $9.070 \mathrm{e}+00$ & $1.510 \mathrm{e}+01$ & $1.730 \mathrm{e}-02$ & $2.880 \mathrm{e}-02$ \\
\hline 0.8759 & $5.247 e+06$ & $1.643 e+01$ & $2.639 e+01$ & $3.089 \mathrm{e}-02$ & $4.963 e-02$ \\
\hline 1.0017 & $1.157 e+07$ & $4.292 \mathrm{e}+01$ & $6.637 e+01$ & $7.909 \mathrm{e}-02$ & $1.223 \mathrm{e}-01$ \\
\hline 1.046 & $6.954 e+04$ & $2.725 \mathrm{e}-01$ & $4.164 \mathrm{e}-01$ & $4.984 \mathrm{e}-04$ & $7.615 e-04$ \\
\hline 1.1185 & $4.224 e+04$ & $1.802 \mathrm{e}-01$ & $2.701 \mathrm{e}-01$ & $3.252 \mathrm{e}-04$ & $4.876 e-04$ \\
\hline 1.1372 & $2.184 \mathrm{e}+05$ & $9.510 \mathrm{e}-01$ & $1.419 \mathrm{e}+00$ & $1.711 \mathrm{e}-03$ & $2.553 e-03$ \\
\hline 1.1732 & $1.473 e+07$ & $6.669 \mathrm{e}+01$ & $9.869 e+01$ & $1.192 \mathrm{e}-01$ & $1.764 \mathrm{e}-01$ \\
\hline 1.2456 & $4.224 \mathrm{e}+05$ & $2.062 \mathrm{e}+00$ & $3.003 e+00$ & $3.636 \mathrm{e}-03$ & $5.294 \mathrm{e}-03$ \\
\hline 1.2745 & $1.457 e+07$ & $7.319 e+01$ & $1.059 \mathrm{e}+02$ & $1.283 \mathrm{e}-01$ & $1.857 \mathrm{e}-01$ \\
\hline
\end{tabular}




\begin{tabular}{|c|c|c|c|c|c|}
\hline 1.3325 & $1.473 e+07$ & $7.822 \mathrm{e}+01$ & $1.119 \mathrm{e}+02$ & $1.357 \mathrm{e}-01$ & $1.941 \mathrm{e}-01$ \\
\hline 1.3652 & $3.427 e+04$ & $1.876 \mathrm{e}-01$ & $2.667 e-01$ & $3.236 \mathrm{e}-04$ & $4.600 \mathrm{e}-04$ \\
\hline 1.4944 & $2.666 \mathrm{e}+05$ & $1.633 e+00$ & $2.271 \mathrm{e}+00$ & $2.751 \mathrm{e}-03$ & $3.825 \mathrm{e}-03$ \\
\hline 1.5952 & $1.180 \mathrm{e}+06$ & $7.838 \mathrm{e}+00$ & $1.074 \mathrm{e}+01$ & $1.297 e-02$ & $1.776 \mathrm{e}-02$ \\
\hline Totals & $4.408 \mathrm{e}+09$ & $8.815 \mathrm{e}+03$ & $1.558 \mathrm{e}+04$ & $1.708 \mathrm{e}+01$ & $3.031 \mathrm{e}+01$ \\
\hline \multicolumn{6}{|c|}{ Results - Dose Point \# 4 - $(312.898,1.1,0) \mathrm{cm}$} \\
\hline Energy (MeV) & Activity (Photons/sec) & $\begin{array}{c}\text { Fluence Rate } \\
\mathrm{MeV} / \mathrm{cm}^{2} / \mathrm{sec} \\
\text { No Buildup }\end{array}$ & $\begin{array}{l}\text { Fluence Rate } \\
\mathrm{MeV} / \mathrm{cm}^{2} / \mathrm{sec} \\
\text { With Buildup }\end{array}$ & $\begin{array}{c}\text { Exposure Rate } \\
\text { mR/hr } \\
\text { No Buildup }\end{array}$ & $\begin{array}{c}\text { Exposure Rate } \\
\mathrm{mR} / \mathrm{hr} \\
\text { With Buildup }\end{array}$ \\
\hline 0.045 & $4.691 \mathrm{e}+08$ & $3.386 \mathrm{e}+00$ & $1.567 e+01$ & $1.125 \mathrm{e}-02$ & $5.203 \mathrm{e}-02$ \\
\hline 0.5816 & $4.112 \mathrm{e}+06$ & $8.822 \mathrm{e}-01$ & $1.679 \mathrm{e}+00$ & $1.725 \mathrm{e}-03$ & $3.282 \mathrm{e}-03$ \\
\hline 0.6618 & $3.869 \mathrm{e}+09$ & $9.805 e+02$ & $1.772 \mathrm{e}+03$ & $1.901 \mathrm{e}+00$ & $3.435 \mathrm{e}+00$ \\
\hline 0.7814 & $3.350 \mathrm{e}+06$ & $1.051 \mathrm{e}+00$ & $1.785 e+00$ & $2.005 e-03$ & $3.405 e-03$ \\
\hline 0.8759 & $5.247 e+06$ & $1.906 \mathrm{e}+00$ & $3.120 \mathrm{e}+00$ & $3.585 \mathrm{e}-03$ & $5.867 \mathrm{e}-03$ \\
\hline 1.0017 & $1.157 e+07$ & $4.989 \mathrm{e}+00$ & $7.847 e+00$ & $9.194 \mathrm{e}-03$ & $1.446 \mathrm{e}-02$ \\
\hline 1.046 & $6.954 \mathrm{e}+04$ & $3.170 \mathrm{e}-02$ & $4.923 e-02$ & $5.796 e-05$ & $9.003 e-05$ \\
\hline 1.1185 & $4.224 \mathrm{e}+04$ & $2.097 e-02$ & $3.194 \mathrm{e}-02$ & $3.785 e-05$ & $5.765 e-05$ \\
\hline 1.1372 & $2.184 \mathrm{e}+05$ & $1.107 \mathrm{e}-01$ & $1.678 \mathrm{e}-01$ & $1.992 \mathrm{e}-04$ & $3.019 \mathrm{e}-04$ \\
\hline 1.1732 & $1.473 e+07$ & $7.766 \mathrm{e}+00$ & $1.167 e+01$ & $1.388 \mathrm{e}-02$ & $2.085 e-02$ \\
\hline 1.2456 & $4.224 e+05$ & $2.403 e-01$ & $3.550 \mathrm{e}-01$ & $4.237 e-04$ & $6.260 \mathrm{e}-04$ \\
\hline 1.2745 & $1.457 \mathrm{e}+07$ & $8.531 \mathrm{e}+00$ & $1.252 \mathrm{e}+01$ & $1.496 \mathrm{e}-02$ & $2.196 \mathrm{e}-02$ \\
\hline 1.3325 & $1.473 e+07$ & $9.122 \mathrm{e}+00$ & $1.323 e+01$ & $1.583 e-02$ & $2.295 \mathrm{e}-02$ \\
\hline 1.3652 & $3.427 e+04$ & $2.189 \mathrm{e}-02$ & $3.154 \mathrm{e}-02$ & $3.775 e-05$ & $5.439 e-05$ \\
\hline 1.4944 & $2.666 e+05$ & $1.907 \mathrm{e}-01$ & $2.685 e-01$ & $3.212 \mathrm{e}-04$ & $4.523 e-04$ \\
\hline 1.5952 & $1.180 \mathrm{e}+06$ & $9.158 \mathrm{e}-01$ & $1.270 \mathrm{e}+00$ & $1.515 e-03$ & $2.101 \mathrm{e}-03$ \\
\hline Totals & $4.408 \mathrm{e}+09$ & $1.020 \mathrm{e}+03$ & $1.842 \mathrm{e}+03$ & $1.976 \mathrm{e}+00$ & $3.584 e+00$ \\
\hline
\end{tabular}




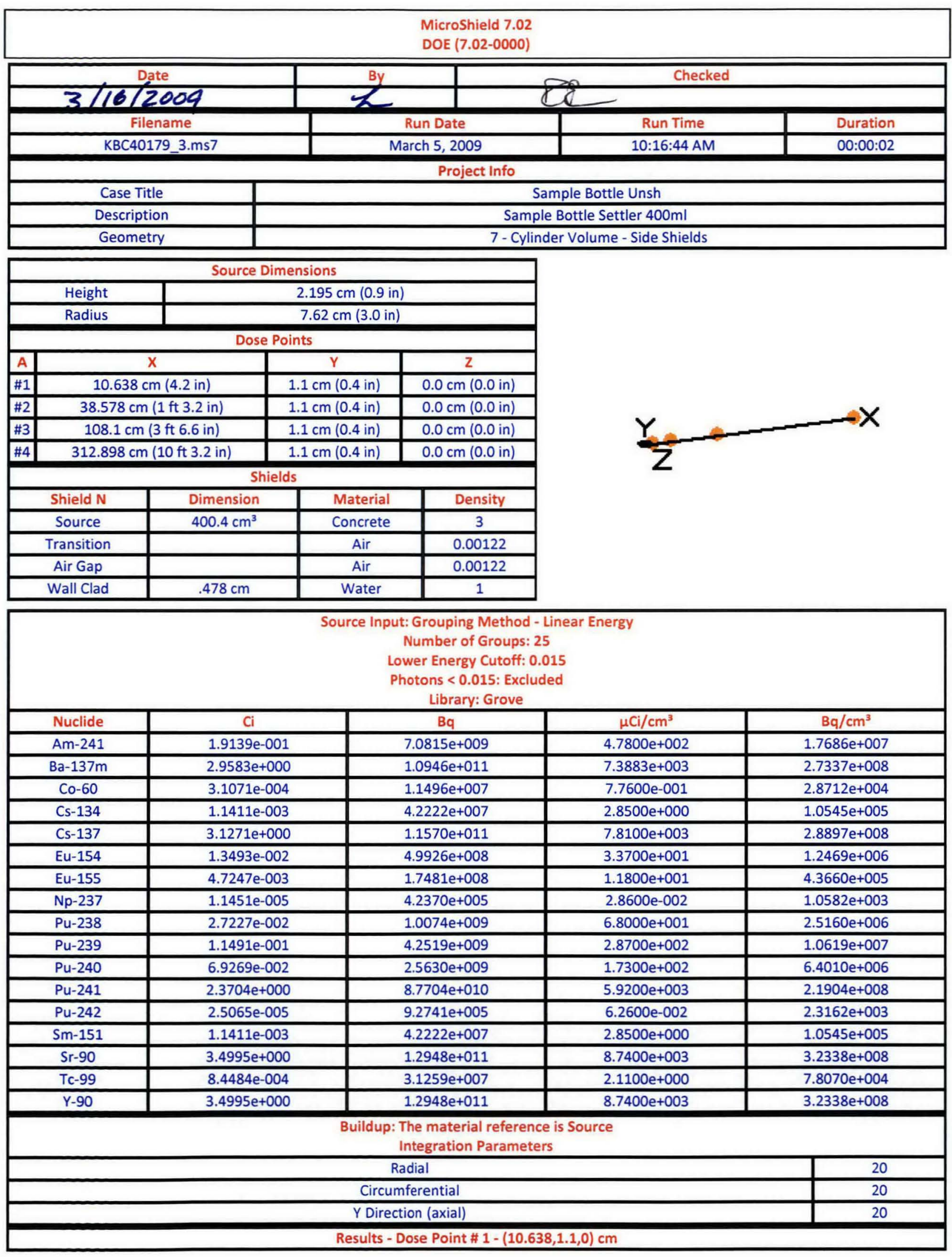




\begin{tabular}{|c|c|c|c|c|c|}
\hline Energy (MeV) & Activity (Photons/sec) & $\begin{array}{c}\text { Fluence Rate } \\
\mathrm{MeV} / \mathrm{cm}^{2} / \mathrm{sec} \\
\text { No Buildup }\end{array}$ & $\begin{array}{l}\text { Fluence Rate } \\
\mathrm{MeV} / \mathrm{cm}^{2} / \mathrm{sec} \\
\text { With Buildup }\end{array}$ & $\begin{array}{c}\text { Exposure Rate } \\
\text { mR/hr } \\
\text { No Buildup }\end{array}$ & $\begin{array}{c}\text { Exposure Rate } \\
\mathrm{mR} / \mathrm{hr} \\
\text { With Buildup }\end{array}$ \\
\hline 0.0419 & $1.120 \mathrm{e}+10$ & $4.190 \mathrm{e}+04$ & $6.121 \mathrm{e}+04$ & $1.652 \mathrm{e}+02$ & $2.414 \mathrm{e}+02$ \\
\hline 0.5875 & $8.505 e+07$ & $2.005 e+04$ & $3.587 e+04$ & $3.918 \mathrm{e}+01$ & $7.010 \mathrm{e}+01$ \\
\hline 0.6617 & $9.861 e+10$ & $2.708 \mathrm{e}+07$ & $4.681 \mathrm{e}+07$ & $5.249 \mathrm{e}+04$ & $9.075 e+04$ \\
\hline 0.7873 & $6.758 \mathrm{e}+07$ & $2.320 \mathrm{e}+04$ & $3.827 e+04$ & $4.420 \mathrm{e}+01$ & $7.293 e+01$ \\
\hline 0.8759 & $6.383 e+07$ & $2.512 \mathrm{e}+04$ & $4.034 \mathrm{e}+04$ & $4.723 e+01$ & $7.587 e+01$ \\
\hline 1.0017 & $1.407 e+08$ & $6.576 e+04$ & $1.022 e+05$ & $1.212 \mathrm{e}+02$ & $1.883 e+02$ \\
\hline 1.0441 & $1.131 \mathrm{e}+06$ & $5.574 e+02$ & $8.576 e+02$ & $1.020 \mathrm{e}+00$ & $1.569 \mathrm{e}+00$ \\
\hline 1.1185 & $5.139 e+05$ & $2.766 \mathrm{e}+02$ & $4.184 \mathrm{e}+02$ & $4.992 \mathrm{e}-01$ & $7.553 e-01$ \\
\hline 1.1421 & $3.170 \mathrm{e}+06$ & $1.752 \mathrm{e}+03$ & $2.638 \mathrm{e}+03$ & $3.149 \mathrm{e}+00$ & $4.740 \mathrm{e}+00$ \\
\hline 1.1732 & $1.150 \mathrm{e}+07$ & $6.575 e+03$ & $9.835 e+03$ & $1.175 \mathrm{e}+01$ & $1.758 \mathrm{e}+01$ \\
\hline 1.2456 & $5.139 \mathrm{e}+06$ & $3.173 e+03$ & $4.679 e+03$ & $5.594 e+00$ & $8.249 e+00$ \\
\hline 1.2745 & $1.772 \mathrm{e}+08$ & $1.126 e+05$ & $1.652 e+05$ & $1.975 \mathrm{e}+02$ & $2.898 \mathrm{e}+02$ \\
\hline 1.3325 & $1.150 \mathrm{e}+07$ & $7.733 e+03$ & $1.123 e+04$ & $1.342 \mathrm{e}+01$ & $1.948 \mathrm{e}+01$ \\
\hline 1.3652 & $1.284 \mathrm{e}+06$ & $8.903 e+02$ & $1.286 \mathrm{e}+03$ & $1.535 \mathrm{e}+00$ & $2.218 \mathrm{e}+00$ \\
\hline 1.4944 & $3.243 e+06$ & $2.522 \mathrm{e}+03$ & $3.574 \mathrm{e}+03$ & $4.248 \mathrm{e}+00$ & $6.019 e+00$ \\
\hline 1.5952 & $1.435 e+07$ & $1.212 \mathrm{e}+04$ & $1.696 \mathrm{e}+04$ & $2.005 e+01$ & $2.805 e+01$ \\
\hline Totals & $1.104 \mathrm{e}+11$ & $2.740 \mathrm{e}+07$ & $4.730 \mathrm{e}+07$ & $5.317 e+04$ & $9.177 e+04$ \\
\hline \multicolumn{6}{|c|}{ Results - Dose Point \# 2 - $(38.578,1.1,0) \mathrm{cm}$} \\
\hline Energy (MeV) & Activity (Photons/sec) & $\begin{array}{c}\text { Fluence Rate } \\
\mathrm{MeV} / \mathrm{cm}^{2} / \mathrm{sec} \\
\text { No Buildup }\end{array}$ & $\begin{array}{l}\text { Fluence Rate } \\
\mathrm{MeV} / \mathrm{cm}^{2} / \mathrm{sec} \\
\text { With Buildup }\end{array}$ & $\begin{array}{c}\text { Exposure Rate } \\
\text { mR/hr } \\
\text { No Buildup } \\
\end{array}$ & $\begin{array}{c}\text { Exposure Rate } \\
\mathrm{mR} / \mathrm{hr} \\
\text { With Buildup }\end{array}$ \\
\hline 0.0419 & $1.120 \mathrm{e}+10$ & $1.358 \mathrm{e}+03$ & $2.024 \mathrm{e}+03$ & $5.354 \mathrm{e}+00$ & $7.981 \mathrm{e}+00$ \\
\hline 0.5875 & $8.505 e+07$ & $8.667 e+02$ & $1.781 \mathrm{e}+03$ & $1.694 \mathrm{e}+00$ & $3.480 \mathrm{e}+00$ \\
\hline 0.6617 & $9.861 \mathrm{e}+10$ & $1.182 \mathrm{e}+06$ & $2.332 e+06$ & $2.291 \mathrm{e}+03$ & $4.522 e+03$ \\
\hline 0.7873 & $6.758 \mathrm{e}+07$ & $1.026 \mathrm{e}+03$ & $1.917 e+03$ & $1.956 \mathrm{e}+00$ & $3.653 e+00$ \\
\hline 0.8759 & $6.383 e+07$ & $1.121 \mathrm{e}+03$ & $2.027 e+03$ & $2.108 \mathrm{e}+00$ & $3.813 e+00$ \\
\hline 1.0017 & $1.407 \mathrm{e}+08$ & $2.967 e+03$ & $5.156 e+03$ & $5.468 \mathrm{e}+00$ & $9.502 e+00$ \\
\hline 1.0441 & $1.131 \mathrm{e}+06$ & $2.524 \mathrm{e}+01$ & $4.332 e+01$ & $4.616 \mathrm{e}-02$ & $7.925 \mathrm{e}-02$ \\
\hline 1.1185 & $5.139 e+05$ & $1.259 \mathrm{e}+01$ & $2.118 \mathrm{e}+01$ & $2.273 e-02$ & $3.824 \mathrm{e}-02$ \\
\hline 1.1421 & $3.170 \mathrm{e}+06$ & $7.990 \mathrm{e}+01$ & $1.336 \mathrm{e}+02$ & $1.436 \mathrm{e}-01$ & $2.401 \mathrm{e}-01$ \\
\hline 1.1732 & $1.150 \mathrm{e}+07$ & $3.005 e+02$ & $4.986 \mathrm{e}+02$ & $5.370 \mathrm{e}-01$ & $8.910 \mathrm{e}-01$ \\
\hline 1.2456 & $5.139 e+06$ & $1.457 e+02$ & $2.376 \mathrm{e}+02$ & $2.569 \mathrm{e}-01$ & $4.190 \mathrm{e}-01$ \\
\hline 1.2745 & $1.772 \mathrm{e}+08$ & $5.182 \mathrm{e}+03$ & $8.398 \mathrm{e}+03$ & $9.089 e+00$ & $1.473 e+01$ \\
\hline 1.3325 & $1.150 \mathrm{e}+07$ & $3.570 \mathrm{e}+02$ & $5.715 e+02$ & $6.194 \mathrm{e}-01$ & $9.915 e-01$ \\
\hline 1.3652 & $1.284 \mathrm{e}+06$ & $4.119 e+01$ & $6.550 \mathrm{e}+01$ & $7.102 e-02$ & $1.129 \mathrm{e}-01$ \\
\hline 1.4944 & $3.243 e+06$ & $1.175 e+02$ & $1.825 e+02$ & $1.979 \mathrm{e}-01$ & $3.074 \mathrm{e}-01$ \\
\hline 1.5952 & $1.435 \mathrm{e}+07$ & $5.675 e+02$ & $8.677 e+02$ & $9.387 e-01$ & $1.435 \mathrm{e}+00$ \\
\hline Totals & $1.104 \mathrm{e}+11$ & $1.196 \mathrm{e}+06$ & $2.356 \mathrm{e}+06$ & $2.319 \mathrm{e}+03$ & $4.569 e+03$ \\
\hline \multicolumn{6}{|c|}{ Results - Dose Point \# 3 - $(108.1,1.1,0) \mathrm{cm}$} \\
\hline Energy (MeV) & Activity (Photons/sec) & $\begin{array}{c}\text { Fluence Rate } \\
\mathrm{MeV} / \mathrm{cm}^{2} / \mathrm{sec} \\
\text { No Buildup }\end{array}$ & $\begin{array}{l}\text { Fluence Rate } \\
\mathrm{MeV} / \mathrm{cm}^{2} / \mathrm{sec} \\
\text { With Buildup }\end{array}$ & $\begin{array}{c}\text { Exposure Rate } \\
\mathrm{mR} / \mathrm{hr} \\
\text { No Buildup }\end{array}$ & $\begin{array}{c}\text { Exposure Rate } \\
\mathrm{mR} / \mathrm{hr} \\
\text { With Buildup }\end{array}$ \\
\hline 0.0419 & $1.120 \mathrm{e}+10$ & $1.509 \mathrm{e}+02$ & $2.269 \mathrm{e}+02$ & $5.949 \mathrm{e}-01$ & $8.946 \mathrm{e}-01$ \\
\hline 0.5875 & $8.505 e+07$ & $1.027 \mathrm{e}+02$ & $2.172 \mathrm{e}+02$ & $2.006 \mathrm{e}-01$ & $4.244 \mathrm{e}-01$ \\
\hline 0.6617 & $9.861 \mathrm{e}+10$ & $1.403 e+05$ & $2.847 e+05$ & $2.719 e+02$ & $5.520 \mathrm{e}+02$ \\
\hline 0.7873 & $6.758 \mathrm{e}+07$ & $1.222 \mathrm{e}+02$ & $2.344 \mathrm{e}+02$ & $2.329 \mathrm{e}-01$ & $4.466 \mathrm{e}-01$ \\
\hline 0.8759 & $6.383 e+07$ & $1.338 \mathrm{e}+02$ & $2.480 \mathrm{e}+02$ & $2.515 e-01$ & $4.664 \mathrm{e}-01$ \\
\hline 1.0017 & $1.407 e+08$ & $3.549 \mathrm{e}+02$ & $6.314 \mathrm{e}+02$ & $6.539 \mathrm{e}-01$ & $1.163 e+00$ \\
\hline 1.0441 & $1.131 \mathrm{e}+06$ & $3.020 \mathrm{e}+00$ & $5.306 e+00$ & $5.525 e-03$ & $9.706 \mathrm{e}-03$ \\
\hline 1.1185 & $5.139 \mathrm{e}+05$ & $1.509 \mathrm{e}+00$ & $2.596 \mathrm{e}+00$ & $2.724 \mathrm{e}-03$ & $4.686 \mathrm{e}-03$ \\
\hline 1.1421 & $3.170 \mathrm{e}+06$ & $9.579 \mathrm{e}+00$ & $1.637 e+01$ & $1.722 \mathrm{e}-02$ & $2.943 e-02$ \\
\hline 1.1732 & $1.150 \mathrm{e}+07$ & $3.604 \mathrm{e}+01$ & $6.112 e+01$ & $6.441 \mathrm{e}-02$ & $1.092 \mathrm{e}-01$ \\
\hline 1.2456 & $5.139 e+06$ & $1.749 \mathrm{e}+01$ & $2.914 \mathrm{e}+01$ & $3.084 \mathrm{e}-02$ & $5.138 \mathrm{e}-02$ \\
\hline 1.2745 & $1.772 \mathrm{e}+08$ & $6.225 e+02$ & $1.030 \mathrm{e}+03$ & $1.092 \mathrm{e}+00$ & $1.806 \mathrm{e}+00$ \\
\hline
\end{tabular}


KBC-40179 rev 1

\begin{tabular}{|c|c|c|c|c|c|}
\hline 1.3325 & $1.150 \mathrm{e}+07$ & $4.292 e+01$ & $7.012 \mathrm{e}+01$ & $7.446 \mathrm{e}-02$ & $1.217 e-01$ \\
\hline 1.3652 & $1.284 \mathrm{e}+06$ & $4.953 e+00$ & $8.037 e+00$ & $8.541 \mathrm{e}-03$ & $1.386 \mathrm{e}-02$ \\
\hline 1.4944 & $3.243 e+06$ & $1.415 \mathrm{e}+01$ & $2.241 \mathrm{e}+01$ & $2.383 e-02$ & $3.775 e-02$ \\
\hline 1.5952 & $1.435 e+07$ & $6.843 e+01$ & $1.066 \mathrm{e}+02$ & $1.132 \mathrm{e}-01$ & $1.763 e-01$ \\
\hline Totals & $1.104 \mathrm{e}+11$ & $1.420 \mathrm{e}+05$ & $2.876 \mathrm{e}+05$ & $2.753 e+02$ & $5.577 e+02$ \\
\hline \multicolumn{6}{|c|}{ Results - Dose Point \# 4 - $(312.898,1.1,0) \mathrm{cm}$} \\
\hline Energy (MeV) & Activity (Photons/sec) & $\begin{array}{l}\text { Fluence Rate } \\
\mathrm{MeV} / \mathrm{cm}^{2} / \mathrm{sec} \\
\text { No Buildup }\end{array}$ & $\begin{array}{l}\text { Fluence Rate } \\
\mathrm{MeV} / \mathrm{cm}^{2} / \mathrm{sec} \\
\text { With Buildup }\end{array}$ & $\begin{array}{c}\text { Exposure Rate } \\
\mathrm{mR} / \mathrm{hr} \\
\text { No Buildup }\end{array}$ & $\begin{array}{c}\text { Exposure Rate } \\
\mathrm{mR} / \mathrm{hr} \\
\text { With Buildup }\end{array}$ \\
\hline 0.0419 & $1.120 \mathrm{e}+10$ & $1.647 \mathrm{e}+01$ & $2.517 \mathrm{e}+01$ & $6.496 \mathrm{e}-02$ & $9.925 \mathrm{e}-02$ \\
\hline 0.5875 & $8.505 e+07$ & $1.178 \mathrm{e}+01$ & $2.546 \mathrm{e}+01$ & $2.302 \mathrm{e}-02$ & $4.975 \mathrm{e}-02$ \\
\hline 0.6617 & $9.861 e+10$ & $1.612 e+04$ & $3.339 e+04$ & $3.125 e+01$ & $6.473 e+01$ \\
\hline 0.7873 & $6.758 \mathrm{e}+07$ & $1.408 \mathrm{e}+01$ & $2.750 \mathrm{e}+01$ & $2.683 e-02$ & $5.241 \mathrm{e}-02$ \\
\hline 0.8759 & $6.383 e+07$ & $1.543 e+01$ & $2.912 \mathrm{e}+01$ & $2.902 \mathrm{e}-02$ & $5.476 \mathrm{e}-02$ \\
\hline 1.0017 & $1.407 e+08$ & $4.101 \mathrm{e}+01$ & $7.417 e+01$ & $7.557 \mathrm{e}-02$ & $1.367 \mathrm{e}-01$ \\
\hline 1.0441 & $1.131 \mathrm{e}+06$ & $3.492 \mathrm{e}-01$ & $6.235 \mathrm{e}-01$ & $6.388 \mathrm{e}-04$ & $1.140 \mathrm{e}-03$ \\
\hline 1.1185 & $5.139 e+05$ & $1.746 \mathrm{e}-01$ & $3.051 \mathrm{e}-01$ & $3.152 \mathrm{e}-04$ & $5.507 e-04$ \\
\hline 1.1421 & $3.170 \mathrm{e}+06$ & $1.109 \mathrm{e}+00$ & $1.925 \mathrm{e}+00$ & $1.993 e-03$ & $3.459 \mathrm{e}-03$ \\
\hline 1.1732 & $1.150 \mathrm{e}+07$ & $4.174 e+00$ & $7.185 e+00$ & $7.459 \mathrm{e}-03$ & $1.284 \mathrm{e}-02$ \\
\hline 1.2456 & $5.139 e+06$ & $2.027 e+00$ & $3.427 e+00$ & $3.574 \mathrm{e}-03$ & $6.042 \mathrm{e}-03$ \\
\hline 1.2745 & $1.772 e+08$ & $7.216 e+01$ & $1.211 \mathrm{e}+02$ & $1.265 \mathrm{e}-01$ & $2.124 \mathrm{e}-01$ \\
\hline 1.3325 & $1.150 \mathrm{e}+07$ & $4.978 \mathrm{e}+00$ & $8.247 e+00$ & $8.636 e-03$ & $1.431 \mathrm{e}-02$ \\
\hline 1.3652 & $1.284 e+06$ & $5.746 e-01$ & $9.454 \mathrm{e}-01$ & $9.909 \mathrm{e}-04$ & $1.630 \mathrm{e}-03$ \\
\hline 1.4944 & $3.243 e+06$ & $1.644 \mathrm{e}+00$ & $2.637 e+00$ & $2.768 \mathrm{e}-03$ & $4.442 \mathrm{e}-03$ \\
\hline 1.5952 & $1.435 e+07$ & $7.953 e+00$ & $1.255 e+01$ & $1.316 \mathrm{e}-02$ & $2.075 e-02$ \\
\hline Totals & $1.104 \mathrm{e}+11$ & $1.631 e+04$ & $3.373 e+04$ & $3.164 \mathrm{e}+01$ & $6.540 e+01$ \\
\hline
\end{tabular}




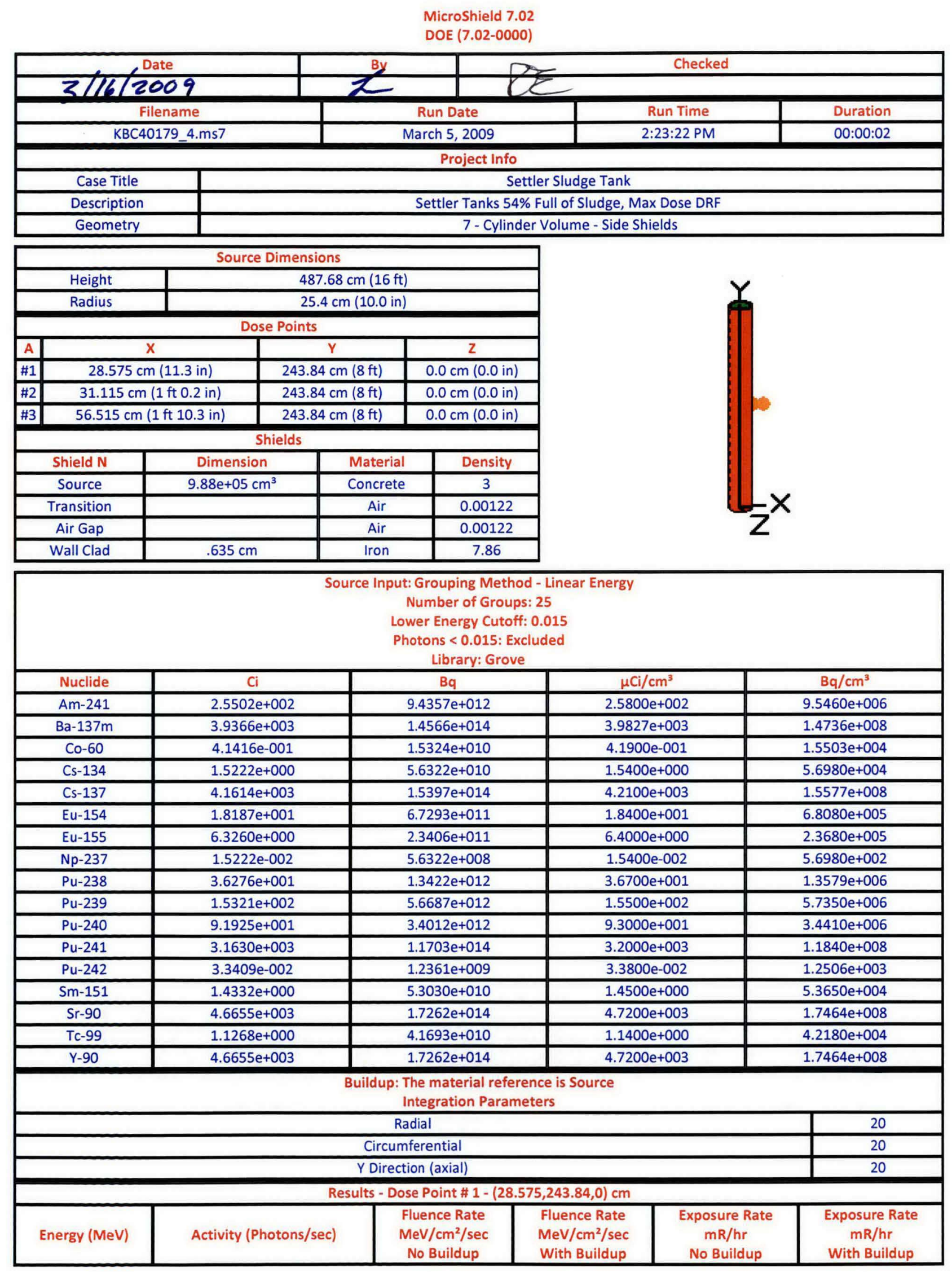




\begin{tabular}{|c|c|c|c|c|c|}
\hline 0.0419 & $1.492 \mathrm{e}+13$ & $1.208 \mathrm{e}-04$ & $4.623 e-04$ & $4.754 \mathrm{e}-07$ & $1.819 \mathrm{e}-06$ \\
\hline 0.5874 & $1.139 \mathrm{e}+11$ & $4.857 \mathrm{e}+04$ & $1.608 \mathrm{e}+05$ & $9.492 e+01$ & $3.143 e+02$ \\
\hline 0.6617 & $1.312 \mathrm{e}+14$ & $6.858 \mathrm{e}+07$ & $2.153 e+08$ & $1.329 \mathrm{e}+05$ & $4.173 e+05$ \\
\hline 0.7873 & $9.054 e+10$ & $6.369 e+04$ & $1.857 e+05$ & $1.214 e+02$ & $3.538 \mathrm{e}+02$ \\
\hline 0.8759 & $8.603 e+10$ & $7.266 e+04$ & $2.027 e+05$ & $1.366 e+02$ & $3.812 \mathrm{e}+02$ \\
\hline 1.0017 & $1.897 \mathrm{e}+11$ & $2.017 \mathrm{e}+05$ & $5.330 \mathrm{e}+05$ & $3.717 e+02$ & $9.823 e+02$ \\
\hline 1.0441 & $1.519 \mathrm{e}+09$ & $1.734 \mathrm{e}+03$ & $4.509 e+03$ & $3.173 e+00$ & $8.248 e+00$ \\
\hline 1.1185 & $6.927 e+08$ & $8.906 e+02$ & $2.253 e+03$ & $1.608 e+00$ & $4.067 e+00$ \\
\hline 1.1421 & $4.262 \mathrm{e}+09$ & $5.680 e+03$ & $1.425 e+04$ & $1.021 \mathrm{e}+01$ & $2.562 \mathrm{e}+01$ \\
\hline 1.1732 & $1.532 \mathrm{e}+10$ & $2.139 e+04$ & $5.312 \mathrm{e}+04$ & $3.822 \mathrm{e}+01$ & $9.492 \mathrm{e}+01$ \\
\hline 1.2456 & $6.927 e+09$ & $1.071 \mathrm{e}+04$ & $2.601 \mathrm{e}+04$ & $1.889 \mathrm{e}+01$ & $4.586 \mathrm{e}+01$ \\
\hline 1.2745 & $2.389 \mathrm{e}+11$ & $3.843 e+05$ & $9.249 e+05$ & $6.739 e+02$ & $1.622 \mathrm{e}+03$ \\
\hline 1.3325 & $1.532 \mathrm{e}+10$ & $2.661 e+04$ & $6.299 e+04$ & $4.616 e+01$ & $1.093 e+02$ \\
\hline 1.3652 & $1.712 \mathrm{e}+09$ & $3.098 \mathrm{e}+03$ & $7.272 e+03$ & $5.343 e+00$ & $1.254 \mathrm{e}+01$ \\
\hline 1.4944 & $4.371 \mathrm{e}+09$ & $9.227 e+03$ & $2.098 e+04$ & $1.554 \mathrm{e}+01$ & $3.534 \mathrm{e}+01$ \\
\hline 1.5952 & $1.935 e+10$ & $4.561 \mathrm{e}+04$ & $1.015 e+05$ & $7.545 e+01$ & $1.679 \mathrm{e}+02$ \\
\hline Totals & $1.469 \mathrm{e}+14$ & $6.947 e+07$ & $2.176 e+08$ & $1.346 e+05$ & $4.215 e+05$ \\
\hline \multicolumn{6}{|c|}{ Results - Dose Point \# 2 - $(31.115,243.84,0) \mathrm{cm}$} \\
\hline Energy (MeV) & Activity (Photons/sec) & $\begin{array}{c}\text { Fluence Rate } \\
\mathrm{MeV} / \mathrm{cm}^{2} / \mathrm{sec} \\
\text { No Buildup }\end{array}$ & $\begin{array}{l}\text { Fluence Rate } \\
\mathrm{MeV} / \mathrm{cm}^{2} / \mathrm{sec} \\
\text { With Buildup }\end{array}$ & $\begin{array}{c}\text { Exposure Rate } \\
\mathrm{mR} / \mathrm{hr} \\
\text { No Buildup }\end{array}$ & $\begin{array}{c}\text { Exposure Rate } \\
\mathrm{mR} / \mathrm{hr} \\
\text { With Buildup }\end{array}$ \\
\hline 0.0419 & $1.492 \mathrm{e}+13$ & $1.207 \mathrm{e}-03$ & $4.374 \mathrm{e}-03$ & $4.749 \mathrm{e}-06$ & $1.721 \mathrm{e}-05$ \\
\hline 0.5874 & $1.139 \mathrm{e}+11$ & $4.141 \mathrm{e}+04$ & $1.387 e+05$ & $8.092 e+01$ & $2.710 \mathrm{e}+02$ \\
\hline 0.6617 & $1.312 \mathrm{e}+14$ & $5.852 \mathrm{e}+07$ & $1.858 \mathrm{e}+08$ & $1.134 \mathrm{e}+05$ & $3.603 e+05$ \\
\hline 0.7873 & $9.054 \mathrm{e}+10$ & $5.443 e+04$ & $1.605 \mathrm{e}+05$ & $1.037 \mathrm{e}+02$ & $3.058 \mathrm{e}+02$ \\
\hline 0.8759 & $8.603 e+10$ & $6.215 e+04$ & $1.754 \mathrm{e}+05$ & $1.169 \mathrm{e}+02$ & $3.298 \mathrm{e}+02$ \\
\hline 1.0017 & $1.897 \mathrm{e}+11$ & $1.727 e+05$ & $4.616 \mathrm{e}+05$ & $3.183 e+02$ & $8.506 \mathrm{e}+02$ \\
\hline 1.0441 & $1.519 \mathrm{e}+09$ & $1.486 e+03$ & $3.906 \mathrm{e}+03$ & $2.719 \mathrm{e}+00$ & $7.144 \mathrm{e}+00$ \\
\hline 1.1185 & $6.927 e+08$ & $7.636 e+02$ & $1.953 e+03$ & $1.378 \mathrm{e}+00$ & $3.525 e+00$ \\
\hline 1.1421 & $4.262 \mathrm{e}+09$ & $4.871 \mathrm{e}+03$ & $1.235 e+04$ & $8.755 e+00$ & $2.220 \mathrm{e}+01$ \\
\hline 1.1732 & $1.532 \mathrm{e}+10$ & $1.835 e+04$ & $4.604 e+04$ & $3.279 e+01$ & $8.228 \mathrm{e}+01$ \\
\hline 1.2456 & $6.927 \mathrm{e}+09$ & $9.198 \mathrm{e}+03$ & $2.256 \mathrm{e}+04$ & $1.622 \mathrm{e}+01$ & $3.977 \mathrm{e}+01$ \\
\hline 1.2745 & $2.389 \mathrm{e}+11$ & $3.300 \mathrm{e}+05$ & $8.022 e+05$ & $5.787 e+02$ & $1.407 \mathrm{e}+03$ \\
\hline 1.3325 & $1.532 \mathrm{e}+10$ & $2.286 \mathrm{e}+04$ & $5.465 e+04$ & $3.965 e+01$ & $9.482 \mathrm{e}+01$ \\
\hline 1.3652 & $1.712 \mathrm{e}+09$ & $2.662 \mathrm{e}+03$ & $6.310 \mathrm{e}+03$ & $4.591 \mathrm{e}+00$ & $1.088 \mathrm{e}+01$ \\
\hline 1.4944 & $4.371 \mathrm{e}+09$ & $7.936 \mathrm{e}+03$ & $1.822 \mathrm{e}+04$ & $1.337 \mathrm{e}+01$ & $3.068 \mathrm{e}+01$ \\
\hline 1.5952 & $1.935 \mathrm{e}+10$ & $3.926 \mathrm{e}+04$ & $8.818 \mathrm{e}+04$ & $6.494 \mathrm{e}+01$ & $1.459 \mathrm{e}+02$ \\
\hline Totals & $1.469 \mathrm{e}+14$ & $5.928 \mathrm{e}+07$ & $1.878 \mathrm{e}+08$ & $1.148 \mathrm{e}+05$ & $3.639 \mathrm{e}+05$ \\
\hline \multicolumn{6}{|c|}{ Results - Dose Point \# 3 - $(56.515,243.84,0) \mathrm{cm}$} \\
\hline Energy (MeV) & Activity (Photons/sec) & $\begin{array}{c}\text { Fluence Rate } \\
\mathrm{MeV} / \mathrm{cm}^{2} / \mathrm{sec} \\
\text { No Buildup }\end{array}$ & $\begin{array}{l}\text { Fluence Rate } \\
\mathrm{MeV} / \mathrm{cm}^{2} / \mathrm{sec} \\
\text { With Buildup }\end{array}$ & $\begin{array}{c}\text { Exposure Rate } \\
\mathrm{mR} / \mathrm{hr} \\
\text { No Buildup }\end{array}$ & $\begin{array}{c}\text { Exposure Rate } \\
\mathrm{mR} / \mathrm{hr} \\
\text { With Buildup }\end{array}$ \\
\hline 0.0419 & $1.492 \mathrm{e}+13$ & $1.285 \mathrm{e}-03$ & $4.504 \mathrm{e}-03$ & $5.056 \mathrm{e}-06$ & $1.772 \mathrm{e}-05$ \\
\hline 0.5874 & $1.139 \mathrm{e}+11$ & $2.024 e+04$ & $6.844 e+04$ & $3.956 \mathrm{e}+01$ & $1.337 e+02$ \\
\hline 0.6617 & $1.312 \mathrm{e}+14$ & $2.864 \mathrm{e}+07$ & $9.170 \mathrm{e}+07$ & $5.552 e+04$ & $1.778 \mathrm{e}+05$ \\
\hline 0.7873 & $9.054 \mathrm{e}+10$ & $2.667 e+04$ & $7.918 e+04$ & $5.083 e+01$ & $1.509 \mathrm{e}+02$ \\
\hline 0.8759 & $8.603 e+10$ & $3.048 \mathrm{e}+04$ & $8.651 e+04$ & $5.732 e+01$ & $1.627 e+02$ \\
\hline 1.0017 & $1.897 \mathrm{e}+11$ & $8.479 e+04$ & $2.277 e+05$ & $1.563 e+02$ & $4.196 \mathrm{e}+02$ \\
\hline 1.0441 & $1.519 e+09$ & $7.297 e+02$ & $1.926 \mathrm{e}+03$ & $1.335 \mathrm{e}+00$ & $3.524 \mathrm{e}+00$ \\
\hline 1.1185 & $6.927 e+08$ & $3.751 e+02$ & $9.631 e+02$ & $6.770 \mathrm{e}-01$ & $1.739 \mathrm{e}+00$ \\
\hline 1.1421 & $4.262 e+09$ & $2.393 e+03$ & $6.094 e+03$ & $4.301 \mathrm{e}+00$ & $1.095 e+01$ \\
\hline 1.1732 & $1.532 \mathrm{e}+10$ & $9.014 e+03$ & $2.271 e+04$ & $1.611 \mathrm{e}+01$ & $4.059 e+01$ \\
\hline 1.2456 & $6.927 e+09$ & $4.520 \mathrm{e}+03$ & $1.113 e+04$ & $7.970 e+00$ & $1.962 \mathrm{e}+01$ \\
\hline 1.2745 & $2.389 \mathrm{e}+11$ & $1.622 \mathrm{e}+05$ & $3.957 e+05$ & $2.844 e+02$ & $6.939 e+02$ \\
\hline 1.3325 & $1.532 \mathrm{e}+10$ & $1.124 \mathrm{e}+04$ & $2.696 \mathrm{e}+04$ & $1.949 \mathrm{e}+01$ & $4.677 \mathrm{e}+01$ \\
\hline 1.3652 & $1.712 \mathrm{e}+09$ & $1.309 e+03$ & $3.113 e+03$ & $2.257 e+00$ & $5.367 e+00$ \\
\hline 1.4944 & $4.371 \mathrm{e}+09$ & $3.903 e+03$ & $8.986 e+03$ & $6.574 e+00$ & $1.513 e+01$ \\
\hline
\end{tabular}




\begin{tabular}{|l|l|l|l|l|l|}
\hline 1.5952 & $1.935 \mathrm{e}+10$ & $1.931 \mathrm{e}+04$ & $4.350 \mathrm{e}+04$ & $3.195 \mathrm{e}+01$ & $7.195 \mathrm{e}+01$ \\
\hline Totals & $1.469 \mathrm{e}+14$ & $2.901 \mathrm{e}+07$ & $9.268 \mathrm{e}+07$ & $5.620 \mathrm{e}+04$ & $1.795 \mathrm{e}+05$ \\
\hline
\end{tabular}


MicroShield 7.02

DOE (7.02-0000)

\begin{tabular}{|c|c|c|c|}
\hline Date & \multicolumn{2}{c|}{ Checked } \\
\hline 3/16/Z009 & Run Date & Run Time & Duration \\
\hline Filename & March 5, 2009 & $2: 26: 13$ PM & $00: 00: 02$ \\
\hline KBC40179_5.ms7 & Project Info \\
\hline Case Title & \multicolumn{2}{|c|}{ Sample Bottle Shield } \\
\hline Description & Sample Bottle Settler 400ml in cart shield (1.4in Pb) \\
\hline Geometry & 7-Cylinder Volume - Side Shields \\
\hline
\end{tabular}

\begin{tabular}{|c|c|c|c|c|}
\hline \multicolumn{5}{|c|}{ Source Dimensions } \\
\hline & Height & \multicolumn{3}{|c|}{$2.195 \mathrm{~cm}$ (0.9 in) } \\
\hline & Radius & \multicolumn{3}{|c|}{$7.62 \mathrm{~cm}(3.0 \mathrm{in})$} \\
\hline \multicolumn{5}{|c|}{ Dose Points } \\
\hline A & \multicolumn{2}{|c|}{$\mathrm{x}$} & $\mathrm{Y}$ & $z$ \\
\hline$\# 1$ & \multicolumn{2}{|c|}{$14.194 \mathrm{~cm}$ (5.6 in) } & $1.1 \mathrm{~cm}(0.4 \mathrm{in})$ & $0.0 \mathrm{~cm}(0.0 \mathrm{in})$ \\
\hline \#2 & \multicolumn{2}{|c|}{$42.164 \mathrm{~cm}(1 \mathrm{ft} 4.6 \mathrm{in})$} & $1.1 \mathrm{~cm}(0.4 \mathrm{in})$ & $0.0 \mathrm{~cm}(0.0 \mathrm{in})$ \\
\hline$\# 3$ & \multicolumn{2}{|c|}{$111.654 \mathrm{~cm}$ (3 ft $8.0 \mathrm{in})$} & $1.1 \mathrm{~cm}(0.4 \mathrm{in})$ & $0.0 \mathrm{~cm}(0.0 \mathrm{in})$ \\
\hline \#4 & \multicolumn{2}{|c|}{$316.382 \mathrm{~cm}$ (10 ft $4.6 \mathrm{in})$} & $1.1 \mathrm{~cm}(0.4 \mathrm{in})$ & $0.0 \mathrm{~cm}(0.0 \mathrm{in})$ \\
\hline \multicolumn{5}{|c|}{ Shields } \\
\hline & Shield $\mathrm{N}$ & Dimension & Material & Density \\
\hline & Source & $400.4 \mathrm{~cm}^{3}$ & Concrete & 3 \\
\hline & Shield 1 & $3.556 \mathrm{~cm}$ & Lead & 11.34 \\
\hline & Transition & & Air & 0.00122 \\
\hline & Air Gap & & Air & 0.00122 \\
\hline & Wall Clad & $.478 \mathrm{~cm}$ & Water & 1 \\
\hline & Top Clad & $.478 \mathrm{~cm}$ & Water & 1 \\
\hline
\end{tabular}

Source Input: Grouping Method - Standard Indices

Number of Groups: 25

Lower Energy Cutoff: 0.015

Photons < 0.015: Excluded

Library: Grove

\begin{tabular}{|c|c|c|c|c|}
\hline Nuclide & $\mathrm{Ci}$ & $\mathrm{Bq}$ & $\mu \mathrm{Ci} / \mathrm{cm}^{3}$ & $\mathrm{~Bq} / \mathrm{cm}^{3}$ \\
\hline Am-241 & $1.9139 \mathrm{e}-001$ & $7.0815 \mathrm{e}+009$ & $4.7800 \mathrm{e}+002$ & $1.7686 \mathrm{e}+007$ \\
\hline Ba-137m & $2.9583 \mathrm{e}+000$ & $1.0946 \mathrm{e}+011$ & $7.3883 \mathrm{e}+003$ & $2.7337 \mathrm{e}+008$ \\
\hline Co-60 & $3.1071 \mathrm{e}-004$ & $1.1496 \mathrm{e}+007$ & $7.7600 \mathrm{e}-001$ & $2.8712 \mathrm{e}+004$ \\
\hline Cs-134 & $1.1411 \mathrm{e}-003$ & $4.2222 \mathrm{e}+007$ & $2.8500 \mathrm{e}+000$ & $1.0545 \mathrm{e}+005$ \\
\hline Cs-137 & $3.1271 \mathrm{e}+000$ & $1.1570 \mathrm{e}+011$ & $7.8100 \mathrm{e}+003$ & $2.8897 \mathrm{e}+008$ \\
\hline Eu-154 & $1.3493 \mathrm{e}-002$ & $4.9926 \mathrm{e}+008$ & $3.3700 \mathrm{e}+001$ & $1.2469 \mathrm{e}+006$ \\
\hline Eu-155 & $4.7247 \mathrm{e}-003$ & $1.7481 \mathrm{e}+008$ & $1.1800 \mathrm{e}+001$ & $4.3660 \mathrm{e}+005$ \\
\hline Np-237 & $1.1451 \mathrm{e}-005$ & $4.2370 \mathrm{e}+005$ & $2.8600 \mathrm{e}-002$ & $1.0582 \mathrm{e}+003$ \\
\hline Pu-238 & $2.7227 \mathrm{e}-002$ & $1.0074 \mathrm{e}+009$ & $6.8000 \mathrm{e}+001$ & $2.5160 \mathrm{e}+006$ \\
\hline Pu-239 & $1.1491 \mathrm{e}-001$ & $4.2519 \mathrm{e}+009$ & $2.8700 \mathrm{e}+002$ & $1.0619 \mathrm{e}+007$ \\
\hline Pu-240 & $6.9269 \mathrm{e}-002$ & $2.5630 \mathrm{e}+009$ & $1.7300 \mathrm{e}+002$ & $6.4010 \mathrm{e}+006$ \\
\hline Pu-241 & $2.3704 \mathrm{e}+000$ & $8.7704 \mathrm{e}+010$ & $5.9200 \mathrm{e}+003$ & $2.1904 \mathrm{e}+008$ \\
\hline Pu-242 & $2.5065 \mathrm{e}-005$ & $9.2741 \mathrm{e}+005$ & $6.2600 \mathrm{e}-002$ & $2.3162 \mathrm{e}+003$ \\
\hline Sm-151 & $1.1411 \mathrm{e}-003$ & $4.2222 \mathrm{e}+007$ & $2.8500 \mathrm{e}+000$ & $1.0545 \mathrm{e}+005$ \\
\hline Sr-90 & $3.4995 \mathrm{e}+000$ & $1.2948 \mathrm{e}+011$ & $8.7400 \mathrm{e}+003$ & $3.2338 \mathrm{e}+008$ \\
\hline Tc-99 & $8.4484 \mathrm{e}-004$ & $3.1259 \mathrm{e}+007$ & $2.1100 \mathrm{e}+000$ & $7.8070 \mathrm{e}+004$ \\
\hline Y-90 & $3.4995 \mathrm{e}+000$ & $1.2948 \mathrm{e}+011$ & $8.7400 \mathrm{e}+003$ & $3.2338 \mathrm{e}+008$ \\
\hline
\end{tabular}




\begin{tabular}{|c|c|c|c|c|c|}
\hline \multicolumn{6}{|c|}{ Results - Dose Point \# $1-(14.194,1.1,0) \mathrm{cm}$} \\
\hline Energy (MeV) & Activity (Photons/sec) & $\begin{array}{l}\text { Fluence Rate } \\
\mathrm{MeV} / \mathrm{cm}^{2} / \mathrm{sec} \\
\text { No Buildup }\end{array}$ & $\begin{array}{l}\text { Fluence Rate } \\
\mathrm{MeV} / \mathrm{cm}^{2} / \mathrm{sec} \\
\text { With Buildup }\end{array}$ & $\begin{array}{c}\text { Exposure Rate } \\
\mathrm{mR} / \mathrm{hr} \\
\text { No Buildup }\end{array}$ & $\begin{array}{c}\text { Exposure Rate } \\
\mathrm{mR} / \mathrm{hr} \\
\text { With Buildup }\end{array}$ \\
\hline 0.02 & $1.248 \mathrm{e}+04$ & $0.000 \mathrm{e}+00$ & $1.015 \mathrm{e}-27$ & $0.000 \mathrm{e}+00$ & $3.517 \mathrm{e}-29$ \\
\hline 0.03 & $6.625 e+09$ & $0.000 e+00$ & $8.309 \mathrm{e}-22$ & $0.000 \mathrm{e}+00$ & $8.234 \mathrm{e}-24$ \\
\hline 0.04 & $1.655 \mathrm{e}+09$ & $3.462 \mathrm{e}-235$ & $2.845 \mathrm{e}-22$ & $1.531 \mathrm{e}-237$ & $1.258 \mathrm{e}-24$ \\
\hline 0.05 & $3.746 \mathrm{e}+07$ & $3.858 \mathrm{e}-130$ & $8.302 \mathrm{e}-24$ & $1.028 \mathrm{e}-132$ & $2.212 \mathrm{e}-26$ \\
\hline 0.06 & $2.558 \mathrm{e}+09$ & $8.768 \mathrm{e}-78$ & $7.126 \mathrm{e}-22$ & $1.741 \mathrm{e}-80$ & $1.415 \mathrm{e}-24$ \\
\hline 0.08 & $5.434 \mathrm{e}+07$ & $2.071 e-36$ & $2.263 e-23$ & $3.277 e-39$ & $3.580 \mathrm{e}-26$ \\
\hline 0.1 & $2.402 \mathrm{e}+08$ & $2.585 e-92$ & $3.967 e-06$ & $3.955 e-95$ & $6.070 \mathrm{e}-09$ \\
\hline 0.15 & $7.255 \mathrm{e}+03$ & $4.346 \mathrm{e}-36$ & $1.379 \mathrm{e}-25$ & $7.157 e-39$ & $2.271 \mathrm{e}-28$ \\
\hline 0.2 & $3.410 \mathrm{e}+07$ & $6.217 \mathrm{e}-15$ & $9.339 \mathrm{e}-15$ & $1.097 \mathrm{e}-17$ & $1.648 \mathrm{e}-17$ \\
\hline 0.3 & $1.495 \mathrm{e}+04$ & $6.109 \mathrm{e}-08$ & $9.431 \mathrm{e}-08$ & $1.159 \mathrm{e}-10$ & $1.789 \mathrm{e}-10$ \\
\hline 0.4 & $3.562 \mathrm{e}+06$ & $1.719 \mathrm{e}-02$ & $3.011 \mathrm{e}-02$ & $3.350 \mathrm{e}-05$ & $5.867 e-05$ \\
\hline 0.5 & $1.697 e+06$ & $1.796 \mathrm{e}-01$ & $3.405 \mathrm{e}-01$ & $3.526 \mathrm{e}-04$ & $6.683 e-04$ \\
\hline 0.6 & $9.858 \mathrm{e}+10$ & $5.611 e+04$ & $1.106 \mathrm{e}+05$ & $1.095 \mathrm{e}+02$ & $2.159 \mathrm{e}+02$ \\
\hline 0.8 & $2.344 e+08$ & $8.187 e+02$ & $1.701 \mathrm{e}+03$ & $1.557 e+00$ & $3.235 e+00$ \\
\hline 1.0 & $1.663 e+08$ & $1.589 e+03$ & $3.355 e+03$ & $2.929 \mathrm{e}+00$ & $6.184 e+00$ \\
\hline 1.5 & $2.076 \mathrm{e}+08$ & $7.274 e+03$ & $1.464 \mathrm{e}+04$ & $1.224 \mathrm{e}+01$ & $2.463 e+01$ \\
\hline Totals & $1.104 \mathrm{e}+11$ & $6.579 \mathrm{e}+04$ & $1.303 e+05$ & $1.263 e+02$ & $2.499 \mathrm{e}+02$ \\
\hline \multicolumn{6}{|c|}{ Results - Dose Point \# 2 - $(42.164,1.1,0) \mathrm{cm}$} \\
\hline Energy (MeV) & Activity (Photons/sec) & $\begin{array}{c}\text { Fluence Rate } \\
\mathrm{MeV} / \mathrm{cm}^{2} / \mathrm{sec} \\
\text { No Buildup }\end{array}$ & $\begin{array}{l}\text { Fluence Rate } \\
\mathrm{MeV} / \mathrm{cm}^{2} / \mathrm{sec} \\
\text { With Buildup }\end{array}$ & $\begin{array}{c}\text { Exposure Rate } \\
\mathrm{mR} / \mathrm{hr} \\
\text { No Buildup }\end{array}$ & $\begin{array}{c}\text { Exposure Rate } \\
\mathrm{mR} / \mathrm{hr} \\
\text { With Buildup }\end{array}$ \\
\hline 0.02 & $1.248 \mathrm{e}+04$ & $0.000 \mathrm{e}+00$ & $9.948 \mathrm{e}-29$ & $0.000 \mathrm{e}+00$ & $3.446 \mathrm{e}-30$ \\
\hline 0.03 & $6.625 e+09$ & $0.000 \mathrm{e}+00$ & $8.140 \mathrm{e}-23$ & $0.000 e+00$ & $8.068 \mathrm{e}-25$ \\
\hline 0.04 & $1.655 e+09$ & $5.231 \mathrm{e}-236$ & $2.787 e-23$ & $2.314 \mathrm{e}-238$ & $1.233 \mathrm{e}-25$ \\
\hline 0.05 & $3.746 e+07$ & $5.266 \mathrm{e}-131$ & $8.134 \mathrm{e}-25$ & $1.403 e-133$ & $2.167 \mathrm{e}-27$ \\
\hline 0.06 & $2.558 \mathrm{e}+09$ & $1.039 \mathrm{e}-78$ & $6.982 \mathrm{e}-23$ & $2.063 e-81$ & $1.387 \mathrm{e}-25$ \\
\hline 0.08 & $5.434 \mathrm{e}+07$ & $1.963 e-37$ & $2.217 e-24$ & $3.107 e-40$ & $3.508 \mathrm{e}-27$ \\
\hline 0.1 & $2.402 \mathrm{e}+08$ & $3.236 \mathrm{e}-93$ & $3.887 e-07$ & $4.951 \mathrm{e}-96$ & $5.947 \mathrm{e}-10$ \\
\hline 0.15 & $7.255 e+03$ & $4.137 e-37$ & $1.351 \mathrm{e}-26$ & $6.812 e-40$ & $2.225 \mathrm{e}-29$ \\
\hline 0.2 & $3.410 \mathrm{e}+07$ & $5.442 \mathrm{e}-16$ & $8.176 \mathrm{e}-16$ & $9.605 e-19$ & $1.443 \mathrm{e}-18$ \\
\hline 0.3 & $1.495 e+04$ & $5.138 \mathrm{e}-09$ & $7.938 \mathrm{e}-09$ & $9.747 e-12$ & $1.506 \mathrm{e}-11$ \\
\hline 0.4 & $3.562 \mathrm{e}+06$ & $1.443 e-03$ & $2.533 e-03$ & $2.811 \mathrm{e}-06$ & $4.935 e-06$ \\
\hline 0.5 & $1.697 \mathrm{e}+06$ & $1.514 \mathrm{e}-02$ & $2.883 e-02$ & $2.973 \mathrm{e}-05$ & $5.659 \mathrm{e}-05$ \\
\hline 0.6 & $9.858 \mathrm{e}+10$ & $4.760 \mathrm{e}+03$ & $9.439 \mathrm{e}+03$ & $9.290 \mathrm{e}+00$ & $1.842 \mathrm{e}+01$ \\
\hline 0.8 & $2.344 \mathrm{e}+08$ & $7.027 e+01$ & $1.473 e+02$ & $1.337 \mathrm{e}-01$ & $2.803 e-01$ \\
\hline 1.0 & $1.663 e+08$ & $1.378 \mathrm{e}+02$ & $2.942 \mathrm{e}+02$ & $2.539 e-01$ & $5.423 e-01$ \\
\hline 1.5 & $2.076 \mathrm{e}+08$ & $6.428 \mathrm{e}+02$ & $1.313 e+03$ & $1.081 \mathrm{e}+00$ & $2.209 \mathrm{e}+00$ \\
\hline Totals & $1.104 \mathrm{e}+11$ & $5.611 \mathrm{e}+03$ & $1.119 \mathrm{e}+04$ & $1.076 \mathrm{e}+01$ & $2.146 e+01$ \\
\hline \multicolumn{6}{|c|}{ Results - Dose Point \# $3-(111.654,1.1,0) \mathrm{cm}$} \\
\hline Energy (MeV) & Activity (Photons/sec) & $\begin{array}{c}\text { Fluence Rate } \\
\mathrm{MeV} / \mathrm{cm}^{2} / \mathrm{sec} \\
\text { No Buildup }\end{array}$ & $\begin{array}{l}\text { Fluence Rate } \\
\mathrm{MeV} / \mathrm{cm}^{2} / \mathrm{sec} \\
\text { With Buildup }\end{array}$ & $\begin{array}{c}\text { Exposure Rate } \\
\text { mR/hr } \\
\text { No Buildup }\end{array}$ & $\begin{array}{c}\text { Exposure Rate } \\
\mathrm{mR} / \mathrm{hr} \\
\text { With Buildup }\end{array}$ \\
\hline 0.02 & $1.248 \mathrm{e}+04$ & $0.000 \mathrm{e}+00$ & $1.399 \mathrm{e}-29$ & $0.000 \mathrm{e}+00$ & $4.845 \mathrm{e}-31$ \\
\hline 0.03 & $6.625 e+09$ & $0.000 \mathrm{e}+00$ & $1.145 \mathrm{e}-23$ & $0.000 \mathrm{e}+00$ & $1.134 \mathrm{e}-25$ \\
\hline 0.04 & $1.655 e+09$ & $7.028 \mathrm{e}-237$ & $3.920 \mathrm{e}-24$ & $3.108 \mathrm{e}-239$ & $1.734 \mathrm{e}-26$ \\
\hline 0.05 & $3.746 \mathrm{e}+07$ & $6.957 \mathrm{e}-132$ & $1.144 \mathrm{e}-25$ & $1.853 e-134$ & $3.047 \mathrm{e}-28$ \\
\hline 0.06 & $2.558 \mathrm{e}+09$ & $1.345 \mathrm{e}-79$ & $9.818 \mathrm{e}-24$ & $2.671 \mathrm{e}-82$ & $1.950 \mathrm{e}-26$ \\
\hline 0.08 & $5.434 \mathrm{e}+07$ & $2.533 e-38$ & $3.117 \mathrm{e}-25$ & $4.009 e-41$ & $4.933 e-28$ \\
\hline 0.1 & $2.402 \mathrm{e}+08$ & $4.270 \mathrm{e}-94$ & $5.466 \mathrm{e}-08$ & $6.532 \mathrm{e}-97$ & $8.362 \mathrm{e}-11$ \\
\hline 0.15 & $7.255 e+03$ & $5.402 \mathrm{e}-38$ & $1.900 \mathrm{e}-27$ & $8.895 e-41$ & $3.129 \mathrm{e}-30$ \\
\hline 0.2 & $3.410 \mathrm{e}+07$ & $7.109 \mathrm{e}-17$ & $1.068 \mathrm{e}-16$ & $1.255 \mathrm{e}-19$ & $1.885 \mathrm{e}-19$ \\
\hline 0.3 & $1.495 e+04$ & $6.750 \mathrm{e}-10$ & $1.043 e-09$ & $1.281 \mathrm{e}-12$ & $1.979 \mathrm{e}-12$ \\
\hline 0.4 & $3.562 e+06$ & $1.906 \mathrm{e}-04$ & $3.350 \mathrm{e}-04$ & $3.714 \mathrm{e}-07$ & $6.527 \mathrm{e}-07$ \\
\hline
\end{tabular}


KBC-40179 rev 1

\begin{tabular}{|c|c|c|c|c|c|}
\hline 0.5 & $1.697 \mathrm{e}+06$ & $2.011 \mathrm{e}-03$ & $3.834 \mathrm{e}-03$ & $3.947 \mathrm{e}-06$ & $7.526 \mathrm{e}-06$ \\
\hline 0.6 & $9.858 \mathrm{e}+10$ & $6.344 \mathrm{e}+02$ & $1.261 \mathrm{e}+03$ & $1.238 \mathrm{e}+00$ & $2.462 \mathrm{e}+00$ \\
\hline 0.8 & $2.344 e+08$ & $9.421 e+00$ & $1.982 e+01$ & $1.792 \mathrm{e}-02$ & $3.771 \mathrm{e}-02$ \\
\hline 1.0 & $1.663 e+08$ & $1.855 e+01$ & $3.979 e+01$ & $3.420 \mathrm{e}-02$ & $7.334 \mathrm{e}-02$ \\
\hline 1.5 & $2.076 e+08$ & $8.719 e+01$ & $1.791 \mathrm{e}+02$ & $1.467 e-01$ & $3.013 e-01$ \\
\hline Totals & $1.104 \mathrm{e}+11$ & $7.496 \mathrm{e}+02$ & $1.500 \mathrm{e}+03$ & $1.437 e+00$ & $2.874 \mathrm{e}+00$ \\
\hline \multicolumn{6}{|c|}{ Results - Dose Point \# 4 - $(316.382,1.1,0) \mathrm{cm}$} \\
\hline Energy (MeV) & Activity (Photons/sec) & $\begin{array}{l}\text { Fluence Rate } \\
\mathrm{MeV} / \mathrm{cm}^{2} / \mathrm{sec} \\
\text { No Buildup }\end{array}$ & $\begin{array}{l}\text { Fluence Rate } \\
\mathrm{MeV} / \mathrm{cm}^{2} / \mathrm{sec} \\
\text { With Buildup }\end{array}$ & $\begin{array}{c}\text { Exposure Rate } \\
\mathrm{mR} / \mathrm{hr} \\
\text { No Buildup }\end{array}$ & $\begin{array}{c}\text { Exposure Rate } \\
\mathrm{mR} / \mathrm{hr} \\
\text { With Buildup }\end{array}$ \\
\hline 0.02 & $1.248 \mathrm{e}+04$ & $0.000 e+00$ & $1.739 \mathrm{e}-30$ & $0.000 \mathrm{e}+00$ & $6.023 e-32$ \\
\hline 0.03 & $6.625 e+09$ & $0.000 \mathrm{e}+00$ & $1.423 e-24$ & $0.000 \mathrm{e}+00$ & $1.410 \mathrm{e}-26$ \\
\hline 0.04 & $1.655 e+09$ & $8.020 \mathrm{e}-238$ & $4.872 \mathrm{e}-25$ & $3.547 \mathrm{e}-240$ & $2.155 \mathrm{e}-27$ \\
\hline 0.05 & $3.746 e+07$ & $7.978 \mathrm{e}-133$ & $1.422 \mathrm{e}-26$ & $2.125 \mathrm{e}-135$ & $3.787 e-29$ \\
\hline 0.06 & $2.558 \mathrm{e}+09$ & $1.545 e-80$ & $1.220 \mathrm{e}-24$ & $3.068 \mathrm{e}-83$ & $2.424 \mathrm{e}-27$ \\
\hline 0.08 & $5.434 \mathrm{e}+07$ & $2.929 \mathrm{e}-39$ & $3.874 \mathrm{e}-26$ & $4.635 e-42$ & $6.131 \mathrm{e}-29$ \\
\hline 0.1 & $2.402 e+08$ & $4.964 \mathrm{e}-95$ & $6.794 \mathrm{e}-09$ & $7.594 \mathrm{e}-98$ & $1.039 \mathrm{e}-11$ \\
\hline 0.15 & $7.255 e+03$ & $6.312 \mathrm{e}-39$ & $2.362 \mathrm{e}-28$ & $1.039 \mathrm{e}-41$ & $3.889 \mathrm{e}-31$ \\
\hline 0.2 & $3.410 \mathrm{e}+07$ & $8.341 \mathrm{e}-18$ & $1.253 e-17$ & $1.472 \mathrm{e}-20$ & $2.212 \mathrm{e}-20$ \\
\hline 0.3 & $1.495 e+04$ & $7.974 \mathrm{e}-11$ & $1.233 \mathrm{e}-10$ & $1.513 \mathrm{e}-13$ & $2.338 \mathrm{e}-13$ \\
\hline 0.4 & $3.562 \mathrm{e}+06$ & $2.263 \mathrm{e}-05$ & $3.981 \mathrm{e}-05$ & $4.409 \mathrm{e}-08$ & 7.757e-08 \\
\hline 0.5 & $1.697 e+06$ & $2.396 \mathrm{e}-04$ & $4.577 \mathrm{e}-04$ & $4.704 \mathrm{e}-07$ & $8.983 \mathrm{e}-07$ \\
\hline 0.6 & $9.858 \mathrm{e}+10$ & $7.585 e+01$ & $1.511 \mathrm{e}+02$ & $1.480 \mathrm{e}-01$ & $2.949 \mathrm{e}-01$ \\
\hline 0.8 & $2.344 \mathrm{e}+08$ & $1.132 e+00$ & $2.387 e+00$ & $2.152 e-03$ & $4.541 \mathrm{e}-03$ \\
\hline 1.0 & $1.663 e+08$ & $2.236 \mathrm{e}+00$ & $4.810 \mathrm{e}+00$ & $4.121 \mathrm{e}-03$ & $8.867 \mathrm{e}-03$ \\
\hline 1.5 & $2.076 e+08$ & $1.057 \mathrm{e}+01$ & $2.179 \mathrm{e}+01$ & $1.778 \mathrm{e}-02$ & $3.666 \mathrm{e}-02$ \\
\hline Totals & $1.104 \mathrm{e}+11$ & $8.978 \mathrm{e}+01$ & $1.801 \mathrm{e}+02$ & $1.721 \mathrm{e}-01$ & $3.450 \mathrm{e}-01$ \\
\hline
\end{tabular}


MicroShield 7.02

DOE (7.02-0000)

\begin{tabular}{|c|c|c|c|}
\hline \multicolumn{1}{|c|}{ Date } & Run Time & Duration \\
\hline $3 / 16 / 2009$ & Run Date & 2:33:06 PM \\
\hline Filename & March 5, 2009 & $00: 00: 03$ \\
\hline KBC40179_6.ms7 & Project Info \\
\hline Case Title & Settler Sample Unsh \\
\hline Description & Sample Bottle Settler 400ml at Distances up to 10ft \\
\hline Geometry & 7- Cylinder Volume - Side Shields \\
\hline
\end{tabular}

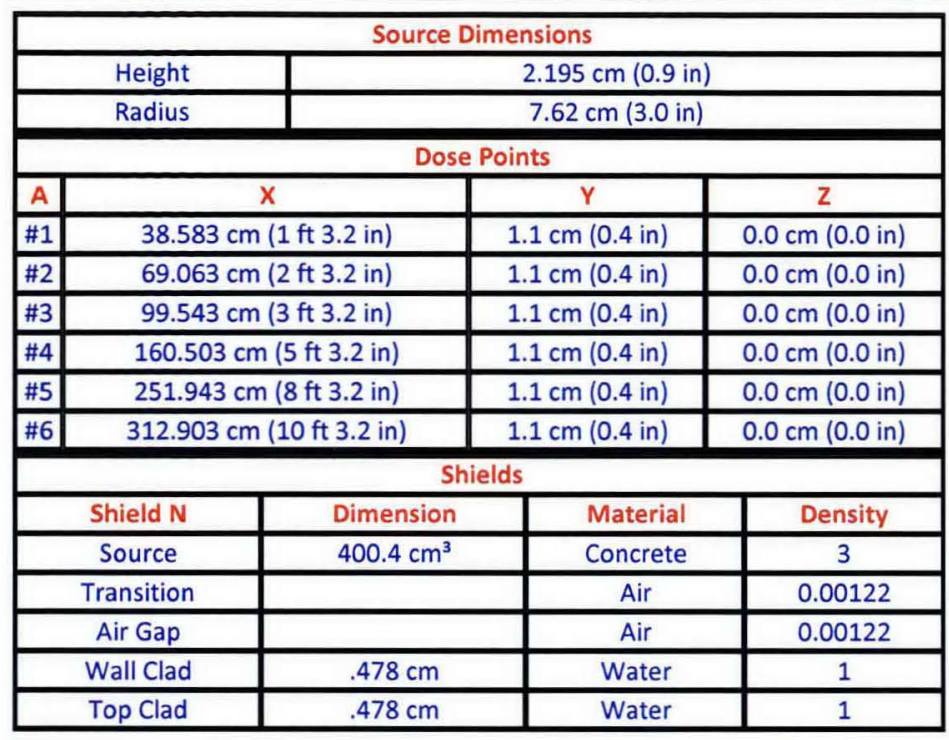

\begin{tabular}{|c|c|c|c|c|}
\hline \multicolumn{5}{|c|}{$\begin{array}{c}\text { Source Input: Grouping Method - Linear Energy } \\
\text { Number of Groups: } 25 \\
\text { Lower Energy Cutoff: } 0.015 \\
\text { Photons < } 0.015 \text { : Excluded } \\
\text { Library: Grove }\end{array}$} \\
\hline Nuclide & $\mathrm{Ci}$ & $\mathrm{Bq}$ & $\mu \mathrm{Ci} / \mathrm{cm}^{3}$ & $\mathrm{~Bq} / \mathrm{cm}^{3}$ \\
\hline$A m-241$ & $1.9139 \mathrm{e}-001$ & $7.0815 e+009$ & $4.7800 e+002$ & $1.7686 \mathrm{e}+007$ \\
\hline Ba-137m & $2.9583 e+000$ & $1.0946 \mathrm{e}+011$ & $7.3883 e+003$ & $2.7337 e+008$ \\
\hline Co-60 & $3.1071 \mathrm{e}-004$ & $1.1496 \mathrm{e}+007$ & $7.7600 \mathrm{e}-001$ & $2.8712 e+004$ \\
\hline Cs-134 & $1.1411 \mathrm{e}-003$ & $4.2222 \mathrm{e}+007$ & $2.8500 e+000$ & $1.0545 e+005$ \\
\hline Cs-137 & $3.1271 \mathrm{e}+000$ & $1.1570 \mathrm{e}+011$ & $7.8100 e+003$ & $2.8897 e+008$ \\
\hline Eu-154 & $1.3493 e-002$ & $4.9926 e+008$ & $3.3700 e+001$ & $1.2469 e+006$ \\
\hline Eu-155 & $4.7247 \mathrm{e}-003$ & $1.7481 \mathrm{e}+008$ & $1.1800 \mathrm{e}+001$ & $4.3660 \mathrm{e}+005$ \\
\hline $\mathrm{Np}-237$ & $1.1451 \mathrm{e}-005$ & $4.2370 \mathrm{e}+005$ & $2.8600 \mathrm{e}-002$ & $1.0582 \mathrm{e}+003$ \\
\hline $\mathrm{Pu}-238$ & $2.7227 \mathrm{e}-002$ & $1.0074 \mathrm{e}+009$ & $6.8000 \mathrm{e}+001$ & $2.5160 \mathrm{e}+006$ \\
\hline Pu-239 & $1.1491 \mathrm{e}-001$ & $4.2519 \mathrm{e}+009$ & $2.8700 \mathrm{e}+002$ & $1.0619 e+007$ \\
\hline $\mathrm{Pu}-240$ & $6.9269 \mathrm{e}-002$ & $2.5630 \mathrm{e}+009$ & $1.7300 \mathrm{e}+002$ & $6.4010 e+006$ \\
\hline Pu-241 & $2.3704 \mathrm{e}+000$ & $8.7704 \mathrm{e}+010$ & $5.9200 e+003$ & $2.1904 \mathrm{e}+008$ \\
\hline Pu-242 & $2.5065 \mathrm{e}-005$ & $9.2741 \mathrm{e}+005$ & $6.2600 \mathrm{e}-002$ & $2.3162 e+003$ \\
\hline Sm-151 & $1.1411 \mathrm{e}-003$ & $4.2222 \mathrm{e}+007$ & $2.8500 e+000$ & $1.0545 e+005$ \\
\hline Sr-90 & $3.4995 \mathrm{e}+000$ & $1.2948 \mathrm{e}+011$ & $8.7400 e+003$ & $3.2338 e+008$ \\
\hline Tc-99 & $8.4484 \mathrm{e}-004$ & $3.1259 e+007$ & $2.1100 e+000$ & $7.8070 e+004$ \\
\hline$Y-90$ & $3.4995 e+000$ & $1.2948 \mathrm{e}+011$ & $8.7400 \mathrm{e}+003$ & $3.2338 \mathrm{e}+008$ \\
\hline \multicolumn{5}{|c|}{$\begin{array}{l}\text { Buildup: The material reference is Source } \\
\text { Integration Parameters }\end{array}$} \\
\hline \multicolumn{4}{|c|}{ Radial } & 20 \\
\hline \multicolumn{4}{|c|}{ Circumferential } & 20 \\
\hline
\end{tabular}




\begin{tabular}{|c|c|c|c|c|c|}
\hline \multicolumn{5}{|c|}{ Y Direction (axial) } & 20 \\
\hline \multicolumn{6}{|c|}{ Results - Dose Point \# 1 - $(38.5826,1.1,0) \mathrm{cm}$} \\
\hline Energy (MeV) & Activity (Photons/sec) & $\begin{array}{c}\text { Fluence Rate } \\
\mathrm{MeV} / \mathrm{cm}^{2} / \mathrm{sec} \\
\text { No Buildup }\end{array}$ & $\begin{array}{l}\text { Fluence Rate } \\
\mathrm{MeV} / \mathrm{cm}^{2} / \mathrm{sec} \\
\text { With Buildup }\end{array}$ & $\begin{array}{c}\text { Exposure Rate } \\
\mathrm{mR} / \mathrm{hr} \\
\text { No Buildup }\end{array}$ & $\begin{array}{c}\text { Exposure Rate } \\
\mathrm{mR} / \mathrm{hr} \\
\text { With Buildup }\end{array}$ \\
\hline 0.0419 & $1.120 \mathrm{e}+10$ & $1.357 e+03$ & $2.024 \mathrm{e}+03$ & $5.352 e+00$ & $7.979 \mathrm{e}+00$ \\
\hline 0.5875 & $8.505 e+07$ & $8.664 \mathrm{e}+02$ & $1.780 \mathrm{e}+03$ & $1.693 e+00$ & $3.479 \mathrm{e}+00$ \\
\hline 0.6617 & $9.861 \mathrm{e}+10$ & $1.181 \mathrm{e}+06$ & $2.332 \mathrm{e}+06$ & $2.290 \mathrm{e}+03$ & $4.521 \mathrm{e}+03$ \\
\hline 0.7873 & $6.758 \mathrm{e}+07$ & $1.026 e+03$ & $1.917 \mathrm{e}+03$ & $1.956 \mathrm{e}+00$ & $3.653 e+00$ \\
\hline 0.8759 & $6.383 e+07$ & $1.121 \mathrm{e}+03$ & $2.027 e+03$ & $2.108 \mathrm{e}+00$ & $3.812 \mathrm{e}+00$ \\
\hline 1.0017 & $1.407 \mathrm{e}+08$ & $2.966 \mathrm{e}+03$ & $5.155 e+03$ & $5.466 e+00$ & $9.500 \mathrm{e}+00$ \\
\hline 1.0441 & $1.131 \mathrm{e}+06$ & $2.523 e+01$ & $4.331 \mathrm{e}+01$ & $4.615 \mathrm{e}-02$ & $7.923 e-02$ \\
\hline 1.1185 & $5.139 e+05$ & $1.259 \mathrm{e}+01$ & $2.118 \mathrm{e}+01$ & $2.272 \mathrm{e}-02$ & $3.823 e-02$ \\
\hline 1.1421 & $3.170 \mathrm{e}+06$ & $7.988 \mathrm{e}+01$ & $1.336 \mathrm{e}+02$ & $1.436 \mathrm{e}-01$ & $2.401 \mathrm{e}-01$ \\
\hline 1.1732 & $1.150 \mathrm{e}+07$ & $3.004 e+02$ & $4.985 e+02$ & $5.369 \mathrm{e}-01$ & $8.908 \mathrm{e}-01$ \\
\hline 1.2456 & $5.139 e+06$ & $1.457 \mathrm{e}+02$ & $2.376 \mathrm{e}+02$ & $2.568 \mathrm{e}-01$ & $4.189 \mathrm{e}-01$ \\
\hline 1.2745 & $1.772 \mathrm{e}+08$ & $5.181 \mathrm{e}+03$ & $8.396 e+03$ & $9.086 e+00$ & $1.472 \mathrm{e}+01$ \\
\hline 1.3325 & $1.150 \mathrm{e}+07$ & $3.569 \mathrm{e}+02$ & $5.714 \mathrm{e}+02$ & $6.193 e-01$ & $9.913 e-01$ \\
\hline 1.3652 & $1.284 \mathrm{e}+06$ & $4.118 \mathrm{e}+01$ & $6.548 \mathrm{e}+01$ & $7.101 \mathrm{e}-02$ & $1.129 \mathrm{e}-01$ \\
\hline 1.4944 & $3.243 e+06$ & $1.175 e+02$ & $1.825 \mathrm{e}+02$ & $1.978 \mathrm{e}-01$ & $3.073 e-01$ \\
\hline 1.5952 & $1.435 e+07$ & $5.673 e+02$ & $8.675 e+02$ & $9.385 e-01$ & $1.435 e+00$ \\
\hline Totals & $1.104 \mathrm{e}+11$ & $1.195 e+06$ & $2.356 e+06$ & $2.319 e+03$ & $4.568 \mathrm{e}+03$ \\
\hline \multicolumn{6}{|c|}{ Results - Dose Point \# 2 - $(69.0626,1.1,0) \mathrm{cm}$} \\
\hline Energy (MeV) & Activity (Photons/sec) & $\begin{array}{c}\text { Fluence Rate } \\
\mathrm{MeV} / \mathrm{cm}^{2} / \mathrm{sec} \\
\text { No Buildup }\end{array}$ & $\begin{array}{l}\text { Fluence Rate } \\
\text { MeV/cm²/sec } \\
\text { With Buildup }\end{array}$ & $\begin{array}{c}\text { Exposure Rate } \\
\mathrm{mR} / \mathrm{hr} \\
\text { No Buildup } \\
\end{array}$ & $\begin{array}{c}\text { Exposure Rate } \\
\mathrm{mR} / \mathrm{hr} \\
\text { With Buildup }\end{array}$ \\
\hline 0.0419 & $1.120 \mathrm{e}+10$ & $3.862 \mathrm{e}+02$ & $5.782 \mathrm{e}+02$ & $1.523 e+00$ & $2.280 \mathrm{e}+00$ \\
\hline 0.5875 & $8.505 e+07$ & $2.572 \mathrm{e}+02$ & $5.387 e+02$ & $5.026 e-01$ & $1.053 e+00$ \\
\hline 0.6617 & $9.861 \mathrm{e}+10$ & $3.512 \mathrm{e}+05$ & $7.061 \mathrm{e}+05$ & $6.808 \mathrm{e}+02$ & $1.369 \mathrm{e}+03$ \\
\hline 0.7873 & $6.758 \mathrm{e}+07$ & $3.057 e+02$ & $5.809 e+02$ & $5.825 e-01$ & $1.107 e+00$ \\
\hline 0.8759 & $6.383 e+07$ & $3.343 e+02$ & $6.145 e+02$ & $6.286 e-01$ & $1.156 \mathrm{e}+00$ \\
\hline 1.0017 & $1.407 e+08$ & $8.861 \mathrm{e}+02$ & $1.564 \mathrm{e}+03$ & $1.633 e+00$ & $2.882 e+00$ \\
\hline 1.0441 & $1.131 \mathrm{e}+06$ & $7.540 e+00$ & $1.314 \mathrm{e}+01$ & $1.379 \mathrm{e}-02$ & $2.404 \mathrm{e}-02$ \\
\hline 1.1185 & $5.139 e+05$ & $3.765 e+00$ & $6.429 \mathrm{e}+00$ & $6.797 e-03$ & $1.160 \mathrm{e}-02$ \\
\hline 1.1421 & $3.170 \mathrm{e}+06$ & $2.390 \mathrm{e}+01$ & $4.055 e+01$ & $4.296 e-02$ & $7.288 \mathrm{e}-02$ \\
\hline 1.1732 & $1.150 \mathrm{e}+07$ & $8.992 e+01$ & $1.514 \mathrm{e}+02$ & $1.607 e-01$ & $2.705 \mathrm{e}-01$ \\
\hline 1.2456 & $5.139 \mathrm{e}+06$ & $4.362 e+01$ & $7.215 e+01$ & $7.692 e-02$ & $1.272 \mathrm{e}-01$ \\
\hline 1.2745 & $1.772 \mathrm{e}+08$ & $1.552 e+03$ & $2.550 \mathrm{e}+03$ & $2.722 \mathrm{e}+00$ & $4.472 e+00$ \\
\hline 1.3325 & $1.150 \mathrm{e}+07$ & $1.070 \mathrm{e}+02$ & $1.736 \mathrm{e}+02$ & $1.856 \mathrm{e}-01$ & $3.012 \mathrm{e}-01$ \\
\hline 1.3652 & $1.284 \mathrm{e}+06$ & $1.234 \mathrm{e}+01$ & $1.990 \mathrm{e}+01$ & $2.129 e-02$ & $3.431 \mathrm{e}-02$ \\
\hline 1.4944 & $3.243 e+06$ & $3.525 e+01$ & $5.547 e+01$ & $5.937 e-02$ & $9.342 \mathrm{e}-02$ \\
\hline 1.5952 & $1.435 e+07$ & $1.704 e+02$ & $2.638 \mathrm{e}+02$ & $2.818 \mathrm{e}-01$ & $4.363 e-01$ \\
\hline Totals & $1.104 \mathrm{e}+11$ & $3.554 \mathrm{e}+05$ & $7.133 e+05$ & $6.892 e+02$ & $1.383 e+03$ \\
\hline \multicolumn{6}{|c|}{ Results - Dose Point \# 3 - $(99.5426,1.1,0) \mathrm{cm}$} \\
\hline Energy (MeV) & Activity (Photons/sec) & $\begin{array}{c}\text { Fluence Rate } \\
\text { MeV/ } \mathrm{cm}^{2} / \mathrm{sec} \\
\text { No Buildup }\end{array}$ & $\begin{array}{l}\text { Fluence Rate } \\
\text { MeV/cm²/sec } \\
\text { With Buildup }\end{array}$ & $\begin{array}{c}\text { Exposure Rate } \\
\mathrm{mR} / \mathrm{hr} \\
\text { No Buildup }\end{array}$ & $\begin{array}{c}\text { Exposure Rate } \\
\mathrm{mR} / \mathrm{hr} \\
\text { With Buildup }\end{array}$ \\
\hline 0.0419 & $1.120 \mathrm{e}+10$ & $1.792 e+02$ & $2.692 e+02$ & $7.066 \mathrm{e}-01$ & $1.062 e+00$ \\
\hline 0.5875 & $8.505 e+07$ & $1.215 \mathrm{e}+02$ & $2.566 \mathrm{e}+02$ & $2.374 \mathrm{e}-01$ & $5.014 \mathrm{e}-01$ \\
\hline 0.6617 & $9.861 \mathrm{e}+10$ & $1.660 \mathrm{e}+05$ & $3.364 e+05$ & $3.218 \mathrm{e}+02$ & $6.522 e+02$ \\
\hline 0.7873 & $6.758 \mathrm{e}+07$ & $1.446 e+02$ & $2.769 \mathrm{e}+02$ & $2.756 \mathrm{e}-01$ & $5.276 \mathrm{e}-01$ \\
\hline 0.8759 & $6.383 e+07$ & $1.582 e+02$ & $2.930 \mathrm{e}+02$ & $2.976 \mathrm{e}-01$ & $5.510 e-01$ \\
\hline 1.0017 & $1.407 e+08$ & $4.197 e+02$ & $7.458 \mathrm{e}+02$ & $7.735 e-01$ & $1.374 \mathrm{e}+00$ \\
\hline 1.0441 & $1.131 \mathrm{e}+06$ & $3.573 e+00$ & $6.268 \mathrm{e}+00$ & $6.535 e-03$ & $1.147 \mathrm{e}-02$ \\
\hline 1.1185 & $5.139 e+05$ & $1.785 e+00$ & $3.066 \mathrm{e}+00$ & $3.221 \mathrm{e}-03$ & $5.535 e-03$ \\
\hline 1.1421 & $3.170 \mathrm{e}+06$ & $1.133 e+01$ & $1.934 \mathrm{e}+01$ & $2.036 \mathrm{e}-02$ & $3.476 \mathrm{e}-02$ \\
\hline 1.1732 & $1.150 \mathrm{e}+07$ & $4.263 e+01$ & $7.220 \mathrm{e}+01$ & $7.618 \mathrm{e}-02$ & $1.290 \mathrm{e}-01$ \\
\hline
\end{tabular}




\begin{tabular}{|c|c|c|c|c|c|}
\hline 1.2456 & $5.139 \mathrm{e}+06$ & $2.069 \mathrm{e}+01$ & $3.442 \mathrm{e}+01$ & $3.648 \mathrm{e}-02$ & $6.069 \mathrm{e}-02$ \\
\hline 1.2745 & $1.772 \mathrm{e}+08$ & $7.361 \mathrm{e}+02$ & $1.217 \mathrm{e}+03$ & $1.291 \mathrm{e}+00$ & $2.134 \mathrm{e}+00$ \\
\hline 1.3325 & $1.150 \mathrm{e}+07$ & $5.075 e+01$ & $8.282 e+01$ & $8.805 \mathrm{e}-02$ & $1.437 \mathrm{e}-01$ \\
\hline 1.3652 & $1.284 \mathrm{e}+06$ & $5.857 e+00$ & $9.493 e+00$ & $1.010 \mathrm{e}-02$ & $1.637 \mathrm{e}-02$ \\
\hline 1.4944 & $3.243 e+06$ & $1.673 e+01$ & $2.647 e+01$ & $2.818 \mathrm{e}-02$ & $4.458 \mathrm{e}-02$ \\
\hline 1.5952 & $1.435 \mathrm{e}+07$ & $8.090 \mathrm{e}+01$ & $1.259 \mathrm{e}+02$ & $1.338 \mathrm{e}-01$ & $2.082 \mathrm{e}-01$ \\
\hline Totals & $1.104 \mathrm{e}+11$ & $1.680 \mathrm{e}+05$ & $3.398 \mathrm{e}+05$ & $3.258 \mathrm{e}+02$ & $6.590 \mathrm{e}+02$ \\
\hline \multicolumn{6}{|c|}{ Results - Dose Point \# 4 - $(160.5026,1.1,0) \mathrm{cm}$} \\
\hline Energy (MeV) & Activity (Photons/sec) & $\begin{array}{l}\text { Fluence Rate } \\
\mathrm{MeV} / \mathrm{cm}^{2} / \mathrm{sec} \\
\text { No Buildup }\end{array}$ & $\begin{array}{l}\text { Fluence Rate } \\
\mathrm{MeV} / \mathrm{cm}^{2} / \mathrm{sec} \\
\text { With Buildup }\end{array}$ & $\begin{array}{c}\text { Exposure Rate } \\
\mathrm{mR} / \mathrm{hr} \\
\text { No Buildup }\end{array}$ & $\begin{array}{c}\text { Exposure Rate } \\
\mathrm{mR} / \mathrm{hr} \\
\text { With Buildup }\end{array}$ \\
\hline 0.0419 & $1.120 \mathrm{e}+10$ & $6.631 \mathrm{e}+01$ & $1.002 \mathrm{e}+02$ & $2.615 \mathrm{e}-01$ & $3.950 \mathrm{e}-01$ \\
\hline 0.5875 & $8.505 e+07$ & $4.588 \mathrm{e}+01$ & $9.778 \mathrm{e}+01$ & $8.965 e-02$ & $1.911 \mathrm{e}-01$ \\
\hline 0.6617 & $9.861 \mathrm{e}+10$ & $6.271 \mathrm{e}+04$ & $1.282 \mathrm{e}+05$ & $1.216 \mathrm{e}+02$ & $2.486 \mathrm{e}+02$ \\
\hline 0.7873 & $6.758 \mathrm{e}+07$ & $5.469 e+01$ & $1.056 \mathrm{e}+02$ & $1.042 \mathrm{e}-01$ & $2.012 \mathrm{e}-01$ \\
\hline 0.8759 & $6.383 e+07$ & $5.988 \mathrm{e}+01$ & $1.117 \mathrm{e}+02$ & $1.126 \mathrm{e}-01$ & $2.101 \mathrm{e}-01$ \\
\hline 1.0017 & $1.407 e+08$ & $1.590 \mathrm{e}+02$ & $2.845 e+02$ & $2.929 \mathrm{e}-01$ & $5.243 e-01$ \\
\hline 1.0441 & $1.131 \mathrm{e}+06$ & $1.353 e+00$ & $2.391 \mathrm{e}+00$ & $2.476 \mathrm{e}-03$ & $4.374 \mathrm{e}-03$ \\
\hline 1.1185 & $5.139 e+05$ & $6.763 e-01$ & $1.170 \mathrm{e}+00$ & $1.221 \mathrm{e}-03$ & $2.112 \mathrm{e}-03$ \\
\hline 1.1421 & $3.170 \mathrm{e}+06$ & $4.294 e+00$ & $7.381 \mathrm{e}+00$ & $7.717 e-03$ & $1.327 e-02$ \\
\hline 1.1732 & $1.150 \mathrm{e}+07$ & $1.616 \mathrm{e}+01$ & $2.755 e+01$ & $2.888 \mathrm{e}-02$ & $4.923 e-02$ \\
\hline 1.2456 & $5.139 e+06$ & $7.844 e+00$ & $1.314 \mathrm{e}+01$ & $1.383 e-02$ & $2.316 \mathrm{e}-02$ \\
\hline 1.2745 & $1.772 \mathrm{e}+08$ & $2.792 e+02$ & $4.644 \mathrm{e}+02$ & $4.896 \mathrm{e}-01$ & $8.144 \mathrm{e}-01$ \\
\hline 1.3325 & $1.150 \mathrm{e}+07$ & $1.925 \mathrm{e}+01$ & $3.161 \mathrm{e}+01$ & $3.340 e-02$ & $5.485 e-02$ \\
\hline 1.3652 & $1.284 \mathrm{e}+06$ & $2.222 e+00$ & $3.624 \mathrm{e}+00$ & $3.831 \mathrm{e}-03$ & $6.249 \mathrm{e}-03$ \\
\hline 1.4944 & $3.243 e+06$ & $6.351 \mathrm{e}+00$ & $1.011 \mathrm{e}+01$ & $1.070 \mathrm{e}-02$ & $1.702 \mathrm{e}-02$ \\
\hline 1.5952 & $1.435 \mathrm{e}+07$ & $3.072 \mathrm{e}+01$ & $4.807 e+01$ & $5.081 \mathrm{e}-02$ & $7.952 \mathrm{e}-02$ \\
\hline Totals & $1.104 \mathrm{e}+11$ & $6.347 e+04$ & $1.295 e+05$ & $1.231 \mathrm{e}+02$ & $2.512 \mathrm{e}+02$ \\
\hline \multicolumn{6}{|c|}{ Results - Dose Point \# 5 - $(251.9426,1.1,0) \mathrm{cm}$} \\
\hline Energy (MeV) & Activity (Photons/sec) & $\begin{array}{l}\text { Fluence Rate } \\
\mathrm{MeV} / \mathrm{cm}^{2} / \mathrm{sec} \\
\text { No Buildup }\end{array}$ & $\begin{array}{l}\text { Fluence Rate } \\
\mathrm{MeV} / \mathrm{cm}^{2} / \mathrm{sec} \\
\text { With Buildup }\end{array}$ & $\begin{array}{c}\text { Exposure Rate } \\
\mathrm{mR} / \mathrm{hr} \\
\text { No Buildup }\end{array}$ & $\begin{array}{c}\text { Exposure Rate } \\
\mathrm{mR} / \mathrm{hr} \\
\text { With Buildup }\end{array}$ \\
\hline 0.0419 & $1.120 \mathrm{e}+10$ & $2.594 \mathrm{e}+01$ & $3.946 \mathrm{e}+01$ & $1.023 \mathrm{e}-01$ & $1.556 \mathrm{e}-01$ \\
\hline 0.5875 & $8.505 e+07$ & $1.832 \mathrm{e}+01$ & $3.940 \mathrm{e}+01$ & $3.580 \mathrm{e}-02$ & $7.700 \mathrm{e}-02$ \\
\hline 0.6617 & $9.861 \mathrm{e}+10$ & $2.506 e+04$ & $5.168 \mathrm{e}+04$ & $4.859 \mathrm{e}+01$ & $1.002 \mathrm{e}+02$ \\
\hline 0.7873 & $6.758 \mathrm{e}+07$ & $2.188 \mathrm{e}+01$ & $4.256 \mathrm{e}+01$ & $4.169 \mathrm{e}-02$ & $8.110 \mathrm{e}-02$ \\
\hline 0.8759 & $6.383 e+07$ & $2.397 e+01$ & $4.505 e+01$ & $4.507 e-02$ & $8.472 \mathrm{e}-02$ \\
\hline 1.0017 & $1.407 e+08$ & $6.368 \mathrm{e}+01$ & $1.147 \mathrm{e}+02$ & $1.173 e-01$ & $2.115 \mathrm{e}-01$ \\
\hline 1.0441 & $1.131 \mathrm{e}+06$ & $5.422 \mathrm{e}-01$ & $9.645 \mathrm{e}-01$ & $9.919 \mathrm{e}-04$ & $1.764 \mathrm{e}-03$ \\
\hline 1.1185 & $5.139 \mathrm{e}+05$ & $2.711 \mathrm{e}-01$ & $4.720 \mathrm{e}-01$ & $4.893 e-04$ & $8.519 \mathrm{e}-04$ \\
\hline 1.1421 & $3.170 \mathrm{e}+06$ & $1.721 \mathrm{e}+00$ & $2.977 \mathrm{e}+00$ & $3.093 \mathrm{e}-03$ & $5.351 \mathrm{e}-03$ \\
\hline 1.1732 & $1.150 \mathrm{e}+07$ & $6.478 \mathrm{e}+00$ & $1.111 \mathrm{e}+01$ & $1.158 \mathrm{e}-02$ & $1.986 \mathrm{e}-02$ \\
\hline 1.2456 & $5.139 \mathrm{e}+06$ & $3.146 \mathrm{e}+00$ & $5.300 \mathrm{e}+00$ & $5.547 e-03$ & $9.345 e-03$ \\
\hline 1.2745 & $1.772 e+08$ & $1.120 \mathrm{e}+02$ & $1.874 e+02$ & $1.964 \mathrm{e}-01$ & $3.286 \mathrm{e}-01$ \\
\hline 1.3325 & $1.150 e+07$ & $7.723 e+00$ & $1.276 e+01$ & $1.340 \mathrm{e}-02$ & $2.213 e-02$ \\
\hline 1.3652 & $1.284 e+06$ & $8.915 e-01$ & $1.462 \mathrm{e}+00$ & $1.537 \mathrm{e}-03$ & $2.522 \mathrm{e}-03$ \\
\hline 1.4944 & $3.243 e+06$ & $2.549 e+00$ & $4.079 e+00$ & $4.294 \mathrm{e}-03$ & $6.869 \mathrm{e}-03$ \\
\hline 1.5952 & $1.435 e+07$ & $1.233 e+01$ & $1.940 \mathrm{e}+01$ & $2.040 \mathrm{e}-02$ & $3.209 \mathrm{e}-02$ \\
\hline Totals & $1.104 \mathrm{e}+11$ & $2.536 \mathrm{e}+04$ & $5.221 \mathrm{e}+04$ & $4.919 e+01$ & $1.012 \mathrm{e}+02$ \\
\hline \multicolumn{6}{|c|}{ Results - Dose Point \# 6 - $(312.9026,1.1,0) \mathrm{cm}$} \\
\hline Energy (MeV) & Activity (Photons/sec) & $\begin{array}{c}\text { Fluence Rate } \\
\mathrm{MeV} / \mathrm{cm}^{2} / \mathrm{sec} \\
\text { No Buildup }\end{array}$ & $\begin{array}{l}\text { Fluence Rate } \\
\mathrm{MeV} / \mathrm{cm}^{2} / \mathrm{sec} \\
\text { With Buildup }\end{array}$ & $\begin{array}{c}\text { Exposure Rate } \\
\mathrm{mR} / \mathrm{hr} \\
\text { No Buildup }\end{array}$ & $\begin{array}{c}\text { Exposure Rate } \\
\mathrm{mR} / \mathrm{hr} \\
\text { With Buildup }\end{array}$ \\
\hline 0.0419 & $1.120 \mathrm{e}+10$ & $1.647 \mathrm{e}+01$ & $2.517 \mathrm{e}+01$ & $6.496 \mathrm{e}-02$ & $9.925 \mathrm{e}-02$ \\
\hline 0.5875 & $8.505 e+07$ & $1.178 \mathrm{e}+01$ & $2.546 \mathrm{e}+01$ & $2.302 \mathrm{e}-02$ & $4.974 \mathrm{e}-02$ \\
\hline 0.6617 & $9.861 \mathrm{e}+10$ & $1.612 \mathrm{e}+04$ & $3.339 e+04$ & $3.125 \mathrm{e}+01$ & $6.473 e+01$ \\
\hline
\end{tabular}


KBC-40179 rev 1

\begin{tabular}{|l|l|l|l|l|l|}
\hline 0.7873 & $6.758 \mathrm{e}+07$ & $1.408 \mathrm{e}+01$ & $2.750 \mathrm{e}+01$ & $2.683 \mathrm{e}-02$ & $5.241 \mathrm{e}-02$ \\
\hline 0.8759 & $6.383 \mathrm{e}+07$ & $1.543 \mathrm{e}+01$ & $2.912 \mathrm{e}+01$ & $2.901 \mathrm{e}-02$ & $5.476 \mathrm{e}-02$ \\
\hline 1.0017 & $1.407 \mathrm{e}+08$ & $4.101 \mathrm{e}+01$ & $7.417 \mathrm{e}+01$ & $7.556 \mathrm{e}-02$ & $1.367 \mathrm{e}-01$ \\
\hline 1.0441 & $1.131 \mathrm{e}+06$ & $3.492 \mathrm{e}-01$ & $6.234 \mathrm{e}-01$ & $6.388 \mathrm{e}-04$ & $1.140 \mathrm{e}-03$ \\
\hline 1.1185 & $5.139 \mathrm{e}+05$ & $1.746 \mathrm{e}-01$ & $3.051 \mathrm{e}-01$ & $3.152 \mathrm{e}-04$ & $5.507 \mathrm{e}-04$ \\
\hline 1.1421 & $3.170 \mathrm{e}+06$ & $1.109 \mathrm{e}+00$ & $1.925 \mathrm{e}+00$ & $1.993 \mathrm{e}-03$ & $3.459 \mathrm{e}-03$ \\
\hline 1.1732 & $1.150 \mathrm{e}+07$ & $4.174 \mathrm{e}+00$ & $7.185 \mathrm{e}+00$ & $7.459 \mathrm{e}-03$ & $1.284 \mathrm{e}-02$ \\
\hline 1.2456 & $5.139 \mathrm{e}+06$ & $2.027 \mathrm{e}+00$ & $3.427 \mathrm{e}+00$ & $3.574 \mathrm{e}-03$ & $6.042 \mathrm{e}-03$ \\
\hline 1.2745 & $1.772 \mathrm{e}+08$ & $7.215 \mathrm{e}+01$ & $1.211 \mathrm{e}+02$ & $1.265 \mathrm{e}-01$ & $2.124 \mathrm{e}-01$ \\
\hline 1.3325 & $1.150 \mathrm{e}+07$ & $4.977 \mathrm{e}+00$ & $8.247 \mathrm{e}+00$ & $8.636 \mathrm{e}-03$ & $1.431 \mathrm{e}-02$ \\
\hline 1.3652 & $1.284 \mathrm{e}+06$ & $5.746 \mathrm{e}-01$ & $9.454 \mathrm{e}-01$ & $9.909 \mathrm{e}-04$ & $1.630 \mathrm{e}-03$ \\
\hline 1.4944 & $3.243 \mathrm{e}+06$ & $1.644 \mathrm{e}+00$ & $2.637 \mathrm{e}+00$ & $2.768 \mathrm{e}-03$ & $4.441 \mathrm{e}-03$ \\
\hline 1.5952 & $1.435 \mathrm{e}+07$ & $7.953 \mathrm{e}+00$ & $1.255 \mathrm{e}+01$ & $1.316 \mathrm{e}-02$ & $2.075 \mathrm{e}-02$ \\
\hline Totals & $1.104 \mathrm{e}+11$ & $1.631 \mathrm{e}+04$ & $3.373 \mathrm{e}+04$ & $3.163 \mathrm{e}+01$ & $6.540 \mathrm{e}+01$ \\
\hline
\end{tabular}

